

क्่ Rutherford Appleton Laboratory

这 Chilton DIDCOT Oxon OX11 0QX

RAL-92-083

\title{
Photon Scattering by Magnetic Solids
}

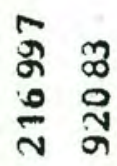

宓

S W Lovesey

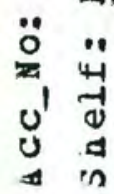

December 1992

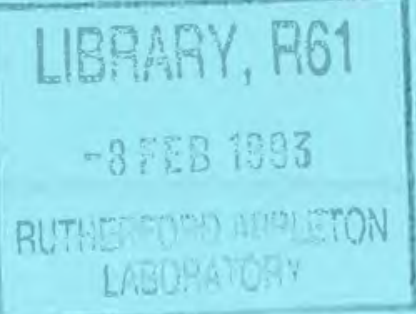


Science and Engineering Research Council

"The Science and Engineering Research Council does not accept any responsibility for loss or damage arising from

the use of information contained in any of its reports or

in any communication about its tests or investigations" 


\title{
PHOTON SCATTERING BY MAGNETIC SOLIDS
}

\author{
Stephen W Lovesey \\ Rutherford Appleton Laboratory \\ Oxfordshire OX11 0QX
}

Article prepared for Reports on Progress in Physics 



\section{Contents}

1. Introduction

1.1 Orientation to Scattering by Magnetic Solids

1.2 Elements of Charge Scattering

2. Classical Description of the Scattering of High Frequency Radiation

3. Polarization Effects and Magnetic Scattering Amplitude

\subsection{Polarization States}

3.2 Density Matrix

\subsection{Scattering Amplitude}

4. Partial Differential Cross-Section

5. Correlation Functions

6. Spontaneous Emission

7. Scattering by Free Charges

8. Compton Limit

9. Generalized Kramers-Heisenberg Formula

10. Scattering by Orbital Magnetism

11. Scattering by Spin Magnetism

12. Diffraction

12.1 Survey

12.2 Pure Charge Scattering

12.3 Unpolarized Primary Beam 
12,4 Linear Polarization

12.5 Circular Polarization

13. Spectroscopy

13.1 Prologue

13.2 Cross-Section

13.3 Compton Limit

13.3.1 Charge Scattering

13.3.2 Orbital Contribution

\subsubsection{Spin Contribution}

14. Dichroism

14,1 Circular Dichroism

14.2 Linear Dichroism

15. Elastic Resonant Scattering

15.1 Resonant Scattering Amplitude

16. Concluding Remarks 


\section{List of Important Symbols}

A

$a_{0}$

c

E

$\mathrm{E}, \mathrm{E}^{\prime}$

e

$\mathrm{F}_{\mathrm{c}}$

$\mathbf{F}_{\mathrm{s}}, \mathbf{F}_{l}$

G

g

H

$\mathscr{H}$

$\mathrm{k}_{\mathrm{B}}$

$\mathrm{J}(\mathrm{q})$

$k=q-q^{\prime}$

$\mathrm{m}$

m*

$N(k)$

$\mathrm{n}(\mathrm{k})$

p

$\mathrm{P}_{\mathrm{i}}$

q

$\mathbf{R}$

$r_{e}$

$R_{\infty}$

$\mathrm{S}(\mathrm{k})$ vector potential

Bohr radius

velocity of light

electric field

primary and secondary photon energies

unit of charge for an electron

change unit cell structure factor

unit cell structure factors for spin and orbital magnetism

scattering amplitude operator

gyromagnetic factor

magnetic field

Hamiltonian operator for electrons in a solid

Boltzmann constant

current density

scattering vector

mass of an electron

effective mass of a charge carrier

average particle density

Fourier transform of the single particle density function

linear momentum operator

Stokes paramelers, $\mathrm{i}=1,2,3$

wave vector of photon; $\mathbf{q}, \mathbf{q}^{\prime}$ wave vectors for primary and secondary photons

position vector

classical radius of an electron

Rydberg unit of energy

spin density operator 


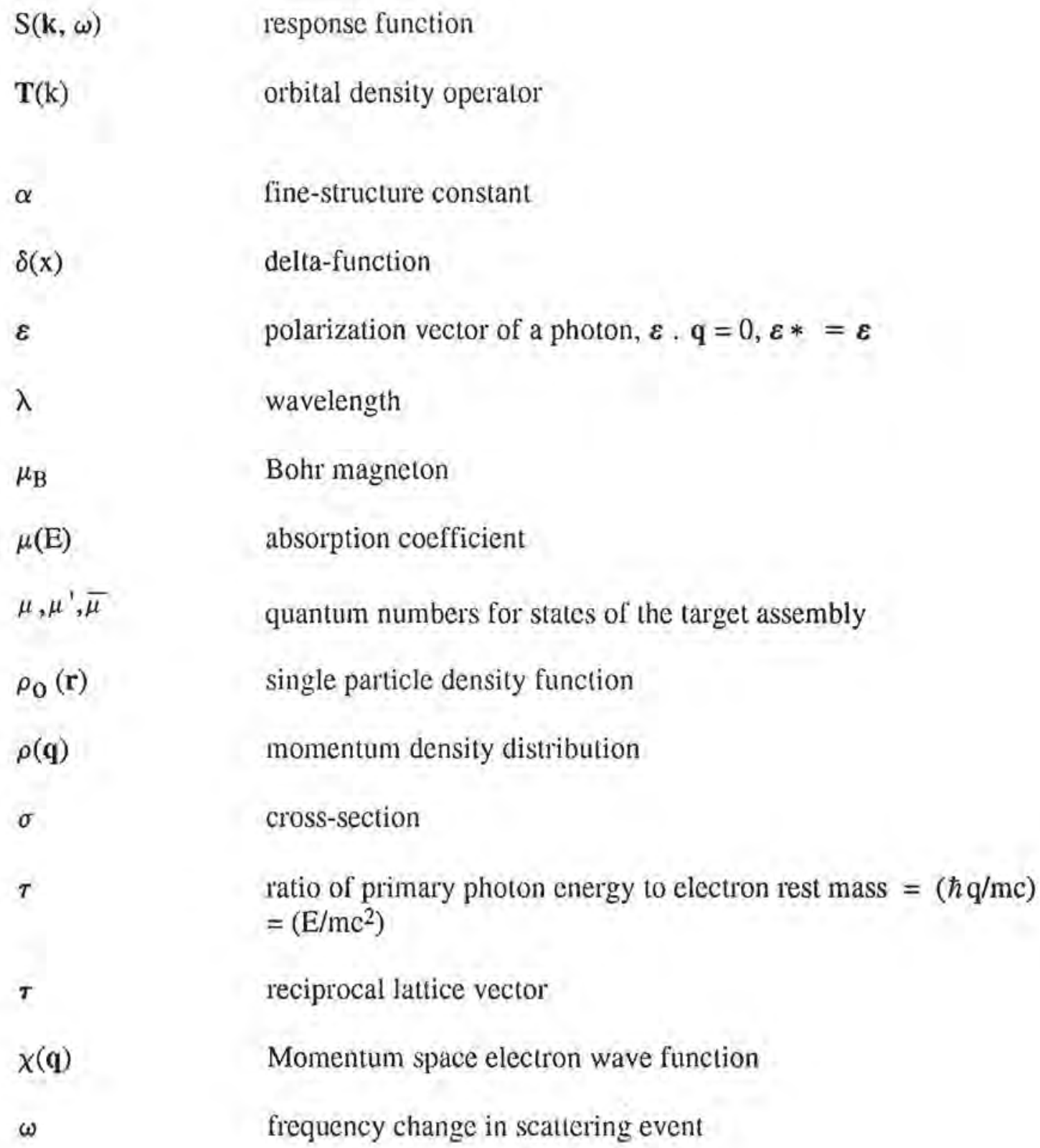




\begin{tabular}{|c|c|c|}
\hline Quantity & Symbol & Value \\
\hline Bohr radius & $a_{0}=\hbar^{2} / \mathrm{me}^{2}$ & $0.529 \AA$ \\
\hline Planck's constant $/ 2 \pi$ & $\hbar$ & $0.658 \mathrm{meV}$ psec \\
\hline Rydberg & $\mathrm{R}_{\infty}=1 / 2 m c^{2} \alpha^{2}=\mathrm{e}^{2} / 2 \mathrm{a}_{0}$ & $13.606 \mathrm{eV}$ \\
\hline Fine-structure constant & $\alpha=\mathrm{e}^{2} / \hbar \mathrm{c}$ & $1 / 137.04$ \\
\hline $\begin{array}{l}\text { Rest mass energy of the } \\
\text { electron }\end{array}$ & $\mathrm{mc}^{2}$ & $0.511 \mathrm{MeV}$ \\
\hline Bohr magneton & $\mu_{\mathrm{B}}=\mathrm{e} \hbar / 2 \mathrm{mc}$ & $0.579 \times 10^{-1} \mathrm{meV} \mathrm{T}^{-1}$ \\
\hline $\begin{array}{l}\text { Classical radius of the } \\
\text { electron }\end{array}$ & $r_{e}=e^{2 / m c^{2}}$ & $0.282 \times 10^{-12} \mathrm{~cm}$ \\
\hline & $\hbar^{2} / 2 \mathrm{~m}$ & $3.810 \mathrm{eV} \AA^{2}$ \\
\hline
\end{tabular}

\section{Energy Units}

$1 \mathrm{meV}=0.24 \mathrm{THz}=8.07 \mathrm{~cm}^{-1}=11.61 \mathrm{~K}$

\section{General Units}

magnetic field $\mathrm{T}$ (Tesla $)=10^{4}$ gauss

$$
\begin{aligned}
& \text { psec }=10^{-12} \mathrm{sec} \\
& \mathrm{MeV}=10^{6} \mathrm{eV}: \mathrm{meV}=10^{-3} \mathrm{eV} \\
& \AA=10^{-8} \mathrm{~cm}
\end{aligned}
$$




\section{INTRODUCTION}

In the past few years, there has been much new activity in the study of the magnetic properties of materials using photon beams. Observable effects attributable to magnetic features of the sample are usually relatively small compared with charge induced effects, e.g. Thomson scattering. To a large extent, the new activity is an outcome of the advent of improved photon sources, based on electron synchrotrons, and improved photon optical elements (Margaritondo 1988, Gerson et al. 1992). Improvements over conventional laboratory $x$-ray generators include, a high brightness with the option of superior resolution, a high degree of linear polarization, tuneability of the primary photon energy, and the provision of good beams of circularly polarized photons. Recent progress in the study of magnetic materials is a beneficiary of earlier pioneering work by de Bergevin and Brunel; for a review, see de Bergevin and Brunel (1986).

The present review aims to gather basic physical ideas and theoretical concepts, and illustrate recent progress in using photon beams to study magnetic properties of materials, All being well, the material will be useful for the growing band of new researchers attracted to the subject by its scientific potential, and greater number of advanced photon sources. There is no attempt to address experimental issues which arise in either instrument design or data correction and analysis. With regard to theoretical work, technical aspects of computations, such as electronic band structure calculations and Hartree-Fock atomic calculations, are not reviewed. The degree of working provided here takes account of what is already readily available in review articles and books.

\subsection{Orientation to Scattering by Magnetic Solids}

By way of an orientation to the subject of magnetic photon scattering there is something to be gained in discussing the well-researched problem of the anomalous magnetic moment of the electron. In terms of the gyromagnetic factor ge, the current best value is

$$
\mathrm{g}_{\mathrm{e}}=2.0\{1.001159652193\}
$$

with an error of $10^{-11}$. The significance of this result in the present context is that the anomalous component, or correction to the naîve value 2.0 , can be viewed as arising from the interaction of the magnetic moment of an electron with the associated photon cloud (the physical clectron can emit or absorb a virtual photon-classically the electromagnetic field due to the presence of the electron can react on the electron itself - which produces a radiative correction to the magnetic moment, among other 
observable quantities). If the photon cloud is described by a vector potential $\mathbf{A}$, the corresponding magnetic field is proportional to curl $\mathbf{A}$, and the lowest-order approximation to the interaction that generates the anomalous component in $\mathrm{ge}_{\mathrm{e}}$ is s. curl A where s is the spin operator for the electron, i,e. the Zeeman interaction for a magnetic field $\propto$ curl $\mathbf{A}$. Hence, the first observation we make is that the anomalous component is an example of a magnetic interaction between photons and an electron. Just the same interaction will contribute to the scattering of a photon beam by magnetic electrons in a target sample, e.g. a solid with unpaired electrons. In a better approximation, the electron-photon interaction contains a term which can be interpreted as arising from the spin-orbit interaction. The latter is quadratic in $\mathbf{A}$, and in the calculation of the photon scattering amplitude it is added to the $\mathrm{A}^{2}$ term which generates (charge) Thomson scattering, whereas terms linear in A produce scattering in second-order processes and the charge terms generate the familiar Raman and resonant fluorescence effects. For target samples with unpaired electrons, the same second-order charge processes produce scattering from the orbital magnetic moments, as demonstrated first by deBergevin and Brunel (1981).

The anomalous magnetic moment of the electron serves to illustrate two other important issues in magnetic photon scattering. The anomalous component of $g_{e}$ is very small, so we are led to expect that the magnetic scattering amplitude is small compared to the corresponding charge amplitude. Indeed, the magnetic amplitude calculated by perturbation theory is smaller than the charge amplitude by a factor $\tau=$ (primary photon energy/mc $\mathrm{mc}^{2}$ ) where $m \mathrm{c}^{2}$ is the rest mass of the electron, i.e. $\tau \leq 0.1$ in many experiments. In addition to this small factor, it is to be noted that all electrons contribute to charge scattering, whereas it is just the small fraction of unpaired electrons which contribute to magnetic scattering. For example, $\mathrm{Z}$ electrons contribute from each atom to charge coherent scattering, and the few unpaired electrons in the $4 f$ shell of a rarc earth ion, say, contribute to Bragg magnetic scattering.

The last issue concerning $g_{e}$ that merits note at this juncture is that the measured anomalous component is in complete agreement with the theoretical prediction. Calculations are made using covariant perturbation theory within quantum electrodynamics (QED) and the degree of agreement between theory and experiment means that QED is a triumph of modern physics (Kinoshita 1990). The point to note in the present context is that the same theory, QED, underpins the theory of magnetic photon scattering by electrons in a condensed matter target (Platzman and Tzoar 1965, de Bergevin and Brunel 1981, Grotch et al. 1983). 


\subsection{Elements of Charge Scattering}

The interpretation of photon scattering (resonant and nonresonant events) from magnetic materials involves no really new ideas not encountered in photon scattering from unpolarized (paired) electrons. In view of this situation, it makes good sense to briefly review photon scattering from charge distributions in atoms and solids. The exercise will gather concepts used in remaining sections, where the emphasis is on magnetic scattering and resonant events, and affords an ideal opportunity to introduce basic notation. Events induced by a photon beam impinging on a solid are summarized in Fig (1). By and large, we do not derive results. Instead, we strive to use physical intuition and plausible arguments. Mathematical details can be found in several textbooks, e.g. Berestetskii et al. (1982), Newton (1982), Sakurai (1987) Weissbluth (1989) and Friedrich (1991).

We begin by considering the scattering of light by an atom. The scattering process consists of the absorption of a primary photon and the simultaneous emission of a secondary photon. The target atom may be left in either its initial state, in which case the scattering process is coherent (apart from incoherent processes that in origin are purely quantum mechanical), or, as in the Raman effect (Eteläniemi et al. 1992), in some other state.

The general character of the scattering process depends on whether the energy of the primary photon is of the same order of magnilude as the binding energy of the electron in the atom, or very large compared to the binding energy. In the latter case, the electron can be regarded as essentially free during the very brief duration of the photon-electron interaction. The energy required to remove one electron from a normal state to infinity (ionization energy) is $\mathrm{I} \sim 5-25 \mathrm{eV}$ on moving down the periodic table from $\mathrm{Lu}$ to $\mathrm{He}$. Of course, an important parameter in determining the character of the scattering process is the wavelength $\lambda$ of the photon relative to essential length scales, e.g. ionic radius or lattice spacing. For orientation it is useful to find the wavelength of a photon with an energy $=R_{\infty}=13.6 \mathrm{eV}$. In this case,

$$
(2 \pi \hbar c / \lambda)=R_{\infty}=e^{2} / 2 a_{0}
$$

or

$$
\lambda=4 \pi \mathrm{a}_{\mathrm{o}} / \alpha=911 \AA
$$

where $a_{o}$ and $\alpha$ are the Bohr radius and fine-structure constant, respectively. The calculation reveals that, a photon with an energy less than the ionization energy of 
normal atomic states has a wavelength two orders of magnitude larger than the size of a typical electron distribution of an ion, so soft $x$-rays are not suitable for the investigation of atomic charge distributions.

The conservation of energy requires that the energies of the secondary and primary photons differ by the energy difference of the atom. If the initial and final states of the atom have energies $E_{\mu}$ and $E_{\mu}$, respectively, and the wave vectors of the primary and secondary photons are $q, q^{\prime}$ then,

$$
\hbar c\left(q-q^{\prime}\right)=E_{\mu}-E_{\mu} .
$$

We assume for the moment that $\hbar \mathrm{cq}$ is not near a resonance frequency of the atom. In the case of coherent scattering the atom is left in the same state $\mu=\mu^{\prime}$. The wave vector of the secondary photon $q^{\prime}$ is then the same as that of the primary photon $q$. The case $E_{\mu} \neq E_{\mu}$ represents an inelastic scattering process, e.g. Raman or Compton processes. It is worth remarking that, Bragg scattering is coherent because the relevant mass in the kinematics of the process is the mass of the whole crystal, which is infinite to all intents and purposes. Even the recoil of an atom in a crystal destroys coherence in a scattering event.

The momentum of the electron-photon system will not in general be conserved in the interaction of a photon with an electron in a bound state of an atom or molecule, A way of accounting for the absence of momentum conservation is that, with the electron in a bound state the mass of the nucleus is involved in the kinematics of the scattering event, and this has a large capacity to take up momentum. On the other hand, the momentum will be conserved in the interaction of a photon with a free electron, an idealized event which nonetheless is useful to study in detail. It is found that momentum conservation has a significant effect on the features of the scattering cross-section.

The case of coherent scattcring is adequately described by a modest extension to the Kramers-Heisenberg dispersion formula published in 1925. The scattering of an electromagnetic wave can be visualized in classical mechanics by a straightforward two-step process, in which the elements are (i) a bound electron oscillates when exposed to a time-dependent electric field, and (ii) the oscillating charge in turn radiates an electromagnelic wave. If $\omega$ and $\omega_{o}$ are, respectively, the frequencies of the light and the bound electron, which is subject to a damping force of strength $\gamma$, the cross-section $\sigma$ for the total radiation scaltered is, 


$$
\sigma=\frac{8 \pi}{3} \mathrm{r}_{\mathrm{e}}^{2}\left\{\frac{\omega^{4}}{\left(\omega_{\mathrm{o}}^{2}-\omega^{2}\right)^{2}+(\gamma \omega)^{2}}\right\},
$$

where $r_{e} \sim 0.3 \times 10^{-12} \mathrm{~cm}$ is the classical radius of the electron. For $\omega_{o} \rightarrow 0$ and $\gamma \ll \omega$ we obtain the classical cross-section for scattering by a free electron,

$$
\sigma=\frac{8 \pi}{3} \mathrm{r}_{\mathrm{e}}^{2}=0.66 \times 10^{-24} \mathrm{~cm}^{2}
$$

Thus, the cross-section for scattering by a free electron is a universal constant and independent of the primary frequency. A complete treatment of this event leads to the celebrated formula for Compton scattering derived by Klein and Nishina in 1929 , and recorded in $\S 7$. When $\hbar \omega \ll m c^{2}$ this formula reduces to the classical nonrelativistic Thomson's formula egn (1.2).

For frequencies far away from the resonance frequency $(\gamma \omega)^{2}$ may be neglected in (1.1). On the other hand, in the neighbourhood of $\omega=\omega_{0}$ the crosssection becomes very large and we have the case of resonance fluorescence. In the denominator we may then put $\omega \sim \omega_{O}$, to arrive at the estimate,

$$
\sigma=\frac{2 \pi}{3}\left(\frac{r_{e}}{\omega_{0}}\right)^{2}\left\{\frac{\omega^{4}}{\left(\omega-\omega_{0}\right)^{2}+(\gamma / 2)^{2}}\right\} .
$$

The cross-section (1.3) has its maximum when $\omega=\omega_{o}$, and this value is, in order of magnitude, $\sigma_{\max } \sim\left(r_{e} \omega^{2} / \omega_{o} \gamma\right)^{2}$. A quantum mechanical calculation shows that the damping $\gamma \sim\left(\alpha \omega^{3} d^{2} / c^{2}\right)$ where $d$ is the oscillator strength for the transition in question. The Reiche - Thomas - Kuhn sum rule for oscillator strengths provides the estimate $d^{2} \sim\left(\hbar / \mathrm{m} \omega_{o}\right)$. Combining these results leads to $\sigma_{\max } \sim(\mathrm{c} / \omega)^{2} \sim \lambda^{2}$, i.e. the resonance cross-section is of the order of the square of the wavelength and independent of the fine structure constant, as compared with typical values $\sim r_{e}{ }^{2}$ outside the resonance region.

In the limit $\gamma=0$ and $\omega<\omega_{O}$ the cross-section (1.1) reduced to,

$$
\sigma=\frac{8 \pi}{3} r_{\mathrm{e}}^{2}\left(\omega / \omega_{\mathrm{o}}\right)^{4}
$$

Thus we see that the scattering cross-section at long wavelengths varies as the inverse of the fourth power of the wavelength; this observation is usually referred to a Rayleigh's law (1899). 
In the quantum theory of scaltering taken up in later parts of the article we shall meet formulae which are simple generalizations of (1.1), (1.3) and (1.4). Referring to Thomson's formula (1.2) note that $10^{-24} \mathrm{~cm}^{2}$ is a unit for cross-sections given the name of a barn.

When the Kramers-Heisenberg formula is derived using quantum mechanics, by making a straightforward application of time-dependent perturbation theory, it is quite naturally generalized to encompass inelastic scattering events. In this instance the atom does not remain in its initial state, and the energy of the primary and secondary photons are different. In atomic physics this phenomenon is called the Raman effect. From the formulation of the theory of the Raman effect by Placzek (1931-34) it is known to be described by modulation of the susceptibility (or, equivalently, polarizability) of the target assembly by the vibrations, and also the scattering by other excitations in solids, including plasmons, excitons and spinwaves, c.f. Hayes and Loudon (1978).

Let us now tum to questions about the intensity distribution of secondary photons. Rayleigh considered the problem of the scattering of light by $N$ particles with a separation much greater than the wavelength $\lambda$ of the light. In this instance, the particles scatter independently of each other, and the total cross-section is $N$ times the single particle cross-section (1.4).

For dense targets, the mean separation between particles is much less than $\lambda$, and there exists the possibility of geometric coherence in scattering from different particles which no longer act as independent scatterers. The foregoing case of Rayleigh scattering is an example of an incoherent scattering process. In scattering from a crystal (Bragg) peaks in the intensity distribution occur when the change in the wave vector of the radiation on scattering coincides with a crystal reciprocal lattice vector $\{\tau\}$, i.e. when Bragg's law is satisfied, namely,

$$
k=q-q^{\prime}=\tau .
$$

The scattering is strictly elastic, and of order $\mathrm{N}_{0}{ }^{2}$ where $\mathrm{N}_{0}$ is the number of scatterers. The cross-section is determined by the appropriate amplitude averaged over all parameters (disorder and quantum degeneracies) for otherwise it will not possess the full translational invariance of a perfect crystal structure.

Crystal defects, and other departures from a perfect crystal structure, produce diffuse scattering in the vicinity of Bragg peaks. A noncrystalline (homogeneous) target system can be regarded as possessing just one trivial reciprocal lattice vector $\tau=0$, i.e. the intensity in any direction other than forward is zero. However, spatial 
fluctuations in the density distribution produce scattering away from the forward direction.

To pursue the discussion of features of the intensity distribution of radiation scattered by an assembly of $N$ identical atoms confined to a region small compared with $\lambda$, it is helpful to express the cross-section in terms of matrix elements of a scattering amplitude operator. Let us denote the operator by G, and employ the notation $\left\langle\mu^{\prime}|G| \mu\right\rangle$ for the matrix element taken between initial and final states labelled by the quantum numbers $\mu, \mu^{\prime}$, respectively. The total cross-section is proportional to

$$
\left|\sum_{\mathrm{j}}\left\langle\mu^{\prime}\left|\mathrm{G}_{\mathrm{j}}\right| \mu\right\rangle\right|^{2},
$$

where the sum is over all $N$ atoms labelled by the index $j$. The wave functions used to calculate the matrix elements for several identical atoms taken together are not simply equal functions. The wave functions are essentially defined only to within an arbitrary phase factor, which is different for each atom. The cross-section has to be averaged over the phase factor of each atom separately.

The matrix element $\left\langle\mu^{\prime}|G| \mu\right\rangle$ of each atom includes a factor $e^{i\left(\phi-\phi^{\prime}\right)}$, where $\phi$ and $\phi^{\prime}$ are the phases of the wave functions of the initial and final states. For a Raman event, the states $\mid \mu>$ and $\mathrm{I} \mu^{\prime}>$ are different, and this factor is not equal to unity. In the squared modulus which appears in the cross-section, the products of terms pertaining to different atoms will include phase factors which vanish on independent averaging over the phases of the atoms, and only the squared modulus of each term remains. In consequence, the total cross-section for scattering by $N$ atoms is found by taking $N$ times the cross-section for scattering by one atom; the conclusion is that when $\mu \neq \mu^{\prime}$ scattering is incoherent.

But if $\mu=\mu^{\prime}$, then the factors $e^{i\left(\phi-\phi^{\prime}\right)}=1$. The amplitude for scattering by the assembly of atoms is $N$ times that for scattering by one atom, and the scattering crosssection consequently differs from a single atom by a factor $N^{2}$. This scattering is coherent, as has been mentioned previously. A feature not hitherto discussed is the effect of a degeneracy in the atomic state, e.g. a state characterized by a definite value of the total angular momentum oprator might exist in an environment which does not completely lift the degeneracy with respect to the magnetic quantum number. For such an energy level, there will also be incoherent scattering arising from the transitions of the atom between various mutually degenerate states. Note that this is a purely quantum effect, and in the classical theory any scattering without change of the frequency of the radiation is coherent. 
Finally, we shall discuss qualitatively the essential aspects of the cross-section as the energy of the primary photon increases to the stage when the wavelength becomes comparable with or smaller than the dimension of an atom. The key difference is that the plane-wave $\exp (i q$ q. $r)$ representing the photon is no longer a constant over the spatial region in which the atomic states in matrix elements have a significant amplitude, a region with dimension $\sim a_{o}$.

The numerical value of the matrix elements decreases when these exponential functions vary appreciably over a distance $\sim a_{o}$. Hence, the scattered intensity decreases for coherent events and inelastic events (at least when the atom is left in a discrete quantum state), Further increase of the energy of the primary photon leads to a vanishingly small cross-section. Such a situation is achieved when $\left(q a_{o}\right) \gg>1$, which can be expressed roughly by

$$
\hbar \mathrm{c} \mathrm{q} \gg(\mathrm{I} / \alpha \mathrm{Z})
$$

where $l$ is the ionization energy of the atom.

However, when this condition is well satisfied the electron can be promoted to a state in the continuum spectrum of the atom, and this provides an important contribution to the scaltered intensity which eventually dominates for extreme values of the primary photon energy. To appreciate how this comes about, take the final state to have a plane-wave character $\exp (i p . r)$. If $p \cong q-q^{\prime}$, the exponential factor in the matrix element $\exp \left\{i\left(q-q^{\prime}\right), r\right\}$ is just compensated by the plane-wave, and once more the matrix element becomes large, however small the primary wavelength may be. For a given angle of scattering, $q^{\prime}$ is determined entirely by the conservation of energy and $p \cong q-q^{\prime}$ which amounts to conservation of wave vector. Thus one observes an intense and narrow line in the spectrum, and since $\left(\mathrm{qa}_{\mathrm{o}}\right) \gg 1$ the scattering process is incoherent.

The physics behind the situation described is that, for very short wavelengths, the process considered becomes close to the ideal situation of scattering by a free electron; $\boldsymbol{p} \cong q-q^{\prime}$ expresses then the conservation of wave vector, which is always satisfied in the interaction with free electrons, since for $q \gg(I / \hbar c)$ the momentum of the electron in the initial bound state is relatively very small. The breadth of the narrow (Compton) line is deternined by the momentum in the initial bound state and it is often referred to as Doppler broadening. Complon, sometimes called deep inelastic (Taylor, 1991), scattering is a useful method for determining momentum distributions in atoms and solids.

To round off the present discussion, let us summarize the qualitative nature of the coherent and Compton scattering for increasing primary photon energy. At the 
two extremes $q<<(I / \hbar c)$ and $q>>(I / \hbar c)$ the spectrum is dominated by single components centred at $\omega=0$ and $\omega=$ free particle recoil energy, respectively, where $\hbar \omega$ is the energy transferred to the electrons. On moving between these extreme energies, the intensity is gradually transferred from one component to the other. The intermediate case, with significant intensity in both components, is achieved in scattering $\sim 50 \mathrm{keV}$ photons from light elements. The Compton intensity is sketched in Fig (2). The Doppler broadening is significant for core state electrons, whereas valence electrons are loosely bound and result in a relatively narrow peak. In Fig. (1) we summarize the main events induced by photons. In this article we are concerned only with scattering and resonant processes, and not the photoelectric effect or recoil electrons. 
Following the outline of the classical description of light scattering by an assembly of charges, let us consider in more detail the limit when the frequency of the primary radiation $c q$ is large compared with the fundamental internal frequencies of the atomic or molecular system. If the characteristic velocity and spatial dimension of the motion of the charges are $\mathrm{v}$ and $\mathrm{a}$, respectively, then $\omega_{o} \sim \mathrm{v} / a$, so the condition which is assumed to prevail is,

$$
c q \gg \omega_{o} \sim \mathrm{v} / a .
$$

For a classical description to be valid $\mathrm{v} \ll<c$, and in atomic systems $v \sim \alpha c$. Because of the relatively large mass of the nucleus, the scattering resulting from the nuclear charge can be safely neglected (the effective scattering length is $\sim r_{e} / 1836$ ).

According to the condition (2.1), the periods of the motion of the charges of the system are large compared with the period of the primary radiation. So during a time interval of the order of the period, the motion of the charges of the system can be considered uniform. This means that in considering the scattering of short wavelength radiation, it is not necessary to account for the interaction of the charges of the system with each other, that is, they can be considered as free particles in the relatively short duration of the photon-charge interaction. In calcuiating the velocity acquired by a charge in the field of the primary radiation, each charge in the system is considered separately. Furthermore, the scattering takes place without change in the state of the system. An alternative viewpoint is that the scattering event is essentially instantaneous, and consequently the charges appear as free particles while the system is too slow to change from the initial state.

The scattering process is therefore elastic, i.e. $\omega=0$ to a good approximation. The key variable in the cross-section is the wave vector

$$
k=q-q^{\prime}
$$

Since the scattering is elastic, $q=q^{\prime}$ and the absolute value of the scattering vector is

$$
k=2 q \sin (\theta / 2)
$$

where $\theta$ is the angle between $q$ and $q^{\prime}$, as shown in Fig (3). A derivation of the following result is outlined later when it is obtained as a particular limit of a much more general expression that incorporates inelastic events and quantum mechanical effects. In this context, we will see that the result given here is, indeed, appropriately labelled as the instantaneous or static value of the cross-section, and the extreme limit 
of the mildly inelastic Compton scattering described in the previous section. With reference to Thomson's formula (1.2), the result is an extension in so far as it applies to an assembly of charges, and allows for a definite polarization of the primary radiation. If $\hat{q}^{\prime}=\left(q^{\prime} / q\right)$ and $\varepsilon$ is the polarization vector of the primary radiation, the effective scattering cross-section for secondary radiation observed in a solid angle $d \Omega$ is,

$$
\frac{\mathrm{d} \sigma}{\mathrm{d} \Omega}=\mathrm{r}_{\mathrm{e}}^{2}<\left|\sum_{\mathrm{j}} \exp \left(\mathrm{ik} \cdot \mathbf{R}_{\mathrm{j}}\right)\right|^{2}>\left\{1-\left(\boldsymbol{\varepsilon} \cdot \hat{\mathbf{q}}^{\prime}\right)^{2}\right\} .
$$

Here, $\boldsymbol{R}_{j}$ is the position of the charge labelled by the index $\mathrm{j}$, and the sum is over all charges.

The angular brackets indicate a time average of the enclosed quantity. However, a principle of statistical physics is that time averaging and thermal averaging are completely equivalent. The averaging appears in $(2,4)$ because the scattering is observed over a time interval large compared with the periods of motion $\left(\sim 1 / \omega_{\mathrm{o}}\right)$ of the charges of the system.

For the wavelength of the primary radiation, there follows from the condition (2.1) the inequality $\lambda \ll(a c / v)$. As for the relative values of $\lambda$ and $a$, both the limiting cases $\lambda>>a$ and $\lambda<<a$ are possible. In both these cases the formula (2.4) simplifies considerably.

In the case $\lambda>>a$, it is permissible in (2.4) to use $\left(k \cdot R_{j}\right) \ll<$, since from (2.3) $k \sim 1 / \lambda$ and $R \sim a$. Replacing the exponential in (2.4) by unity,

$$
\frac{\mathrm{d} \sigma}{\mathrm{d} \Omega}=\mathrm{r}_{\mathrm{e}}^{2} \mathrm{~N}^{2}\left\{1-\left(\varepsilon, \hat{\mathrm{q}}^{\prime}\right)^{2}\right\} .
$$

The scattering cross-section is proportional to the square of the number $N$ of the charges. This finding is in accord with the conclusion drawn in the discussion at the end of the previous sub-section.

The considerations involved in implementing the second limiting case, $\lambda<<a$, are slightly more subtle. In the square of the sum which appears in (2.4), in addition to the square modulus of each term, there appear products of the form $e^{i k .}\left(\boldsymbol{R}_{1}-\boldsymbol{R}_{2}\right)$. In averaging over the motion of the charges, i.e. over their mutual separations, $\boldsymbol{R}_{1}$ $\boldsymbol{R}_{2}$ takes on values in an interval of order a. Since $k \sim 1 / \lambda, \lambda \ll a$, the exponential factors rapidly oscillate in this interval, and the average values vanish. Thus for $\lambda \ll<$ $a$, the effective scattering cross-section is, 


$$
\frac{d \sigma}{d \Omega}=r_{e}^{2} N\left\{1-\left(\varepsilon \cdot \hat{q}^{\prime}\right)^{2}\right\}
$$

The conclusion is that the scattering is proportional to the first power of the number of charges. Note that (2.6) is not applicable for small angles of scattering, $\theta \sim \mathrm{Na}$, since in this case $k \sim \theta / \lambda \sim 1 / a$, and the exponent $k \cdot R$ is not large compared to unity (the scattering is proportional to $N^{2}$ in the limit $\theta \rightarrow 0$ ).

The step leading to (2.6) is an example of the incoherent limit, or incoherent approximation, to the cross-section for scattering from an assembly of particles. There is no surprise in the form of the result since physical intuition is that for sufficiently short wavelengths $(\lambda \ll<$ mean separation of scattering particles) the information obtainable relates to separate particles. For information on particle correlations $\lambda$ should match the relevant length scale, e.g. Bragg scattering.

Perhaps it would be useful to examine a different approach to the incoherent approximation which considers the spread in values of $k$ that occur in a scattering experiment. Let $k_{o}$ and $\Delta k$ denote the mean value and spread in the measured value of the scattering wave vector. On using a guassian function to describe the wave vector resolution, products of the form $e^{i k .}\left(\boldsymbol{R}_{1}-\boldsymbol{R}_{2}\right)$ in the cross-section (2.4) become

$$
\exp \left\{i \mathbf{k}_{\mathrm{o}} \cdot\left(\mathbf{R}_{1}-\mathbf{R}_{2}\right)-\frac{1}{2}(\Delta k)^{2}\left|\mathbf{R}_{1}-\mathbf{R}_{2}\right|^{2}\right\}
$$

In practice, $\Delta k$ is related to $k_{o}$, perhaps a fixed fraction to a good approximation. Hence, the limit $\left(a k_{o}\right) \gg 1$ means a large value for $\Delta k$, in which case the product is negligible unless $\boldsymbol{R}_{1}=\boldsymbol{R}_{2}$, i.e. the main contribution to the cross-section is given by the self-terms. Of course, a large value for $\Delta \mathrm{k}$ means that a large range of wave vectors is sampled, effecting an average of a product term to give,

$$
\int \mathrm{d} \mathbf{k} \mathrm{e}^{\mathrm{ik} \cdot\left(\mathbf{R}_{1}-\mathbf{R}_{2}\right)},
$$

which is vanishingly small unless $\boldsymbol{R}_{1}=\boldsymbol{R}_{2}$ (if the integral is over an infinite range of $\boldsymbol{k}$ the result is a delta-function with an argument $\boldsymbol{R}_{1}-\boldsymbol{R}_{2}$ ).

Let us write (2.4) in a more instructive form. The distribution of the charges in an assembly is partially described by the single particle density function.

$$
\rho_{\mathrm{o}}(\mathbf{r})=\sum_{\mathrm{j}} \delta\left(\mathbf{r}-\mathbf{R}_{\mathrm{j}}\right)
$$


in which $\delta(\boldsymbol{r})$ is a delta-function. From the fundamental property,

$$
\int \mathrm{d} \mathbf{r} \delta(\mathbf{r})=1
$$

it follows that,

$$
\int \mathrm{d} \mathbf{r} \rho_{\mathrm{o}}(\mathbf{r})=\mathrm{N}
$$

Also, if we integrate $\rho_{o}(\boldsymbol{r})$ over a finite volume of space, each $\delta\left(\boldsymbol{r}-\boldsymbol{R}_{j}\right)$ will give either one or zero depending on whether the jth particle is in the volume or not. For the moment we draw attention to the lact that the spatial Fourier transform of the particle density occurs in the cross-section, since

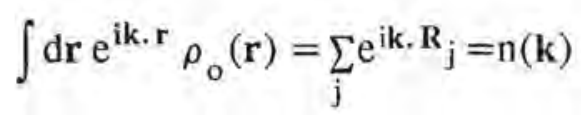

where the final equality defines $n(k)$.

The quantity $\langle n(k)>$ is the thermal average of the Fourier transform of the particle density. It is a function of the parameters which define the state of the target assembly not averaged over in performing the thermal (time) average. Let $\overline{\langle\mathbf{n}(\mathbf{k})\rangle}$ denote the quantity averaged over all parameters. The coherent cross-section is then,

$$
\left(\frac{\mathrm{d} \sigma}{\mathrm{d} \omega}\right)_{\mathrm{coh}}=\mathrm{r}_{\mathrm{e}}^{2}|\overline{\langle\mathrm{n}(\mathbf{k})\rangle}|^{2}\left\{1-\left(\boldsymbol{\varepsilon} \cdot \hat{\mathbf{q}}^{\prime}\right)^{2}\right\}
$$

The difference between the full cross-section (2.4) and the coherent cross-section (2.11) is proportional to the mean-square fluctuation in $n(k)$. This difference is not small for a liquid in the vicinity of the liquid-gas phase transition where fluctuations in the particle density approach macroscopic values, and light scattering displays critical opalescence. For a crystal, $\overline{\left\langle\rho_{0}(\mathbf{r})\right\rangle}$ possesses the full translational symmetry of the crystal structure, and hence $\overline{\langle\mathbf{n}(\mathbf{k})\rangle}$ vanishes unless $\boldsymbol{k}$ coincides with a reciprocal lattice vector. If $\lambda \ll a$, then when we average in (2.10) all terms of the sum vanish, so that the coherent cross-section $=0$. Thus, in this case, scattering is completely incoherent. 


\section{Polarization Effects and Magnetic Scattering Amplitude}

\subsection{Polarization States}

The cross-section (2.4) depends on the polarization of the primary radiation, described by a real unit vector $\varepsilon$. In this section we explore some of the consequences of this dependence, and a more general example in which the beam of secondary radiation has also a definite polarization.

Let us begin by recapping the properties of a classical light wave propagating in the $z$-direction, say. The electric $(\boldsymbol{E})$ and magnetic $(\boldsymbol{H})$ field vectors are perpendicular to the direction of propagation of the light, whence $\boldsymbol{E}=\left(E_{x}, E_{y}, 0\right)$ and for a plane-wave $\boldsymbol{H}=\left(-E_{y}, E_{x}, 0\right)$ or $\mathbf{H}=(\hat{z} \times \mathbf{E})$ where $\hat{z}$ is a unit vector along the $z$-direction. It is often convenient to use a complex notation for the electric field, remembering that in the end the real components lead to observable quantities.

The polarization state of the light wave is directly related to the $\boldsymbol{E}$ vector. Examples of particular interest include:

(a) $E_{y}=0$, plane polarized in the x-direction,

(b) $E_{x}=0$, plane polarized in the y-direction,

(c) $E_{x}=E_{y}$, polarized at $\pi / 4$,

(d) $E_{y}=e^{i \pi / 2} E_{x}$, the $y$-component lags the $x$-component by $90^{\circ}$, and the wave is right circularly polarized,

and

(e) $E_{y}=-i E_{x}$, the wave is left circularly polarized.

Let us choose to employ real polarization vectors; $\varepsilon$ for the primary radiation as in (2.4), and $\varepsilon^{\prime}$ for the secondary radiation. By definition, $\varepsilon \cdot \boldsymbol{q}=\varepsilon^{\prime} \cdot \boldsymbol{q}^{\prime}=0$. The properties of partially polarized radiation are best described in terms of a density matrix. We will not need this formalism for the moment because initially only a few specific features of the Thomson formula will be examined.

Turning to eqn (2.5) for the cross-section of high frequency radiation scattered coherently from an assembly of $N$ charges, let the primary and secondary radiations be directed along $(0,0,1)$ and $(\sin \theta \cos \phi, \sin \theta \sin \phi, \cos \theta)$ as depicted in Fig (3) i.e. $\theta$ and $\phi$ are polar angles for the direction of the secondary radiation, and $\hat{q} \cdot \hat{q}^{\prime}=\cos \theta$. For the definite state of the primary polarization let us choose $\varepsilon=(1,0,0)$. Hence, $\left(\varepsilon . \hat{q}^{\prime}\right)=\sin \theta \cos \phi$ and $(2.5)$ takes the form, 


$$
\frac{\mathrm{d} \sigma}{\mathrm{d} \Omega}=\left(\mathrm{Nr}_{\mathrm{e}}\right)^{2}\left\{1-\sin ^{2} \theta \cos ^{2} \phi\right\} \text {. }
$$

If the primary radiation is unpolarized $\phi$ assumes all possible values since $\boldsymbol{\varepsilon}$ can point in any direction in the $x, y$-plane. The appropriate cross-section is obtained from (3.1) by replacing $\cos ^{2} \phi$ by its average value $(1 / 2)$, in which case,

$$
\frac{\mathrm{d} \sigma}{\mathrm{d} \Omega}=\frac{1}{2}\left(\mathrm{Nr}_{\mathrm{e}}\right)^{2}\left\{1+\cos ^{2} \theta\right\} .
$$

To obtain the total cross-section from (3.2) it is necessary to integrate over the directions of $\hat{q}^{\prime}$, with $d \Omega=\sin \theta d \theta d \phi$, which amounts to replacing $\cos ^{2} \theta$ by $(1 / 3)$ and multiplying by $4 \pi$; the final result is $(1,2)$ applied to $N$ charges.

In the case when the secondary radiation has a definite polarization, described by $\boldsymbol{\varepsilon}^{\prime}$, the cross-section (2.4) takes the value,

$$
\frac{\mathrm{d} \sigma}{\mathrm{d} \Omega}=\mathrm{r}_{\mathrm{e}}^{2}\left\{\boldsymbol{\varepsilon}^{\prime} \cdot \boldsymbol{\varepsilon}\right\}^{2}\left\langle|\mathrm{n}(\mathbf{k})|^{2}\right\rangle .
$$

and a derivation is outlined in $\$ 9$. Here the notation of eqn $(2.10)$ is used. Let the two, mutually orthogonal, states of $\varepsilon^{\prime}$ be chosen as depicted in Fig. (3), for which,

(i) $\varepsilon^{\prime}=(\sin \phi,-\cos \phi, 0)$,

and (ii) $\varepsilon^{\prime}=(\cos \theta \cos \phi, \cos \theta \sin \phi,-\sin \theta)$,

and for the primary radiation $\varepsilon=(1,0,0)$. The state (i) is normal to the plane of scattering, defined by $\boldsymbol{q}$ and $\boldsymbol{q}^{\prime}$, and state (ii) lies in this plane. The corresponding values of the cross-section (3.3) are,

$$
\frac{\mathrm{d} \sigma}{\mathrm{d} \Omega}=\mathrm{r}_{\mathrm{e}}^{2}\left\langle|\mathrm{n}(\mathbf{k})|^{2}\right\rangle\left\{\begin{array}{l}
\sin ^{2} \phi,(\mathrm{i}) \\
\cos ^{2} \theta, \cos ^{2} \phi,(\mathrm{ii})
\end{array}\right.
$$

On summing over the secondary photon polarization we recover the analogue of (3.1).

Observe that when the radiation is scattered through an angle $\theta=90^{\circ}$ the cross-section vanishes for state (ii). Hence, with this geometry, the secondary radiation is polarized in a direction normal to the plane of scattering. The same situation prevails for unpolarized primary radiation, since in this instance averaging 
over $\phi$ in (3.5) merely replaces $\sin ^{2} \phi=\cos ^{2} \phi=(1 / 2)$, and with $\hat{q} \cdot \hat{q}^{\prime}=0$ the secondary radiation is $100 \%$ linearly polarized normal to the plane defined by $q$ and $q^{\prime}$.

\subsection{Density Matrix}

Although several polarization effects are adequately treated by elementary methods, slightly more formalism is required to provide a complete description of polarization contributions to the cross-section and secondary beam. Fortunately, it is possible to derive master formulae for the cross-section and polarization of the secondary beam from which special cases are readily obtained. Hence, at the end of the day there is no need to grapple with the formalism. The master fomulae to which we refer are (3.16) and (3.17). First some material to define concepts and notation, beginning with results that provide a physical interpretation of the density matrix.

Any polarization $\varepsilon$ can be represented as a superposition of two mutually orthogonal polarizations, chosen in some specified manner. These polarizations can be taken to be two mutually perpendicular linear polarizations, or two circular polarizations having opposite directions of rotation. We choose to denote complex vectors of right-hand and left-hand circular polarization by $\varepsilon^{(+)}$and $\varepsilon^{(-)}$, respectively. Seen head-on right-hand circular polarization is counter-clockwise.

In coordinates $\xi, \eta, \zeta$, shown in Fig. (4), with the $\zeta$-axis in the direction of the photon $q$, we take

$$
\varepsilon^{(+)}=-\frac{1}{\sqrt{2}}\left(\varepsilon_{\xi}+i \varepsilon_{\eta}\right)=-\frac{i}{\sqrt{2}}\left(\begin{array}{l}
1 \\
i
\end{array}\right)
$$

and $\boldsymbol{e}^{(-)}=\left(\varepsilon^{(+)}\right)^{*}$. The spin operator for the photon is usually taken to be

$$
\mathbf{S}=\left(\begin{array}{cc}
0 & -\mathbf{i} \\
\mathbf{i} & 0
\end{array}\right)
$$

and

$$
\begin{aligned}
& \mathbf{S} \boldsymbol{\varepsilon}^{(+)}=\boldsymbol{\varepsilon}^{(+)}, \quad \text {;ight }- \text { hand } \\
& \mathbf{S} \boldsymbol{\varepsilon}^{(-)}=-\boldsymbol{\varepsilon}^{(-)} . \quad \text { : left }- \text { hand }
\end{aligned}
$$

The component of angular momentum along the direetion of the momentum vector $q$ (the $\zeta$-axis), called the helicily of the photon, is a conserved quantity. The vectors $\varepsilon^{(t)}$ and $\varepsilon^{(-)}$correspond to the helicity values +1 and -1 , respectively. Thus, the component of the photon angular momentum along the direction of its motion can have only the two values \pm 1 ; the value zero is not possible. 
We employ a density matrix to describe states of partial polarization; applications of this method to photon scattering are reviewed by Tolhoek (1956), McMaster (1961), Newton (1982) and Berestetskii et al. (1982). The polarization density matrix is a tensor $\mu_{\alpha \beta}$ of rank two, in a plane perpendicular to the wave vector $\boldsymbol{q}$ which is the $\xi \eta$-plane with our notation and conventions. The tensor is required to be Hermitian $\mu_{\alpha \beta}=\left(\mu_{\beta} \alpha\right)^{*}$ and normalized such that the trace is unity.

The probability that the photon has any given polarization $\varepsilon$ is determined by the quantity

$$
\varepsilon * \mu \varepsilon
$$

Writing the density matrix in the form

$$
\mu=\left(\begin{array}{ll}
\mu_{11} & \mu_{12} \\
\mu_{21} & \mu_{22}
\end{array}\right),
$$

with $\mu_{21}=\left(\mu_{12}\right) *$, calculation of the quantity (3.8) shows that the components $\mu_{11}$ and $\mu_{22}$ are the probabilities of linear polarizations along the $\xi$ and $\eta$ - axes. Choosing $\varepsilon$ to be the vector $\varepsilon^{(+)}$in (3.6) we find that the probability for right-hand circular polarization

$$
\left(\varepsilon^{(+)}\right) * \mu \quad \varepsilon^{(+)}=\frac{1}{2}\left\{1+\mathrm{i}\left(\mu_{12}-\mu_{21}\right)\right\} .
$$

Next let us consider the two extreme cases of unpolarized and completely polarized photons. For unpolarized photons all directions of polarization are equally probable, and

$$
\mu_{\alpha \beta}=\delta_{\alpha \beta} / 2 .
$$

A photon with a definite polarization $\varepsilon$ is described by the tensor

$$
\mu=\varepsilon \varepsilon^{*},
$$

for then the quantity in (3.8) is unity, as required.

In general, it is convenient to describe partial polarization by means of three real Stokes parameters $P_{1}, P_{2}$, and $P_{3}$, which form a vector $P$. The latter is a purely 
formal step and is done only for the sake of notational convenience. The density matrix $\mu$ can be written,

$$
\mu=\frac{1}{2}\left(\begin{array}{lc}
1+\mathrm{P}_{3} & \mathrm{P}_{1}-\mathrm{iP}_{2} \\
\mathrm{P}_{1}+\mathrm{PP}_{2} & 1-\mathrm{P}_{3}
\end{array}\right)=\frac{1}{2}\{l+\mathrm{P} \cdot \sigma\} .
$$

In the second equality $g$ is the unit matrix, and $\sigma$ are Pauli matrices.

Referring back to our comments on the physical significance of the elements of $\mu$ we see immediately that the parameter $P_{3}$ defines the linear polarization along the $\xi$ - and $\eta$-axis. For the scattering geometry depicted in Fig. $4, P_{3}=1$ corresponds to complete polarization perpendicular to the scattering plane and $P_{3}=-1$ is complete polarization in the scattering plane. Very often, $P_{3}=1$ and $P_{3}=-1$ are referred to as states of $\sigma$ - and $\pi$-polarization, respectively.

The parameter $P_{2}$ represents the degree of circular polarization. From (3.10) the probability of right-hand polarization is $\left(1+P_{2}\right) / 2$, and it is readily shown that the probability for left-hand polarization is $\left(1-P_{2}\right) / 2$. Since these two polarizations correspond to helcities \pm 1 , it is clear that $P_{2}$ is the mean value of the helicity of the photon.

Choosing $\varepsilon=(1, \pm 1) / \sqrt{ } 2$ in the quantity $(3.8)$ reveals that $P_{1}$ describes the linear polarization along directions at angles $\pm \pi / 4$ to the $\xi$-axis. Complete linear polarization of the photon is described by $P_{2}=0, P_{1}^{2}+P_{3}^{2}=1$. In the unpolarized state $P_{1}=P_{2}=P_{3}=0$, while for a completely polarized photon $P_{1}^{2}+P_{2}^{2}+P_{3}^{2}=1$.

\subsection{Scattering Amplitude}

The scattering amplitude operator $G$ is written as a $2 \times 2$ matrix by constructing four elements for the two values of $\varepsilon$ and $\varepsilon^{\prime}$. Our scheme for this is defined in Fig. 5, in which the diagonal elements $G_{11}$ and $G_{22}$ are amplitudes for both $\varepsilon$ and $\varepsilon^{\prime}$ perpendicular $(\sigma-\sigma)$ and parallel $(\pi-\pi)$ to the scattering plane, respectively. The element $G_{21}$ is the amplitude for the event in which the initial polarization $\varepsilon$ is perpendicular to the scattering plane and the final polarization $\varepsilon^{\prime}$ is parallel to the plane $(\sigma-\pi)$, while $G_{12}$ describes the event in which the polarizations are in the reverse order $(\pi-\sigma)$.

It is convenient to write the matrix $G$ in the form

$$
\mathbf{G}=\beta \vartheta+\alpha \cdot \sigma=\left(\begin{array}{cc}
\beta+\alpha_{3} & \alpha_{1}-i \alpha_{2} \\
\alpha_{1}+j \alpha_{2} & \beta-\alpha_{3}
\end{array}\right)
$$


where $\beta$ is a scalar quantity, $\alpha=\left(\alpha_{1}, \alpha_{2}, \alpha_{3}\right)$, and $g$ is the unit matrix. The convenience arises when we calculate the cross-section

$$
\frac{\mathrm{d} \sigma}{\mathrm{d} \Omega}=\mathrm{re}_{\mathrm{e}}^{2} \mathrm{Tr} \cdot\left\{\mu \mathbf{G}^{+} \mathbf{G}\right\},
$$

where the trace operation is taken with respect to the photon polarization states. A similar situation arises in the calculation of Stokes parameters for the secondary photon beam $P^{\prime}$ obtained from the relation

$$
\left(\frac{d \sigma}{d \Omega}\right) \mathbf{P}^{\prime}=r_{e}^{2} \operatorname{Tr} \cdot\left\{\mu \mathbf{G}^{+} \sigma \mathbf{G}\right\} .
$$

The trace operations in (3.14) and (3.15) entail quite a bit of algebra and so it is not given here; simpler examples of what is involved are outlined in $\$ \S 14,15$. Inserting the expressions (3.12) and (3.13) in (3.14) and performing the trace operation yields

$$
\frac{\mathrm{d} \sigma}{\mathrm{d} \Omega}=\mathrm{re}_{\mathrm{e}}^{2}\left[\alpha^{+} \cdot \alpha+\beta^{+} \beta+\beta^{+}(\alpha \cdot \mathbf{P})+\left(\alpha^{+} \cdot \mathbf{P}\right) \beta+i \mathbf{P} \cdot\left(\alpha^{+} \times \alpha\right)\right] .
$$

while for $P^{\prime}$ we obtain,

$$
\begin{gathered}
\left(\frac{\mathrm{d} \sigma}{\mathrm{d} \Omega}\right) \mathbf{P}^{\prime}=\mathrm{re}_{\mathrm{e}}^{2}\left[\beta^{+} \alpha+\alpha^{+} \beta-\mathrm{i}\left(\alpha^{+} \times \alpha\right)+\beta^{+} \beta \mathbf{P}+\alpha^{+}(\alpha \cdot \mathbf{P})+\left(\alpha^{+}, \mathbf{P}\right) \alpha-\mathbf{P}\left(\alpha^{+} \cdot \alpha\right)\right. \\
\left.+i \beta^{+}(\alpha \times \mathbf{P})+i\left(\mathbf{P} \times \alpha^{+}\right) \beta\right] .
\end{gathered}
$$

In deriving (3.16) and (3.17) we have assumed that $\beta$ and $\alpha$ are quantum-mechanical operators that do not commute. The expressions for $(\mathrm{d} \sigma / \mathrm{d} \Omega)$ and $\mathbf{P}^{\prime}$ are in the form of products of operators, and to obtain results for the interpretation of experiments (Bragg diffraction, Compton scattering, etc) appropriate matrix elements have to be formed, which are topics taken up in later seclions. For the moment, we emphasize that (3.16) and (3.17) provide general statements with regard to the polarization dependence of the cross-section, and the polarization state of the secondary beam, and apply to resonant and nonresonant events.

The expressions for $\alpha_{1}, \alpha_{2}, \alpha_{3}$ and $\beta$ appropriate for diffraction and spectroscopy can be expressed in terms of three atomic quantities that have immediate physical interpretations. One quantity is the spatial Fourier transform of the particle density, $n(k)$, defined in (2.10). The remaining two quantities relate to magnetic properties, and they are the spin density 


$$
\mathbf{S}(\mathbf{k})=\sum_{\mathrm{j}} \mathbf{s}_{\mathrm{j}} \exp \left(\text { ik. } \mathbf{R}_{\mathrm{j}}\right)
$$

where $s_{j}$ is the spin operator for the $j^{\prime} t h$ electron, and

$$
\mathbf{Z}(\mathbf{k})=(\mathbf{i} / \hbar \mathbf{q}) \sum_{j} \exp \left(\mathbf{i k} \cdot \mathbf{R}_{j}\right)\left\{\left(\hat{\mathbf{q}}-\hat{\mathbf{q}}^{t}\right) \mathbf{x p}_{\mathbf{j}}\right\}
$$

where $\boldsymbol{p}$ is the momentum operator conjugate to $\boldsymbol{R}$, and $\hat{\boldsymbol{q}}$ and $\hat{\boldsymbol{q}}^{\prime}$ are unit vectors. Note that $S(k)$ and $Z(k)$ are dimensionless. The physical significance of $Z(k)$ is revealed through an identity, required in the evaluation of the elastic cross-section, that relates $Z(k)$ to an orbital angular momentum operator

$$
\begin{aligned}
& \mathbf{T}(\mathbf{k})=\left(1 / \mathrm{i} \hbar \mathbf{k}^{2}\right) \sum_{\mathrm{j}} \exp \left(\mathrm{ik} \cdot \mathbf{R}_{\mathrm{j}}\right)\left(\mathbf{k} \times \mathbf{p}_{\mathbf{j}}\right) \\
& =-\left(1 / 2 \mu_{\mathrm{B}}\right) \int \mathrm{d} \mathbf{r} \exp (\mathrm{i} \cdot \mathbf{k} \cdot \mathbf{r})\left\{\hat{\mathbf{k}} \times\left(\mathbf{M}_{\ell}(\mathbf{r}) \times \hat{\mathbf{k}}\right)\right\}
\end{aligned}
$$

in which $\boldsymbol{M}_{I}(\boldsymbol{r})$ is the orbital magnetization density; a proof of the second equality is given by Balcar and Lovesey (1989). The identity in question is $\left(q=q^{\prime}\right)$,

$$
2\left(\hat{\mathbf{q}}+\cos \theta \hat{\mathbf{q}}^{\prime}\right) \cdot \mathbf{Z}(\mathbf{k})=-\frac{\mathbf{k}^{2}(1+\cos \theta)}{q^{2}}\left(\hat{\mathbf{q}}+\hat{\mathbf{q}}^{\prime}\right) \cdot \mathbf{T}(\mathbf{k}) .
$$

Here, $\theta$ is the angle between the primary and secondary photon beams, i.e. $\hat{\boldsymbol{q}} \cdot \hat{\boldsymbol{q}}^{\prime}=\cos \theta$. Note that the sums in (3.18) and (3.19) are over all electrons in the target assembly. Matrix elements of $S(k)$ and $Z(k)$, or $M_{l}$ needed in the cross-section for a magnetic material will, however, involve only the fraction of electrons that are unpaired, e.g. for the localized atomic ion model for a rare earth magnet, core electrons will not contribute to the matrix elements in the first approximation. Core electrons are manifest in various resonant spectroscopies, and several examples are examined in $\$ \S 14,15$.

The expressions for $\alpha$ and $\beta$ are found from the results (11.5) and (11.6) $\left(\tau=\mathrm{E} / m c^{2}\right)$

$$
\begin{aligned}
& \alpha_{1}=-\frac{i}{2} \tau \mathbf{S}(\mathbf{k}) \cdot\left\{(2 \mathrm{a}-\mathrm{b}-\cos \theta) \hat{\mathbf{q}}+(1+\mathrm{b} \cos \theta-2 \mathrm{a}) \hat{\mathbf{q}}^{\prime}\right\}-\frac{1}{2} \tau\left(\hat{\mathbf{q}}-\hat{\mathbf{q}}^{\prime}\right) \cdot \mathbf{Z}(\mathbf{k}) \\
& \alpha_{2}=\frac{1}{2} \tau \mathbf{S}(\mathbf{k}) \cdot\left\{(\cos \theta-b) \hat{\mathbf{q}}+(b \cos \theta-1) \hat{\mathbf{q}}^{\prime}\right\}+\frac{1}{2} \tau\left(\hat{\mathbf{q}}+\hat{\mathbf{q}}^{\prime}\right) \cdot \mathbf{Z}(\mathbf{k})
\end{aligned}
$$




$$
\begin{aligned}
& \alpha_{3}=\frac{1}{2}(1-\cos \theta) n(\mathbf{k})+\frac{i}{2} \tau\left(\hat{\mathbf{q}} \times \hat{\mathbf{q}}^{\prime}\right) \cdot\{(\mathrm{b}+1-2 \mathrm{a}) \mathbf{S}(\mathbf{k})-\mathbf{Z}(\mathbf{k})\} \\
& \boldsymbol{\beta}=\frac{1}{2}(1+\cos \theta) n(\mathbf{k})+\frac{i}{2} \tau\left(\hat{\mathbf{q}} \times \hat{\mathbf{q}}^{\prime}\right) \cdot\{\mathbf{Z}(\mathbf{k})-(b+1) \mathbf{S}(\mathbf{k})\},
\end{aligned}
$$

where $b=\left(q^{\prime} / q\right)$ and $a=(1+b) / 2$, and $a=b=1$ for elastic events. Notice that $\alpha_{1}$ and $\alpha_{2}$ are purely magnetic in character $\left(\alpha_{1}=\alpha_{2}=0\right.$ for $\left.\tau=0\right)$, and the expressions for $\alpha$ and $\beta$ are correct to first-order in $\tau$.

The foregoing expressions for $\alpha$ and $\beta$ taken together with (3.16) and (3.17) enable us to calculate the cross-section to order $\tau$. In the event that terms of order $\tau^{2}$ are needed then the amplitude $G$ must be calculated to the same order. One route by which this can be achieved is to use expressions derived by Grotch et al. (1983). The same remarks apply to the calculation of the secondary polarization from (3.17). However, the term of order $\tau^{2}$ in $\mathbf{G}$ will contribute to the cross-section at order $\tau^{2}$ a term linear in the charge and quadratic in spin and linear momentum. The product of matrix elements of this operators will usually be very small compared to the matrix elements that multiply $\tau^{2}$ in the cross-section derived from (3.22)

We have remarked that for elastic scattering $\mathbf{Z}(\mathbf{k})$ is proportional to the orbital magnetization in the sample. Looking at $\alpha$ and $\beta$ one is struck by the lack of any symmetry in the spin and orbital contributions. The origin of this state of affairs can be traced back to the perturbative calculation of $\mathbf{G}$, about which we have more to say in $\S 9$. It is found that the orbital contribution arises from second-order processes, the same ones that generate Raman scattering. On the other hand, the spin contribution is the sum of terms that arise in first-and second-order processes. More on this topic is found in the review articles by de Bergevin and Brunel (1986) and Lovesey (1987a). A consequence of the lack of symmetry in the spin and orbital contributions is that scattering geometries exist were one or the other contribution is absent, a feat not possible in neutron scattering (Balcar and Lovesey 1989, Lander and Stirling 1992). 


\section{Partial Differential Cross-Section}

The master expression for the interpretation of non-resonant scattering experiments is a partial differential cross-section, which gives the fraction of particles (photons) of incident energy $\mathrm{E}$ scattered into an element of solid angle $d \Omega$ with an energy between $E^{\prime}$ and $E^{\prime \prime}+d E^{\prime}$. The cross-section is denoted by

\section{$d^{2} \sigma / d \Omega d E^{\prime}$}

and has the dimension of (area/energy). In a more general language, the cross-section is a response function; it measures the response of the target assembly to a probe characterized by a wave vector $k=q-q^{\prime}\left(E=\hbar c q, E^{\prime}=\hbar c q^{\prime}\right)$ and frequency $\omega=(E$ $\left.E^{\prime}\right) / \hbar$. The probe can couple to the particle density, as illustrated by the examples of Thomson, Rayleigh and Compton processes introduced in previous sections, or, as will be demonstrated, to the magnetization density.

The fundamental physical properties of a response function are that it is purely real, and positive or possibly zero; an imaginary or negative response function is a signature of an assembly unstable with respect to perturbations (fluctuations) induced by the probe.

The specific heat of an assembly can be viewed as a bulk response function. The specific heat at constant pressure, for example, is proportional to the mean-square fluctuation in the entropy, while the static magnetic susceptibility is proportional to the mean-square fluctuation in the total magnetization (both quantities diverge at the temperature for a continuous phase transition). In this sense, cross-sections, for neutron or photon scattering, are differential response functions which displayed as a function of $k$ and $\omega$ contain a wealth of information, and generally more than a bulk response.

Let us begin a closer inspection of the cross-section and the information it contains by using the Thomson formula in the form (3.3). Here, information on the target assembly is contained in $\left\langle|n(k)|^{2}\right\rangle$, while the other factors in the formula relate to the specific nature of the scattering process, or in other words the nature of the coupling of the proble to the target. Recall that angular brackets denote a time average of $|n(k)|^{2}$. This average is completely equivalent to a thermal average expressed in terms of diagonal matrix elements of $|n(\mathbf{k})|^{2}$ weighted by thermal (Boltzmann) factors. If the target assembly is described by a Hamiltonian $\mathcal{H}$, whose eigenstates are labelled by quantum numbers $\{\mu\}$, with

$$
\nVdash\left|\mu>=E_{\mu}\right| \mu>,
$$


then

$$
\langle\ldots\rangle=(1 / z) \sum_{\mu} \exp \left(-E_{\mu} / k_{B} T\right)\langle\mu|\ldots| \mu\rangle
$$

where the partition function,

$$
\mathrm{Z}=\sum_{\mu} \exp \left(-\mathrm{E}_{\mu} / \mathrm{k}_{\mathrm{B}} \mathrm{T}\right)
$$

Very often it is useful to express the sum of diagonal matrix elements in (4.2) as a trace of the product of the operator to be averaged and $\exp \left(-\Re_{1} / k_{B} T\right)$. Note that the trace is invariant to a cyclic permutation of operators, i.e. $\operatorname{Tr} .(A B)=\operatorname{Tr}$. $(B A)$. The sums in (4.2) and (4.3) are over all states of $\mathcal{H}$, both in the discrete and continuous parts of the spectrum. For states in the continuum a sum is replaced by an appropriate integral.

The partial differential cross-section for the Thomson process is,

$$
\frac{d^{2} \sigma}{d \Omega d E^{\prime}}=r^{2}\left(\frac{E^{\prime}}{E}\right)\left\{\varepsilon \cdot \varepsilon^{\prime}\right\}^{2}\left\langle|n(k)|^{2}\right\rangle \delta(\hbar \omega)
$$

Here, the delta-function expresses conservation of energy. In the present case, the scattering is elastic $\omega=\left(E-E^{\prime}\right) / \hbar=c\left(q-q^{\prime}\right)=0$, and coherent since the formula is based on classical mechanics. Because $E=E^{\prime}$ the kinematic factor $\left(E^{\prime} / E\right)=1$, and hence it is redundant in the present case. This factor arises in the general formulation from the density of final photon states divided by the primary photon flux.

If inelastic events contribute to photon scattering from the charge density, and they will in general, the appropriate result for the cross-section is,

$$
\frac{\mathrm{d}^{2} \sigma}{\mathrm{d} \Omega \mathrm{dE} \mathrm{E}^{\prime}}=\mathrm{r}_{\mathrm{e}}^{2}\left(\mathrm{q}^{\prime} / \mathrm{q}\right)\left\{\boldsymbol{\varepsilon}, \boldsymbol{\varepsilon}^{\prime}\right\}^{2} \sum_{\mu \mu^{\prime}} \mathrm{p}_{\mu}\left|\left\langle\mu^{\prime}|\mathrm{n}(\mathbf{k})| \mu\right\rangle\right|^{2} \delta\left(\hbar \omega+\mathrm{E}_{\mu}-\mathrm{E}_{\mu^{\prime} \cdot}\right) .
$$

where, for convenience, we have introduced,

$$
\mathrm{p}_{\mu}=\exp \left(-\mathrm{E}_{\mu} / \mathrm{k}_{\mathrm{B}} \mathrm{T}\right) / \mathrm{Z}
$$

Note that the right-hand side is purely real, and positive or possibly zero. 
To recover the Thomson formula (4.4) from (4.5) it is necessary to eliminate in the delta-function the dependence on $E_{\mu}$ and $E_{\mu^{\prime}}$, for when this is possible the closure relation,

$$
\sum_{\mu^{\prime}}\left|\mu^{\prime}\right\rangle\left\langle\mu^{\prime}\right|=1,
$$

can be used to reduce (4.5) and, with the aid of (4.2), obtain (4.4). In (4.7) the sum is over the entire spectrum of states for the target assembly. We can eliminate $E_{\mu}$ and $E_{\mu}$ from the delta-function by choosing a sufficiently large primary photon energy, such that $E+E_{\mu} \sim E$ for the maximum $E_{\mu}$ at which the matrix element is significant. When this is achieved, it follows that $E^{\prime}+E_{\mu^{\prime}} \sim E^{\prime}$, and the scattering process is elastic, to a good approximation. This argument is a rewording of the argument used in $\$ 1.2$ to establish the condition, and its ramifications, under which the Thomson process is observed. Note that Bragg scattering is strictly elastic, whereas the Thomson process emerges with increasing primary photon energy as the dominant process. A subsequent section is devoted to Compton scattering, which is mildly inelastic.

In recognition of the factorization of the cross-section (4.5) into factors that depend on the nature of the probe-target coupling, and factors which express the dependence on properties of the target assembly, it is customary to introduce a response function for $N$ charges,

$$
\mathrm{S}(\mathbf{k}, \omega)=\frac{1}{\mathrm{~N}} \sum_{\mu \mu^{\prime}} \mathrm{p} \mu\left|\left\langle\mu^{\prime}|\mathrm{n}(\mathbf{k})| \mu\right\rangle\right|^{2} \delta\left(\hbar \omega+\mathrm{E}_{\mu}-\mathrm{E}_{\mu^{\prime}}\right),
$$

which has the dimension (1/energy). The non-relativistic partial differential crosssection for charge scattering (4.5) is,

$$
\frac{\mathrm{d}^{2} \sigma}{\mathrm{d} \Omega \mathrm{dE} \mathrm{E}^{\prime}}=\mathrm{Nr}_{\mathrm{e}}{ }^{2}\left(\mathrm{q}^{\prime} / \mathrm{q}\right)\left\{\boldsymbol{\varepsilon}, \boldsymbol{\varepsilon}^{\prime}\right\}^{2} \mathrm{~S}(\mathrm{k}, \omega)
$$

The function $S(k, \omega)$ was introduced by Van Hove in the literature on neutron scattering, and it is also referred to as a dynamic structure factor or scattering law. In the case of photon scattering from the charge distribution in materials, to which (4.8) and (4.9) relate, $S(k, \omega)$ is proportional to the inverse of the dielectric function (Lovesey 1986, Callaway 1991). For magnetic scattering, $S(k, \omega)$ is proportional to the frequency and wave vector dependent susceptibility, which might be guessed 
since the bulk susceptibility is proportional to the mean-square fluctuation in the magnetization and the probe (neutron or photon beam) creates such a fluctuation.

The validity of the formula for the non-relativistic cross-section merits some comment. A simple derivation of (4.5) in the framework of quantum mechanics is based on the use of Fermi's Golden Rule for transition rates, here the transition $|\mu\rangle \rightarrow\left|\mu^{\prime}\right\rangle$ caused by the primary radiation. This is equivalent to use of first-order perturbation theory. Couched in a slightly different, but useful, language it is assumed that the response of the target assembly is proportional to the strength of the probe, i.e. it is a linear response. The same assumption underlies the interpretation of most experiments performed in condensed matter and materials research. In particular, linear response theory for systems close to thermal equilibrium (not systems which display chaotic or turbulent behaviour) is the bed-rock for the interpretation of (non-relativistic) electron, neutron and photon scattering experiments, and relaxation and resonance experiments using implanted positive muons, Mössbauer isotopes, NMR, and related methods (Lovesey 1986). An immediate benefit is a common language for the interpretation of data obtained by a wide range of techniques, and a direct interface between data, results from computer simulations, and theoretical work. 


\section{Correlation Functions}

There is more than mere nicety to be gained from expressing $S(\boldsymbol{k}, \omega)$ in terms of time-dependent correlation functions; they provide a compact form for somewhat unwieldy formulae, such as (4.8), a natural vehicle through which to express physical intuition regarding properties of matter, and an attractive quantity for theoretical treatment. Mindful to use the least cumbersome presentation, the discussion of correlation functions is couched in terms of charge scattering. The results reviewed hold also for the magnetic operators encountered in the previous section. In the case of non-relativistic, non-resonant photon scattering by charges, the relevant correlation function is,

$$
\langle\mathrm{n}+(\mathbf{k}) \mathrm{n}(\mathrm{k}, \mathrm{t})\rangle \text {. }
$$

where $n(\boldsymbol{k})$ is defined in (2.10), and $n(\boldsymbol{k}, t)$ is a Heisenberg operator,

$$
\mathrm{n}(\mathbf{k}, \mathrm{t})=\exp (\mathrm{i} t \mathscr{H} / \hbar) \mathrm{n}(\mathbf{k}) \exp \left(-\mathrm{i} \mathfrak{H} / \hbar_{)}\right)
$$

in which $\mathcal{H}$ is the Hamiltonian for the target assembly of charged particles and their environment, and $t$ has the dimension of time. The operator $\exp ($ it Ylt/ $\hbar)$ is a time-shift operator, and the operation on $n(k)$ defined by the right-hand side of $(5.2)$ amounts to a unitary transformation.

The response function $S(k, \omega)$ delined by $(4.8)$ is the time Fourier transform of (5.1),

$$
S(k, \omega)=\left(\frac{1}{2 \pi h N}\right) \int_{-\infty}^{\infty} d t e^{-i \omega t}\left\langle n^{+}(k) n(k, 1)\right\rangle
$$

To establish (5.3) note that,

$$
\left|\left\langle\mu^{\prime}|\mathbf{n}(\mathbf{k})| \mu\right\rangle\right|^{2}=\left\langle\mu\left|\mathbf{n}^{+}(\mathbf{k})\right| \mu^{\prime}\right\rangle\left\langle\mu^{\prime}|\mathbf{n}(\mathbf{k})| \mu\right\rangle
$$

where $n^{+}(k)$ is the Hermitian conjugate of $n(k)$,

$$
\left\langle\mu^{\prime}|\mathrm{n}(\mathbf{k}, \mathrm{t})| \mu\right\rangle=\left\langle\mu^{\prime}|\mathrm{n}(\mathbf{k})| \mu\right\rangle \exp \left\{\frac{\mathrm{ii}}{l^{\prime}}\left(E_{\mu^{*}}-\mathrm{E}_{\mu}\right)\right\}
$$

and the representation, 


$$
\delta(x)=\frac{1}{2 \pi} \int_{-\infty}^{\infty} d t e^{\mathrm{ixt}}
$$

is used to express the delta-function in (4.8) in a form that enables use of (5.5), and this leads to $S(k, \omega)$ written in terms of a Fourier transform. Since $S(k, \omega)$ is a measured response function it is purely real, yet (5.3) contains manifestly complex quantities. In fact, correlation functions are usually complex quantities, the imaginary part coming from quantum aspects (it vanishes for $\hbar=0$ ). To establish that the righthand side of (5.3) is purely real, use the identity (for a derivation of (5.7) - (5.9) see, for example, Lovesey 1986),

$$
\left\langle n^{+}(\mathbf{k}) n(k, t)\right\rangle^{*}=\left\langle n^{+}(\mathbf{k}, t) n(k)\right\rangle
$$

together with,

$$
\left\langle\mathrm{n}^{+}(\mathbf{k}, \mathrm{t}) \mathrm{n}(\mathbf{k})\right\rangle=\left\langle\mathrm{n}^{+}(\mathbf{k}) \mathrm{n}(\mathbf{k},-\mathrm{t})\right\rangle \text {. }
$$

which expresses the physical requirement that the target assembly is a stationary system, since more generally,

$$
\left\langle n^{+}\left(k, t_{0}\right) n\left(k, t+t_{0}\right)\right\rangle=\left\langle n^{+}(k) n(k, t)\right\rangle
$$

for an arbitrary $t_{O}$, and (5.8) results from the choice $t+t_{O}=0$. A correlation function is not usually invariant with respect to an interchange of the operators. The identity,

$$
\left\langle\mathrm{n}^{+}(\mathbf{k}, \mathrm{t}) \mathrm{n}(\mathbf{k})\right\rangle=\left\langle\mathrm{n}(\mathbf{k}) \mathrm{n}^{+}(\mathbf{k}, \mathrm{t}+i \hbar \beta)\right\rangle
$$

where $\beta=\left(1 / \mathrm{k}_{\mathrm{B}} \mathrm{T}\right)$ demonstrates that this feature is rooted in the quantum properties of the correlation function. From (5.10),

$$
\mathrm{S}(\mathbf{k}, \omega)=\mathrm{e}^{\hbar \omega \beta} \mathrm{S}(-\mathbf{k},-\omega)
$$

which is often referrred to as the condition of detailed balance. For some systems $S(k, \omega)$ depends on $k=|k|$, in which case $(5.11)$ provides the relation between processes in which energy is transferred to and removed from the target assembly; in our notation $\omega>0$ corresponds to a transfer of energy from the primary radiation to the target. 
If the target assembly is spatially homogeneous then $S(k, \omega)$ depends on $k$ and not $k$, i.e. it depends on two and not four variables. The response function for a target not subject to a magnetic field is invariant with respect to the sign of $k$. This property follows from the behaviour under time reversal of an auto-correlation function constructed from a Hermitian operator (Lovesey 1986). Since observables are formed by Hermitian operators, such as the particle density (2.7), this behaviour applies to measured response functions.

The strictly clastic component of the response function is derived from the infinite time limit of the correlation function, namely,

$$
\mathrm{NS}(\mathbf{k}, \omega)=\delta(\hbar \omega)\left\langle\mathrm{n}^{+}(\mathbf{k}) \mathrm{n}(\mathbf{k}, \mathrm{t}=\infty)\right\rangle .
$$

The law of increase in entropy, or loss of information, requires that, for a bulk assembly, there is no correlation between processes that are well separated in time. Consequently,

$$
\begin{aligned}
\left\langle\mathrm{n}^{+}(\mathbf{k}) \mathrm{n}(\mathbf{k}, \infty)\right\rangle & =\left\langle\mathrm{n}^{+}(\mathbf{k})\right\rangle\langle\mathrm{n}(\mathbf{k}, \infty)\rangle \\
& =|\langle\mathrm{n}(\mathbf{k})\rangle|^{2},
\end{aligned}
$$

where the second equality is valid for stationary systems in which $\langle n(k, t)\rangle=\langle n(k, 0)\rangle$ for any value of $t$. Note that simple model systems in which there is no mechanism for damping might not display loss of correlation between events separated by a large time interval. But such behaviour is artificial, all real bulk systems follow the law of increase in entropy, if only because there is a weak coupling to a second body, or reservoir,

The result (5.13) should be compared with (2.11). In the latter case, account is taken of the fact that $\langle n(k)\rangle$ might depend on parameters which describe disorder. The quantity $\overline{\langle\mathrm{n}(\mathrm{k})\rangle}$ is the charge density averaged thermally and with respect to all other parameters required to describe the target assembly. Coherent (elastic) scattering is determined by,

$$
\left.|\overline{\langle n(k)}\rangle\right|^{2}
$$

One example is Bragg scattering from a crystal. The remaining part of (5.13), 


$$
\overline{\left.|\langle\mathbf{n}(\mathbf{k})\rangle-\overline{\langle\mathrm{n}(\mathbf{k})}\rangle\right|^{2}}
$$

is (elastic) diffuse scattering, which arises from defects, compositional effects, etc.

The opposite limit to the time-averaged scattering, described by $(5,12)$, is the static or instantaneous approximation. In this case, the correlation function in $S(k, \omega)$ is replaced by its value of $t=0$, namely $\left\langle|n(k)|^{2}\right\rangle$. Again, the scattering is elastic,

$$
\mathrm{N} \mathrm{S}(\mathbf{k}, \omega)=\delta(\hbar \omega)\left\langle|\mathrm{n}(\mathbf{k})|^{2}\right\rangle
$$

The difference between (5.12) and (5.16) is due to thermal fluctuations in the particle density. The result (5.16) is Thomson scattering, c.f. (4.4).

\section{Spontaneous Emission}

There is, perhaps, something of a need to rationalize the decision to discuss spontaneous emission of photons in an article otherwise devoted to photon scattering, or second-order processes. First of all, it is a relatively easy topic which provides a somewhat gentle introduction to more complex topics in response theory, like the generalized Kramers-Heisenberg formula. Secondly, a discussion of spontaneous emission introduces concepts which are central to the main subject of the text, namely the photon interaction with spin and orbital magnetic moments.

Section 3 explores some features of polarization phenomena in photon scattering, in the course of which there is mention of the electric $(\boldsymbol{E})$ and magnetic $(\boldsymbol{H})$ fields associated with a classical light wave. These can be obtained from a vector potential $\boldsymbol{A}$, e.g. $\boldsymbol{H}=\operatorname{curl} \boldsymbol{A}$, which is the key quantity in describing the interaction of photons with charge carriers. Classically, the Hamiltonian for a particle of charge $\mathrm{e}$ and mass $\mathrm{m}^{*}$ in an electromagnetic field is

$$
\frac{1}{2 m^{*}}\left\{\mathbf{p}-\frac{e}{c} \mathbf{A}(\mathbf{r}, t)\right\}^{2}+e \varphi(\mathbf{r}, t)+V_{o}(\mathbf{r}, t)
$$

where $\varphi(\boldsymbol{r}, t)$ is the scalar potential and $V_{o}(\boldsymbol{r}, t)$ describes all other potentials seen by the particle. The velocity of a charged particle in the presence of an electromagnetic field is seen to be $\left(\mathbf{p}-\frac{\mathrm{e}}{\mathrm{c}} \mathbf{A}\right) / \mathrm{m}^{*}$. It is convenient to work in the transverse gauge in which $\varphi=0$, and $\boldsymbol{A}$ is divergence-free, so $\operatorname{div} \boldsymbol{A}=0$. The interaction between a charge and radiation field is evidently described by , 


$$
V=-\frac{e}{2 m^{*} c}\{\mathbf{p} \cdot \mathbf{A}(\mathbf{r}, t)+\mathbf{A}(\mathbf{r}, t) \cdot \mathbf{p}\}+\frac{\mathrm{e}^{2}}{2 \mathrm{~m}^{*} \mathrm{c}^{2}} \mathrm{~A}^{2}(\mathbf{r}, \mathrm{t})
$$

where for convenience later on the $\boldsymbol{p} . \boldsymbol{A}$ terms have been written in a symmetric form. The next step is to generalize $V$ for a system of many particles, say $N$, interacting with radiation. Let $\mathrm{j}$ index the particles, as in the particle density function defined by eqn (2.7), from which

$$
\sum_{\mathbf{j}} \mathrm{A}^{2}\left(\mathbf{R}_{\mathbf{j}}\right)=\int \mathrm{d} \mathbf{r} \rho_{\mathrm{o}}(\mathbf{r}) \mathrm{A}^{2}(\mathbf{r}) .
$$

To write the $p . A$ terms for many particles in a similar form, define a current density function,

$$
\mathbf{J}(\mathbf{r})=\frac{1}{2 \mathrm{~m}^{*}} \sum_{\mathbf{j}}\left\{\mathbf{p}_{\mathrm{j}} \delta\left(\mathbf{r}-\mathbf{R}_{\mathbf{j}}\right)+\delta\left(\mathbf{r}-\mathbf{R}_{\mathbf{j}}\right) \mathbf{p}_{\mathbf{j}}\right\}
$$

in terms of which the first part of $V$ is,

$$
-\frac{e}{c} \int d \mathbf{r} \mathbf{J}(\mathbf{r}) \cdot \mathbf{A}(\mathbf{r}, t)
$$

It can be shown that, in a quantum mechanical treatment these expression remain valid with $\boldsymbol{A}(\boldsymbol{r}, t)$ expressed as a linear combination of photon creation and annihilation operators. Thus, the $p . A$ terms are linear in photon operators, whereas the $A^{2}$ term is quadratic in these operators.

To complete the quantum mechanical description the interaction of the electron spin with the magnetic field $H=\operatorname{curl} A$ must be added to the interaction operator $V$. The interaction with the spins $\left\{s_{j}\right\}$ is

$$
\mathrm{V}_{\mathrm{S}}=-\mathrm{g}_{\mathrm{o}} \mu_{\mathrm{B}} \sum_{\mathrm{j}} \mathbf{s}_{\mathrm{j}} \cdot \mathbf{H}\left(\mathbf{R}_{\mathrm{i}}\right)
$$

where $g_{O}$ is the gyromagnetic factor $\sim 2.0$, and $\mu_{B}$ the Bohr magneton. Since the spin term is linear in $A$ it can be regarded as producing an additional contribution to the current $J$ in (6.4). In fact, in a consistent theory of electromagnetic fields the interaction naturally appears in the form given in (6.4), with $J$ the sum of the current and spin densilies, c.f. Berestetskii el al. (1982). However, for the present purpose of discussing spontaneous emission nothing but elegance is lost in the foregoing hotchpot treatment. 
To treat absorption or emission, the intial and final photon states differ by one in the number of photons. Since (6.4) and (6.5) are linear in $A$, a linear combination of creation and annihilation operators, there is a finite value of the matrix elements between states which differ by one in the number of photons. The quadratic term $A^{2}$ in (6.2) makes no contribution to absorption and emission processes in lowest order because it changes the number of photons by either 0 or \pm 2 .

The transition rate for spontaneous emission is calculated to lowest order in the fine structure constant $\alpha=\left(e^{2} / \mathrm{hc}\right)$. This is achieved by use of Fermi's Golden Rule for transition rates,

$$
\frac{2 \pi}{h} \delta\left(\mathrm{E}_{i}-\mathrm{E}_{f}\right)|\langle f|\mathrm{~V}| i\rangle|^{2}
$$

in which $|i\rangle,|\mathrm{f}\rangle$ denote the initial and final states with eneregies $E_{i}$ and $E_{f}$, and $V$ is the sum of (6.4) and (6.5). The state $|i\rangle$ is a composite state in which there is no photon and the atom, more generally an assembly of charges, is in its initial state $|\mu\rangle$, with energy $E_{\mu}$, and $|f\rangle$ is a state with one photon and an atomic state $\left|\mu^{\prime}\right\rangle$ with energy $E_{\mu^{\prime}}$. Let the photon have an energy hcq, then in (6.6)

$$
\mathrm{E}_{f}-\mathrm{E}_{i}=\mathrm{E}_{\mu^{\prime}}+\hbar \mathrm{cq}-\mathrm{E}_{\mu}
$$

The matrix element of $A(r, t)$ is denoted by $\langle 1|A| 0\rangle$ where the notation indicates that the initial and final photon states contain, respectively, 0 and 1 photons. With our choice of units,

$$
\langle 1|A(\mathbf{r}, t)| 0\rangle=\varepsilon \exp \{i \omega t-i q \cdot \mathbf{r}\}\left(2 \pi \hbar c^{2} / \Omega \omega\right)^{\frac{1}{2}},
$$

where $\varepsilon$ is the polarization vector associated with the emitted photon that has a wave vector $\boldsymbol{q}(\varepsilon . q=0)$ and frequency $\omega=c q$. In (6.7), $\Omega$ is the volume of the large space in which the photon field is quantized. Since this method of quantization is slightly artificial, and would not be required in a consistent field-theoretic treatment, observable quantities must be independent of the quantization method, i.e. independent of $\Omega$.

For the moment, the spin contribution to the interaction $V$ in (6.6) will be neglected, and so $V$ is proportional to the integral over space of $\boldsymbol{J}$. $\boldsymbol{A}$. The matrix 
element of this quantity required in the transition rate is, using $|i\rangle=|0\rangle|\mu\rangle$ and $|f\rangle=|1\rangle\left|\mu^{\prime}\right\rangle$,

$$
\langle f|\mathbf{J} \cdot \mathbf{A}| i\rangle=\left\langle\mu^{\prime}|\mathbf{J}| \mu\right\rangle \cdot\langle 1|\mathbf{A}| 0\rangle
$$

Here, the matrix element of $\boldsymbol{A}$ is (6.7) with $\mathrm{t}=0$, since the time-dependence is treated in the time-dependent perturbation theory leading to Fermi's Golden Rule, eqn (6.6), in which the factor $\exp (\mathrm{i} \omega \mathrm{t})$ is manifest in the delta-function expressing conservation of energy. Assembling expressions for the various quantities in (6.6) the transition rate is,

$$
\left(\frac{4 \pi^{2} \hbar c \alpha}{\Omega \omega}\right) \delta\left(\mathrm{E}_{\mu^{\prime}}+\hbar \omega-\mathrm{E}_{\mu}\right)\left|\left\langle\mu^{\prime}\left|\varepsilon . \mathbf{J}_{\mathbf{4}}\right| \mu\right\rangle\right|^{2}
$$

in which

$$
\mathbf{J}_{\mathbf{q}}=\int \mathrm{d} \mathbf{r} \mathrm{e}^{-i \mathrm{q} \cdot \mathbf{r}} \mathbf{J}(\mathbf{r})
$$

arises from the spatial integral in (6.4) and the factor $\exp (-i q . r)$ in (6.7) for the matrix element of $A$. The total transition rate for spontaneous emission, the observed quantity, is obtained from (6.8) by summing over $q$. For plane-wave states,

$$
\frac{1}{\Omega} \sum_{\mathrm{q}} \rightarrow\left(\frac{1}{2 \pi}\right)^{3} \int \mathrm{d} \mathbf{q} \equiv\left(\frac{1}{2 \pi \mathrm{c}}\right)^{3} \int \omega^{2} d \omega d \hat{\mathbf{q}}
$$

whence the total transition rate for spontaneous emission, calculated to lowest order in the fine structure constant, is

$$
\left(\frac{\hbar \alpha}{2 \pi \mathrm{c}^{2}}\right) \int \mathrm{d} \omega \mathrm{d} \hat{\mathbf{g}} \omega\left|\left\langle\mu^{\prime}\left|\varepsilon \cdot \mathbf{J}_{\mathbf{q}}\right| \mu\right\rangle\right|^{2} \delta\left(\mathrm{E}_{\mu^{\prime}}+\hbar \omega-\mathrm{E}_{\mu}\right) .
$$

From this result it follows that, the transition rate for emission into a solid angle $d \hat{q}$, when the atom changes from a statc labelled $\mu$ to a state labelled $\mu^{\prime}$, is

$$
\Gamma=\left(\frac{\alpha}{2 \pi c^{2}}\right) \omega\left|\left\langle\mu^{\prime}\left|\varepsilon, \mathbf{J}_{\mathrm{q}}\right| \mu\right\rangle\right|^{2},
$$


where $E_{\mu^{\prime}}+$ hw $=E_{\mu}$ from the conservation of energy. The formula (6.12) relates to a specific value for the polarization $\varepsilon$ of the emitted photon.

When the wavelength of the emitted radiation is large compared to the spatial extent of the wave functions that contribute in $|\mu\rangle$ and $\left|\mu^{\prime}\right\rangle$ the matrix element in (6.12) can sensibly be computed as an expansion in $q$. The zeroth-order term in such an expansion,

$$
\begin{aligned}
\int d \mathbf{r}\left\langle\mu^{\prime}|\mathbf{J}(\mathbf{r})| \mu\right\rangle=\frac{1}{\mathrm{~m}^{*}}\left\langle\mu^{\prime}|\mathbf{P}| \mu\right\rangle \\
=(i / \hbar)\left\langle\mu^{\prime}|[\mathcal{H}, \mathbf{R}]| \mu\right\rangle \\
=(i / \hbar)\left(\mathrm{E}_{\mu^{\prime}}-\mathrm{E}_{\mu}\right)\left\langle\mu^{\prime}|\mathbf{R}| \mu\right\rangle,
\end{aligned}
$$

where $\mathbf{P}$ is the total linear momentum and $\left\langle\mu^{\prime}|\boldsymbol{R}| \mu\right\rangle$ is an off-diagonal matrix element of the dipole moment operator. Substituting (6.13) in (6.12) and making use of the conservation of energy, the dipole approximation to the rate of spontaneous emission is

$$
\gamma=\left(\frac{\alpha \omega^{3}}{2 \pi \mathrm{c}^{2}}\right)\left|\left\langle\mu^{\prime}|\varepsilon \cdot \mathbf{R}| \mu\right\rangle\right|^{2} .
$$

To sum over the polarization states of the emitted photon, and integrate over all propagation directions, it is possible to proceed along the same steps used in going from (3.1) to (3.2) in the discussion of the Thomson formula; the result is to introduce a factor $(8 \pi / 3)$ in $(6.14)$.

The electric dipole transition (E1) matrix element in (6.14) might be zero, in which case the next term in the expansion of $\boldsymbol{J}_{\boldsymbol{q}}$ in $\boldsymbol{q}$ is required. This term produces magnetic dipole (M1) and electric quadrupole transitions (E2). Referring to (6.9), the term in question is,

$$
-i \int d \mathbf{r}(\mathbf{q} \cdot \mathbf{r}) \mathbf{J}(\mathbf{r}), \varepsilon
$$

Straightforward vector algebra gives,

$$
(\mathbf{q} \times \varepsilon) \cdot(\mathbf{r} \times J)=(\mathbf{q} \cdot \mathbf{r})(J \cdot \varepsilon)-(\mathbf{q} \cdot J)(\mathbf{r} \cdot \varepsilon),
$$




$$
\begin{aligned}
(\mathbf{q} \cdot \mathbf{r}) & (J \cdot \varepsilon)=\frac{1}{2}(\mathbf{q} \times \varepsilon) \cdot(\mathbf{r} \times J) \\
+ & \frac{1}{2}\{(\mathbf{q} \cdot \mathbf{r})(J \cdot \varepsilon)+(\mathbf{q} \cdot J)(\mathbf{r} \cdot \varepsilon)\}
\end{aligned}
$$

Now, using the definition (6.3) of $J(r)$,

$$
\int d \mathbf{r} \mathbf{r} \times J(\mathbf{r})=\frac{1}{\mathrm{~m}^{*}} \mathbf{L},
$$

where $L$ is the total orbital angular momentum operator. Furthermore, the relation $\boldsymbol{H}=\operatorname{curl} \boldsymbol{A}$, and expression (6.7) for the one-photon matrix element of $\boldsymbol{A}$, shows that $(q \times \varepsilon)$ is related to the plane-wave expansion of the magnetic field. Combining these results reveals that the first term in (6.16), for the interaction (6.15), represents the interaction of the magnetic field of the photon field and the orbital moment of the assembly of charges. To this is added the influence of the spin interaction, and the components combine to produce the total magnetic moment operator $\mu_{B}(L+2 S)$, interacting with the magnetic field.

The remaining terms in (6.16) can be shown to produce electric quadrupole transitions. The $E 2$ and $M 1$ amplitudes are weaker than $E 1$, when it does not vanish. For example, the latter is of order $\omega \mathrm{a}_{0}$, since in (6.17) the matrix element of $\boldsymbol{R}$ for an atom $\sim a_{O}$, while the magnetic amplitude $\sim(\hbar q / m) \equiv(E / m c)$, so the ratio of amplitudes $(M 1 / E 1) \sim \alpha$. The $M 1$ and $E 2$ amplitudes are of a similar size, except, for example, transitions between fine structure components of a given term when $M 1$ radiation is more probable than $E 2$. 


\section{Scattering by Free Charges}

For the most part the discussion here is centred on the non-relativistic charge density response function (5.3) evaluated for free charges. The corresponding crosssection is (4.9). The relativistic result, usually referred to as the Klein-Nishina formula, is discussed at the end of the section. In free space a free electron cannot emit or absorb a photon without violating energy or momentum conservation. Therefore there are no first-order processes involving the $\boldsymbol{p} . \boldsymbol{A}$ terms in the interaction (6.1). However, there are first-order processes involving the $A^{2}$ term, which is quadratic in photon operators. The scattering of photons, which is of interest here, is a process in which one photon is destroyed and another created. The quantum theory of scattering applied to the $A^{2}$ term in (6.1) shows that, within the first Born approximation (equivalent to the use of Fermi's Golden Rule for (ransition rates), eqns (4.9) and (5.3) apply.

Perhaps the most direct way to calculate $S(k, \omega)$ for a system of charge carriers described by quantum mechanics is to employ a second quantized representation for $n(k)$. If the carriers obey Fermi statistics, correct for spin $-1 / 2$ particles, and the annihilation and creation operators are denoted by $c, c^{+}$, respectively,

$$
\mathrm{n}(\mathrm{k})=\sum_{p \sigma} \mathrm{c}^{+}{ }_{k+p \sigma} \mathrm{c}_{p \sigma},
$$

where $p$ and $\sigma$ are wave vector and spin labels. To form $n(k, t)$, note that for free particles,

$$
\mathrm{c}_{\mathrm{p} \sigma}^{+}(\mathrm{t})=\exp \left(i \mathrm{t} / \mathrm{p} \mathrm{p}^{2} / 2 \mathrm{~m} *\right) \mathrm{c}_{\mathrm{p} \sigma}^{+}
$$

Hence, for $k \neq 0$,

$$
\left\langle n^{+}(\mathbf{k}) n(k, t)\right\rangle=\sum_{p \sigma} f_{p}\left(1-f_{k+p}\right) \exp \left\{\frac{i v / z}{2 m^{*}}\left(k^{2}+2 k \cdot p\right)\right\},
$$

where $f_{p}$ is the Fermi distribution function,

$$
f_{\mathrm{p}}=\left(\exp \left\{\beta\left(\mathrm{E}_{\mathrm{p}}-\mu\right)\right\}+1\right)^{-1}
$$


and the chemical potential $\mu$ is determined, in the usual way, from the number of carriers. Note that since the one-particle energy $\mathrm{E}_{\mathrm{p}}=(\hbar \mathrm{p})^{2} / 2 \mathrm{~m} *$ is independent of the spin label, the sum on $\sigma$ in (7.3) merely produces a factor 2. From (5.3) and (7.3),

$$
\begin{aligned}
& S(\mathbf{k}, \omega)=(2 / N) \sum_{p} \delta\left\{\hbar \omega+E_{p}-E_{k+p}\right\} f_{p}\left(1-f_{k+p}\right) \\
& =\{1+n(\omega)\}(2 / N) \sum_{p} \delta\left\{\hbar \omega+E_{p}-E_{k+p}\right\}\left(f_{p}-f_{k+p}\right),
\end{aligned}
$$

and in the second form of the result the first factor is defined by $n(\omega)=\{\exp (\hbar \omega \beta)-1\}^{-1}$. The Boltzmann limit is recovered from (7.5) when the chemical potential satisfies $\exp (\mu \beta)<<1$, and in this case it has the value,

$$
\exp (\mu \beta)=\frac{\mathrm{n}_{\mathrm{o}}}{2}\left(2 \pi \hbar^{2} \beta / \mathrm{m} *\right)^{3 / 2} \ll \mathrm{I},
$$

which is a condition on particle density $n_{O}$, temperature and mass.

Another interesting limiting case of (7.5) is when the scattering vector $k$ is large. For sufficiently large $k, f_{\mathrm{p}} \gg f_{k+p}$, whence $S(k, \omega)$ approaches the value

$$
\mathrm{S}(\mathbf{k}, \omega) \cong\{1+\mathrm{n}(\omega)\}\left(\frac{2}{\mathrm{~N}}\right) \sum_{\mathrm{p}} \delta\left\{\hbar \omega+\mathrm{E}_{\mathrm{p}}-\mathrm{E}_{\mathbf{k}+\mathrm{p}}\right\} f_{\mathrm{p}} .
$$

which describes the Compton process discussed in $\$ 1$. The structure of the right-hand side is a delta-function, expressing conservation of energy for free particles, weighted by $f_{\mathbf{p}}$ which is the momentum distribution function for free particles.

As the final topic in this section, let us turn to the Klein-Nishina formula for relativistic scattering a photon by a liee electron that is at rest before the collision (Berestetskii et al. 1982). The latter is polarized, and the average value of the spin is $\langle s\rangle$. The primary radiation is assumed to contain linear and circular polarization, represented by Stokes parameters $P_{2}$ and $P_{3}$. The values $P_{3}=+1$ and $P_{3}=-1$ correspond to complete linear polarization and, respectively, labelled $\sigma$ - and $\pi$ - polarization states by many authors. The parameter $P_{2}$ represents the degree of circular polarization; with our 
convention, the probability that the primary photon has right-hand or left-hand circular polarization is respectively $\left(1+P_{2}\right) / 2$ and $\left(1-P_{2}\right) / 2$. In scattering, the primary photon is deflected through an angle $\theta$, and the relativistic relation between photon energy change and the scattering angle is,

$$
\frac{1}{q^{\prime}}-\frac{1}{q}=(\hbar / \mathrm{mc})(1-\cos \theta) .
$$

Having dispatched these necessary definitions, the exact cross-section is,

$$
\frac{d \sigma}{d \Omega}=\frac{1}{2} \mathrm{re}^{2}\left(\frac{\mathrm{q}^{\prime}}{\mathrm{q}}\right)^{2}\left\{\frac{\mathrm{q}}{\mathrm{q}^{\prime}}+\frac{\mathrm{q}^{\prime}}{\mathrm{q}}+\left(\mathrm{P}_{3}-1\right) \sin ^{2} \theta-2 \tau \mathrm{P}_{2}(1-\cos \theta)\langle\mathrm{s}\rangle \cdot\left(\hat{\mathrm{q}} \cos \theta+\mathrm{q}^{\prime} / \mathrm{q}\right)\right\} .
$$

Here, the dimensionless quantily $\tau=(\hbar g / m c)$. The contribution from the electron spin vanishes if $\langle s\rangle$ is perpendicular to the scattering plane, delined by $q$ and $q^{\prime}$. When this condition holds, the cross-section is that for scaltering by unpolarized electrons; photons polarized perpendicular to the scattering plane $\left(P_{3}=1\right)$ have a larger cross-section than for photons in the plane $\left(P_{3}=-1\right)$. To engage in scattering the spin of the electron must have a projection on the scattering plane, as already mentioned, and the primary photon must be circularly polarized $\left(P_{2} \neq 0\right)$. The spin-dependent term can be thought of as an interference between charge and magnetic (spin) scattering induced by circular polarization. There is no orbital angular momentum for a free electron, of course.

The classical non-relativistic result is obtained for $\hbar q<<m c$, i.e. $\tau<<1$. In this instance $q^{\prime} \sim q$, and the cross-section reduces to

$$
\frac{\mathrm{d} \sigma}{\mathrm{d} \Omega}=\mathrm{re}_{\mathrm{e}}^{2}\left\{1+\frac{1}{2}\left(P_{3}-1\right) \sin ^{2} \theta-\tau \mathrm{P}_{2}(1-\cos \theta)\langle\mathrm{s}\rangle \cdot\left(\hat{\mathbf{q}} \cos \theta+\hat{\mathbf{q}}^{\prime}\right)\right\} .
$$

For $P_{2}=P_{3}=0,(7.10)$ is the same as $(3.2)$ for one electron $(N=1)$, i.e. Thomson's formula is recovered, as it should be. 


\section{Compton Limit}

The Compton limit of scattering is illustrated in terms of photon scattering by charged particles for which the response function is (5.3). The treatment is non-relativistic. No assumption is made about the statistics used to describe the particles in the target assembly. In fact, the results apply to photon scattering from charge carriers, and neutron scattering from nuclei which might be described by Bose or Fermi statistics. The essential conditions are that the primary energy is large compared with the binding energy of a particle, and the scattering vector is large, so $(a k) \gg>1$ where a is the mean separation between particles. The first condition permits the use of a short-time approximation for the correlation function, on the grounds that the collision is almost instantaneous, and $(a k) \gg 1$ means that the incoherent approximation to scattering is reasonable.

With the definition $E_{k}=(\mathrm{h} k)^{2} / 2 m^{*}$, the short-time approximation gives,

$$
\left\langle n^{+}(\mathbf{k}) n(\mathbf{k}, t)\right\rangle \cong \sum_{j j} \exp \left(\frac{i t}{h} E_{k} \delta_{j j^{\prime}}\right)\left\langle\exp \left\{i k \cdot\left(\mathbf{R}_{j}-\mathbf{R}_{j^{\prime}}\right)+\frac{i t}{m^{*}} \mathbf{k} \cdot \mathbf{p}_{j}\right\}\right\rangle .
$$

where $\mathbf{p}$ is the momentum operator conjugate to $\mathbf{R}$. Note that the recoil energy enters for just the self-terms in the sum, i.e. $j=j^{\prime}$. A derivation of (8.1) given by Lovesey (1987b) is outlined in $\$ 13.3 .3$.

Next, the incoherent approximation, discussed in $\$ 2$, is invoked. It amounts to retaining the self-terms in (8.1), on the grounds that for $(a k) \gg 1$ the terms $j \neq j$ are negligible because of the rapid variation in the phase factors. The expression,

$$
\left\langle\mathrm{n}^{+}(\mathbf{k}) \mathrm{n}(\mathbf{k}, \mathrm{t})\right\rangle \cong \sum_{\mathrm{j}} \exp \left(\frac{\mathrm{it}}{h} \mathrm{E}_{\mathrm{k}}\right)\left\langle\exp \left(\frac{\mathrm{it}}{\mathrm{m}^{*}} \mathbf{k} \cdot \mathbf{p}_{\mathrm{j}}\right)\right\rangle
$$

is the value of the density auto-correlation function in the Compton limit; the right-hand side of (8.2) is often referred to as the impulse approximation.

To bring (8.2) to a more useful form, let us introduce the momentum distribution function

$$
\mathrm{N} \rho(\mathbf{q})=\left\langle\sum_{j} \delta\left(\hbar \mathbf{q}-\mathbf{p}_{\mathbf{j}}\right)\right\rangle
$$


where $N$ is the number of particles. Note that the dimension of $\rho(q)$ is $(1 / \text { momentum })^{3}$, and

$$
\int d \mathbf{q} \rho(\mathbf{q})=1 / \hbar^{3}
$$

To make use of $\rho(q)$ in (8.2) insert unity in the guise of

$$
\hbar^{3} \int d \mathbf{q} \delta\left(\hbar \mathbf{q}-\mathbf{p}_{\mathrm{j}}\right)=1
$$

to obtain for $(8.2)$,

$$
\langle\mathrm{n}+(\mathbf{k}) \mathrm{n}(\mathbf{k}, \mathrm{t})) \cong \mathrm{N} \hbar^{3} \int \mathrm{dq} \rho(\mathbf{q}) \exp \left(\frac{\mathrm{it}}{\hbar} \mathrm{E}_{\mathrm{k}}+\frac{\mathrm{ith}}{\mathrm{m}^{*}} \mathbf{k} \cdot \mathbf{q}\right) .
$$

The corresponding result for $S(k, \omega)$, the Compton limit of the charge response function, is

$$
\mathrm{S}(\mathbf{k}, \omega)=\hbar^{3} \int \mathrm{d} \mathbf{q} \rho(\mathbf{q}) \delta\left\{\hbar \omega-\mathrm{E}_{\mathbf{k}}-\hbar^{2} \mathbf{k} \cdot \mathbf{q} / \mathrm{m} *\right\} \text {, }
$$

which is in complete accord with (7.7). Note that the static structure factor associated with this expression is

$$
\hbar \int_{-\infty}^{\infty} \mathrm{d} \omega \mathrm{S}(\mathbf{k}, \omega)=1
$$

As expected, this agrees with the lormula for Thomson scattering evaluated in the incoherent approximation.

An alternative way forward from $(8.2)$ is to introduce the momentum representation of the electron wave functions. Let

$$
\Psi_{\mu}(\mathbf{R})=(1 / 2 \pi)^{3 / 2} \int \mathrm{d} \boldsymbol{q} \exp (\text { iq. } \mathbf{R}) \chi_{\mu}(\boldsymbol{q})
$$

and form the diagonal matrix element of the exponential operator in (8.2), 


$$
\int \mathrm{d} \mathbf{R} \Psi_{\mu}^{*}(\mathbf{R}) \exp \left(\text { itk. p/m*) } \Psi_{\mu}(\mathbf{R})=\int \mathrm{d} \mathbf{q}\left|\chi_{\mu}(\mathbf{q})\right|^{2} \exp \left(\mathrm{i} \hbar \mathrm{t} \mathbf{k} \cdot \mathbf{q} / \mathrm{m}^{*}\right)\right.
$$

The last result leads immediately to an expression of the form (8.5) when $\rho(q)$ is identified with the average value of $\left|\chi_{\mu}\right|^{2}$ 


\section{Generalized Kramers - Heisenberg Formula}

The results discussed here have applications in the interpretation of resonant and nonresonant events. Effects due to the spin of an electron are not included, although it is not very difficult to do so (Sakurai, 1987). Instead, we delay our discussion of spin-dependent events to $\$ 11$ where our most general expression for the scattering amplitude is recorded. One reason for giving here a discussion of a less general result for the amplitude is that it is possible to get an appreciation for the kcy idcas and approximations while pursuing an algebraically simpler problem which none the less is of use in the interpretation of experiments. In particular, the generalized Kramers-Heisenberg formula provides a basis for the contribution from orbital magnetism to the diffraction cross-section, and the interpretation of dichroism and elastic resonant scaltering from magnetic materials. It is also the basis for the interpretation of resonant Raman scattering which is potentially well suited to studies of magnetic materials using circularly polarized radiation (Eteläniemi et al,, 1992).

The interaction between a photon and an assembly of charged particles is discussed in $\S 6$, where it is shown that the interaction $V$ is a sum of two terms of the form $p . A$ and $A^{2}$. The vector potential $\boldsymbol{A}$ is a linear combination of photon annihilation and creation operators, so the $\boldsymbol{p} . \boldsymbol{A}$ term is linear and the $A^{2}$ term is quadratic in photon operators, and we shall employ the suggestive notation,

$$
V=V_{1}+V_{2}
$$

As mentioned in $\$ 6$, there is in addition to the terms just discussed a spin-dependent interaction. The term provided in $\$ 6$ is linear in $A$, since it is just $s . H$ and $H=\operatorname{curl} A$, and can be added to $V_{1}$. There is also a spin-dependent term of order $A^{2}$, to be added to $V_{2}$, generated by the spin-orbit coupling. But in the present discussion we set aside all the spindependent terms, and work with only the p.A $\left(\equiv V_{1}\right)$ and $A^{2}\left(\equiv V_{2}\right)$ terms. This decision is taken to keep the present discussion simple. However, spin-dependent scattering for a polarized free electron system, logether with the Klein-Nishina formula, is discussed in $\$ 7$.

Photon scattering consists of the absorption of a primary photon and the simultaneous emission of a secondary pholon. The wave vectors of the primary and secondary photons are $\boldsymbol{q}$ and $\boldsymbol{q}^{\prime}$, and the scattering vector,

$$
k=q-q^{\prime}
$$

The concomitant change in energy is, 
The concomitant change in energy is,

$$
\hbar \omega=\hbar c\left(q-q^{\prime}\right) \equiv E-E^{\prime} .
$$

The polarizations vectors are $\varepsilon$ and $\varepsilon^{\prime}$, and $\varepsilon \cdot q=\varepsilon^{\prime} \cdot q^{\prime}=0$. For non-resonant processes the differential cross-section is a function of four variables, $k$ and $\omega$.

Let us illustrate the steps involved in calculating the non-relativistic cross-section by considering the contribution from the term,

$$
V_{2}=\frac{\mathrm{e}^{2}}{2 \mathrm{~m}^{*} \mathrm{c}^{2}} \int d \mathbf{r} \rho_{\mathrm{o}}(\mathbf{r}) \mathrm{A}^{2}(\mathbf{r})
$$

where $\rho_{o}$ is the single particle distribution function (2.7) and $m^{*}$ is the effective mass. The initial state of the composite system, comprised by the primary photon and target assembly, is described by the state function $\{|q, \varepsilon\rangle|\mu\rangle\}$. The final state is described by a similar function with the labels replaced by primed labels.

The cross-section is calculated with Fermi's Golden Rule for transition rates. In the present case the rate is,

$$
\Gamma=\frac{2 \pi}{\hbar}\left|\left\{\left\langle\mathbf{q}^{\prime},\left.\boldsymbol{\varepsilon}^{\prime}\left|\left\langle\mu^{\prime}\right|\right\} \mathrm{V}_{2}\{|\mu\rangle|\mathbf{q}, \boldsymbol{\varepsilon}\rangle\}\right|^{2}\right.\right.\right.
$$

To get the cross-section from $\Gamma$ it is necessary to multiply by the number of allowed states in an energy interval between $E^{\prime}$ and $\left(E^{\prime}+d E^{\prime}\right)$, and divide by flux density. The differential cross-section is found to be,

$$
\frac{\mathrm{d} \sigma}{\mathrm{d} \Omega}=\left(\frac{\mathrm{mr}_{\mathrm{e}}}{\mathrm{m}^{*}}\right)^{2}\left(\frac{\mathrm{q}^{\prime}}{\mathrm{q}}\right)\left\{\boldsymbol{\varepsilon} \cdot \boldsymbol{\varepsilon}^{\prime}\right\}^{2}\left|\left\langle\mu^{\prime}|\mathrm{n}(\mathbf{k})| \mu\right\rangle\right|^{2}
$$

in which energy conservation is provided by $\hbar \omega=E_{\mu^{\prime}}-E_{\mu}$. The expression (9.6) is the basis for eqn (4.5) for the partial differential cross-section for photon scattering by an asembly of identical charges with mass $m^{*}$.

The scattering process which yields the differential cross-section (9.6) can be expressed in terms of a space-time Feynman diagram depicted in Fig. (6). Here, charge 
diagram illustrates that there is no internal structure to the interaction vertex in the lowestorder $A^{2}$ contribution to the scattering process. Since the interaction $V_{1}$ is linear in photon operators it must operate twice to generate scatlering. Hence, the first-order contribution to a scattering process from $V_{1}$ occurs in second-order perturbation theory. The structure of conventional perturbation theory in quantum mechanics reveals that a second-order process contains intermediate states, so the vertex in the Feynman diagram contains structure, as depicted in Fig. (6). There seems litle to be gained from a detailed derivation of the contribution from $V_{1}$ to the differential cross-section, so we move immediately to the final expression.

To provide a compact expression it is prudent to define a momentum density,

$$
J(\mathbf{q})=\sum_{j} \mathbf{p}_{\mathrm{j}} \exp \left(\mathrm{iq} \cdot \mathbf{R}_{\mathrm{j}}\right)
$$

where the sum is over all the charged particles in the assembly, and $\boldsymbol{p}$ and $\boldsymbol{R}$ are conjugate variables. Note that the Hermitian conjugate,

$$
J^{+}(\mathbf{q})=\sum_{j} \exp \left(-i \mathbf{q} \cdot \mathbf{R}_{j}\right) \mathbf{p}_{j}=\sum_{j}\left(\mathbf{p}_{j}+\hbar \mathbf{q}\right) \exp \left(-i \mathbf{q} \cdot \mathbf{R}_{j}\right) \equiv J(-\mathbf{q})+\hbar \mathbf{q} n^{+}(\mathbf{q})
$$

so $\varepsilon . J^{*}(q)=\varepsilon . J(-q)$. With this notation, a dimensionless scattering amplitude operator for processes generated by $V_{1}$ and $V_{2}$ is,

$$
\begin{aligned}
\mathrm{G}=\boldsymbol{\varepsilon} \cdot \varepsilon^{\prime} & \mathrm{n}(\mathbf{k})+\left(\frac{1}{\mathrm{~m}^{*}}\right)\left\{\boldsymbol{\varepsilon} \cdot \mathbf{J}(\mathbf{q})\left(\mathrm{E}_{\mu}-\mathrm{E}^{\prime}-\mathscr{H}\right)^{-1} \varepsilon^{\prime} \cdot \mathbf{J}^{+}\left(\mathbf{q}^{\prime}\right)\right. \\
& \left.+\varepsilon^{\prime} \cdot \mathbf{J}^{+}\left(\mathbf{q}^{\prime}\right)\left(\mathrm{E}_{\mu}+\mathrm{E}-\mathfrak{l}\right)^{-1} \varepsilon \cdot \mathbf{J}(\mathbf{q})\right\}
\end{aligned}
$$

where the Hamiltonian $\mathscr{H}$ describes the target assembly, $E_{\mu}$ is the energy of the initial target state $\left(\mathscr{H}|\mu\rangle=\mathrm{E}_{\mu}|\mu\rangle\right)$ and $E=\hbar i c q, E^{\prime}=\hbar c q$. The resolvent operators $\left(E_{\mu}-E^{\prime}-\mathscr{H}^{\prime-1}\right.$ and $\left(E_{\mu}+E-\mathscr{H}^{-1}\right.$ are delined with an inlinilesimal negative imaginary part added to $\mathscr{K}$ (not shown explicitly) for pole avoidance. The avoidance rule is important when the poles of $(9.9)$ with respect to the energies of intermediate states are in the region of the continuous spectrum; e.g. if the initial state is the ground state of an atom this would occur for $E$ 
exceeding the ionization threshold of the atom, whereas in a molecule the threshold for dissociation into atoms takes the place of the ionization threshold.

With the result (9.9) for $G$, the differential cross-section is found to be,

$$
\frac{\mathrm{d} \sigma}{\mathrm{d} \Omega}=\left(\frac{\mathrm{mr}_{\mathrm{e}}}{\mathrm{m}^{*}}\right)^{2}\left(\frac{\mathrm{q}^{\prime}}{\mathrm{q}}\right)\left|\left\langle\mu^{\prime}|\mathrm{G}| \mu\right\rangle\right|^{2} .
$$

This expression does not contain effects due to the spin magnetic moment of the electrons, as discussed earlier in this section. But it does contain magnetic scattering in the form of a contribution that can be identified as duc 10 orbital angular momentum. This might be expected in view of the existence of orbital angular momentum displayed in $M 1$ transition, and discussed in $\$ 6$. The occurence of orbital angular momentum in (9.10) is taken up in the following section.

To conclude this section, let us consider the explicit form of one of the two 'resonance-type' contributions in $G$, coming lrom $V_{1}$ in second-order. The infinitesimal negative imaginary part added to $\mathscr{L}$ is denoted by $(-i \gamma / 2)$. The choice of notation is meant to convey the notion that physically this contribution arises from damping, just like we encountered in the purely classical theory of scattering reviewed in $\$ 1.2$. Selecting to examine the third term in (9.9), and inserting a complete set of states for $\mathscr{H}$ labelled by quantum numbers $\{\bar{\mu}\}$, including all states from the discrete and continuous parts of the spectrum, one finds

$$
\left(\frac{1}{m^{*}}\right) \sum_{\bar{\mu}} \frac{\varepsilon^{\prime} . J^{+}\left(q^{\prime}\right)|\bar{\mu}\rangle\langle\bar{\mu}| \varepsilon \cdot \mathbf{J}(\mathbf{q})}{\left(E_{\mu}+E-E_{\bar{\mu}}+i \gamma_{\bar{\mu}} / 2\right)} .
$$

In practice $\gamma_{\bar{\mu}}$ can be neglected except when $E \cong E_{\bar{\mu}}-E_{\mu}$.

In general, the resonant amplitude is much larger than the sum of non-resonant amplitudes. This arises because the magnitude of the resonant amplitude, estimated in $\$ 1.1$, is of the order $\lambda$ while the magnitude of the non-resonant amplitude if of the order of $r_{e}$. If the condition for resonance with a particular intermediate state is almost satisfied, so $E \cong E_{\bar{\mu}}-E_{\mu}$, and the intermediate state is nondegencrate, the single-level resonance formula is obtained immediately from $(9.10)$ and $(9.11)$, namely, 


$$
\frac{\mathrm{d} \sigma}{\mathrm{d} \Omega}=\left(\frac{\mathrm{mr} \mathrm{e}}{\mathrm{m}^{*}}\right)^{2}\left(\frac{\mathrm{q}^{\prime}}{\mathrm{q}}\right)\left(\frac{1}{\mathrm{~m}^{*}}\right)^{2} \frac{\left|\left\langle\mu^{\prime}\left|\varepsilon^{\prime} \cdot \mathbf{J}+\left(\mathbf{q}^{\prime}\right)\right| \bar{\mu}\right\rangle\langle\bar{\mu}|\varepsilon \cdot \mathbf{J}(\mathbf{q})| \mu\rangle\right|^{2}}{\left(\mathrm{E}_{\mu}+\mathrm{E}-\mathrm{E}_{\bar{\mu}}\right)^{2}+\left(\gamma_{\bar{\mu}} / 2\right)^{2}} .
$$

If $q$ and $q^{\prime}$ are small the matrix elements which appear in (9.12) can be calculated for $J(0)=\boldsymbol{P}$ where $\boldsymbol{P}$ is the total momentum. Formula (6.13) yields the result,

$$
\left\langle\mu^{\prime}|\mathbf{P}| \bar{\mu}\right\rangle=\left(\mathrm{im}^{*} / \hbar\right)\left(\mathrm{E}_{\mu^{\prime}}-\mathrm{E}_{\overline{\boldsymbol{\mu}}}\right)\left\langle\mu^{\prime}|\mathbf{R}| \bar{\mu}\right\rangle,
$$

in which $\left\langle\mu^{\prime}|\boldsymbol{R}| \bar{\mu}\right\rangle$ is an off-diagonal matrix element of the dipole moment operator. The next term in an expansion of $\boldsymbol{J}(\boldsymbol{q})$ in $\boldsymbol{q}$ produces, as demonstrated in $\S 6$, the total orbital angular momentum operator interacting with a component of $\boldsymbol{H}=\operatorname{curl} \boldsymbol{A}$, and the quadrupole operator. Hence, beyond the dipole approximation, resonant scattering contains processes that are explicitly magnetic in character. 


\section{Scattering by Orbital Magnetism}

The object of the present discussion is to expose the orbital magnetic moment in the scattering amplitude operator (9.9), and demonstrate its simple relation to the appropriate operator in the amplitude for magnetic neutron scattering. This identification has an immediate practical benefit since it enables us to utilize the knowledge of the properties of the operator in the literature on neutron scattering. In fact, the non-resonant limit of $\mathrm{G}$ is shown to contain the operator $\mathbf{Z}$ defined by (3.19) which in turn is related to $\mathbf{T}$ that describe scattering by orbital magnetism. The experimental evidence, discussed in $\$ \S 12$ and 13 , is that orbital magnetism is observed in Bragg diffraction but not in Compton scattering. The latter observation is supported by a calculation described in $\$ 13.3 .2$ for a specific model of a Compton scattering event. Matrix elements of $\mathbf{T}(\mathrm{k})$ for atomic $3 \mathrm{~d}$ and $4 \mathrm{f}$ states of a single multiplet are listed by Balcar and Lovesey (1989).

Turning to the definition of the photon scattering amplitude operator (9.9), it is evident that the operator $T(k)$ defined in (3.20) can arise only from the last two terms, generated by $V_{1}$ taken in second-order. When $E, E^{\prime}$ are much in excess of the energies of states in the spectrum of $\mathscr{H}$, the Hamiltonian that describes the target assembly, the resolvent operators in $G$ can be salely expanded in $(1 / E)$ and $\left(1 / E^{\prime}\right)$. Keeping the first-order terms in such an expansion leads to a non-resonant scattering amplitude operator,

$$
\mathrm{G}=\boldsymbol{\varepsilon} \cdot \boldsymbol{\varepsilon}^{\prime} \mathrm{n}(\mathbf{k})+\left(\frac{1}{\mathrm{~m}^{*}}\right)\left\{\left(\frac{1}{E}\right) \varepsilon^{\prime} \cdot \mathbf{J}^{+}\left(\mathbf{q}^{\prime}\right) \boldsymbol{\varepsilon} \cdot \mathbf{J}(\mathbf{q})-\left(\frac{1}{E^{\prime}}\right) \varepsilon \cdot \mathbf{J}(\mathbf{q}) \varepsilon^{\prime} \cdot \mathbf{J}^{+}\left(\mathbf{q}^{\prime}\right)\right\} .
$$

Since $\boldsymbol{J}(q)$ is the spatial Fourier transform of the linear momentum, eqn (9.7), the second two terms are manifestly quadratic in the momenta of the particles. But, because $p_{j}$ and $\boldsymbol{R}_{j}$ do not commute there is, in fact, a $t \mathrm{crm}$ linear in the momenta and proportional to $k$;

$$
\begin{aligned}
& \varepsilon^{\prime} \cdot \mathbf{J}^{+}\left(\mathbf{q}^{\prime}\right) \boldsymbol{\varepsilon} \cdot \mathbf{J}(\mathbf{q})=\sum_{\mathrm{j} j^{\prime}} \mathrm{e}^{-\mathrm{i} \boldsymbol{q}^{\prime} \cdot \boldsymbol{R}_{\mathrm{j}}} \boldsymbol{\varepsilon}^{\prime} \cdot \mathbf{p}_{\mathrm{j}} \mathrm{e}^{\mathrm{iq} \cdot \boldsymbol{R}_{j}} \boldsymbol{\varepsilon} \cdot \mathbf{p}_{\mathrm{j}}
\end{aligned}
$$

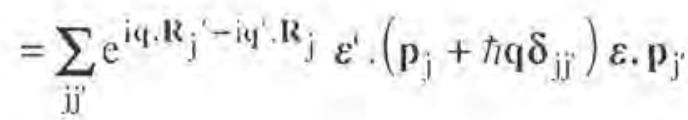

in which $q$ can be replaced by $k$ because $\varepsilon^{\prime} \cdot q^{\prime}=0$. In the subsequent development, terms quadratic in $p$ are dropped in favour of linear terms since the latter are the most significant. Two features of the final result contribute to this decision. First, the terms quadratic in $p$ will 
generate smaller effects than those from terms linear in $p$, given that, as we shall see, the latter produce a finite result. The argument here is akin to that which leads to allowed electric dipole transitions dominating magnetic and higher-order electric transitions. Secondly, the quadratic terms assembled in $G$ arise with a factor $\left(E-E^{\prime}\right) / E E^{\prime}$ which vanishes for elastic scattering (Bragg and the static limit) and is small for mildly inelastic, Comptontype, scattering processes (terms quadratic in $\mathbf{p}$ are analysed by Grotch et al. 1983). Hence, in future we shall use,

$$
\varepsilon^{\prime} \cdot \mathbf{J}^{+}\left(\mathbf{q}^{\prime}\right) \varepsilon \cdot \mathbf{J}(\mathbf{q})=\hbar \sum_{\mathrm{j}} \mathrm{e}^{i k \cdot k_{j}}\left(\varepsilon^{\prime} \cdot \mathbf{k}\right)\left(\varepsilon \cdot \mathbf{p}_{\mathrm{j}}\right)
$$

where $k=q-q^{\prime}$, as elsewhere in this text. On inserting this value, and the corresponding value for the last term in (10.1), back into (10.1) the latter becomes,

$$
\mathrm{G}=\boldsymbol{\varepsilon} \cdot \varepsilon^{\prime} \mathrm{n}(\mathbf{k})-\mathrm{i} \tau\left(\frac{\mathrm{m}}{\mathrm{m}^{*}}\right) \varepsilon^{\prime} \times \varepsilon, \mathbf{Z}(\mathbf{k}) .
$$

Here, $\tau=(\hbar q / m c)$ and the condition which applies $10(7.10)$ and results in this section is $\tau \ll 1$. The operator $\mathbf{Z}$ is defined in $\$ 3.3$ logether with its relation to the operator $\mathbf{T}$ used in the interpretation of neutron scattering from orbital magnetism (Balcar and Lovesey 1989). 


\section{Scattering by Spin Magnetism}

The amplitude for spin-dependent scattering of photons by bound electrons has been considered in great detail by Bhatt et al. (1983) and Grotch et al. (1983), to whom the reader is referred for details. The expression quoted here is derived in the cited papers by a systematic treatment of the quantum electrodynamic Hamiltonian for a bound electron. Later in the section we indicate how the result can be derived by addition of spin-dependent terms to the non-relativistic interaction (Sakurai, 1987). While such a scheme is quite similar to the workings reported by Grotch et al. it is more difficult to be confident about making an internally consistent calculation. We find to order $\tau=(\hbar / q / m c)$ that results from the two approaches are identical. Hence, to this level of approximation it is not necessary to employ the mathematical apparatus used by Grotch et al., but their scheme is a safe route by which to obtain higher-order terms, containing products of spin and momentum operators for example.

Following on the development provided in $\$ 9$, we employ a scattering amplitude operator $G$ in terms of which the differential cross-section is $\left(m=m^{*}\right)$,

$$
\frac{d \sigma}{d \Omega}=r_{e}^{2}\left(\frac{q^{\prime}}{q}\right)\left|\left\langle\mu^{\prime}|G| \mu\right\rangle\right|^{2}
$$

Here, $\mu$ and $\mu^{\prime}$ label the initial and final states of the target assembly, and $\hbar c q$ and $\hbar c q^{\prime}$ are the energies of the primary and secondary photons. Polarization of the primary beam is accounted for in (3.14) in terms of a density matrix, and the result (3.16) explicitly displays all possible polarization-induced processes.

In place of the momentum density (9.7) we need,

$$
\mathbf{J}(\mathbf{q})=\sum_{\mathrm{j}}\left(\mathbf{p}_{\mathrm{j}}+\mathrm{i} \hbar_{\mathrm{i}} \mathbf{s}_{\mathrm{j}} \times \mathbf{q}\right) \exp \left(\mathrm{iq} \cdot \mathbf{R}_{\mathrm{j}}\right)
$$

for which

$$
\{\mathbf{J}(\mathbf{q}) \cdot \varepsilon\}^{+}=\mathbf{J}(-\mathbf{q}) \cdot \varepsilon
$$

where $\varepsilon$ is the polarization vector for the primary photon, $\varepsilon \cdot q=0$. In (11.2), $s_{j}$ is the spin operator of the electron labclled by $j$. 
The operator $G$ calculated to first-order in $\tau$ is obtained from eqn (2) in Grotch et al. (1983). Expressed in our notation,

$$
\begin{aligned}
\mathrm{G}=\boldsymbol{\varepsilon} \cdot \boldsymbol{\varepsilon}^{\prime} \mathrm{n}(\mathbf{k})+\frac{i}{2} \tau\left(1+\frac{\mathrm{q}^{\prime}}{\mathrm{q}}\right) \mathrm{S}(\mathbf{k}) \cdot \boldsymbol{\varepsilon} \times \boldsymbol{\varepsilon}^{\prime}+(1 / \mathrm{m})\left\{\boldsymbol{\varepsilon}^{\prime} \cdot \mathbf{J}\left(-\mathbf{q}^{\prime}\right)\left(\mathrm{E}_{\mu}+\mathrm{E}-\mathcal{H}\right)^{-1} \boldsymbol{\varepsilon} \cdot \mathbf{J}(\mathbf{q})\right. \\
\left.\quad+\boldsymbol{\varepsilon} \cdot \mathbf{J}(\mathbf{q})\left(\mathrm{E}_{\mu}-\mathrm{E}^{\prime}-\mathfrak{H}\right)^{-1} \varepsilon^{\prime} \cdot \mathbf{J}\left(-\mathbf{q}^{\prime}\right)\right\} .
\end{aligned}
$$

Here, $n(k)$ is the spatial Fourier transform of the charge density, and $S(k)$ is the spatial Fourier transform of the spin density defined in (2.10) and (3.18), respectively. Examination of (11.4) shows that spin arises in the resonant and non-resonant events, and the structure of (11.4) is similar to (9.9) - on selting $\mathrm{m}=\mathrm{m}^{*}$ - apart from the second contribution which is due solely to the spin density.

The evidence to date is that the results (11.1) and (11.4) are cornerstones for the interpretation of photon scattering by magnetic materials.

Before embarking on further discussions of these important results, part of which is anticipated in $\$ 3$, we will indicate the origins of the spin contributions to the scattering amplitude, which are the features that distinguish (11.4) from expression (9.9) used to derive the orbital contribution. A spin subject to a magnetic field $\boldsymbol{H}$ experiences an interaction $\boldsymbol{s} . \boldsymbol{H}$. In the case of a photon $H=c u r l A$ where $A$ is the vector potential. This interaction is linear in $\boldsymbol{A}$, and hence linear in photon operators, and as such in the calculation of $G$ it is added to the $\boldsymbol{p} . \boldsymbol{A}$ terms and treated in second-order perturbation theory. This is the origin of the spin contribution to the momentum density $(11.2)$. The other spin contribution to $G$ can be viewed as an addition to the charge term generated by the $A^{2}$ interaction, i.e. the seagull diagram in Fig. (6). Such a spin term arises from the spin-orbit interaction which takes the form s. $\dot{\mathbf{A}} \times \mathbf{A}$ where $\dot{A}$ is the time derivative of $\boldsymbol{A}$, and the electric field $\boldsymbol{E} \propto \dot{\boldsymbol{A}}$.

Prior to the papers by Bhatt et al. (1983) and Grotch et al. (1983) several other groups of authors published careful studies of photon scattering by particles of spin $1 / 2$. Notable among these are findings by Gell-Mann and Goldberger (1954) and deBergevin and Brunel (1981). The latter work contains the lirst published account of the orbital contribution to scattering from magnetic materials and its direct relation with the appropriate operator in magnetic neutron scattering. Expressions provided here amount to generalizations of the amplitudes published by deBergevin and Bruncl. Chief among the generalizations is the addition of inelastic terms (deBergevin and Bruncl examined Bragg scattering). 
The scattering amplitude operator $G$ in (11.4) enables us to discuss resonant processes and nonresonant processes. For the latter, resolvent operators are expanded in $(1 / E)$ and $\left({ }^{1} / E^{\prime}\right)$. The expansion is justified when $E, E^{\prime}$ are much in excess of the energies of states in the spectrum of $\mathscr{K}$, the Hamiltonian which describes the charge carriers in the target assembly.

On keeping the leading term in such an expansion, we find

$$
\mathrm{G}=\boldsymbol{\varepsilon} \cdot \varepsilon^{\prime} \mathrm{n}(\mathbf{k})-\mathrm{i} \tau\left\{\mathrm{S}(\mathbf{k}) \cdot \mathbf{B}+\varepsilon^{\prime} \times \varepsilon \cdot \mathbf{Z}(\mathbf{k})\right\}
$$

The operator $\boldsymbol{Z}(\boldsymbol{k})$ is defined in (3.19); it is intimately connected to the spatial Fourier transform of the orbital magnetization. The quantily $B$ in (11.5) is a rather complicated function of polarization and photon wave vectors. Working directly from (11.4),

$$
\mathbf{B}=\frac{1}{2}\left(1+\mathrm{q}^{\prime} / \mathrm{q}\right)\left\{\boldsymbol{\varepsilon}^{\prime} \times \boldsymbol{\varepsilon}-\left(\hat{\mathbf{q}}^{\prime} \times \boldsymbol{\varepsilon}^{\prime}\right) \times(\hat{\mathbf{q}} \times \varepsilon)\right\}+\left(\mathrm{q}^{\prime} / \mathrm{q}\right)\left(\hat{\mathbf{q}}^{\prime}, \boldsymbol{\varepsilon}\right)\left(\hat{\mathbf{q}}^{\prime} \times \varepsilon^{\prime}\right)-\left(\hat{\mathbf{q}} \cdot \boldsymbol{\varepsilon}^{\prime}\right)(\hat{\mathbf{q}} \times \boldsymbol{\varepsilon}) .
$$

Taking the limit $q^{\prime} \rightarrow q$, appropriate for elastic scattering (Bragg diffraction), our result for $\boldsymbol{B}$ is identical apart from an overall sign diflerence with the quantity derived by deBergevin and Brunel (1981). The first term in (11.6) is explicit in (11.4), whereas the three remaining terms arise from the usc of momentum and spin commulation relations in the components in (11.4) that are quadratic in J, c.f. \$10.

The result (11.5) is the basis of our discussions of photon scattering by magnetic materials. Evaluated for $q=q^{\prime}$ the amplitude (11.5) permits a discussion of Bragg diffraction, in which we recover results due largely to deBergevin and Brunel. The inelastic scattering process of main interest is Compton scaltering. For all these experiments there are benefits to be gained in utilizing the polarization of primary and secondary photons. A compact presentation of polarization phenomena, employing a density matrix to handle states of partial polarization, is given in $\$ 3$.

The result (11.5) for $\mathbf{G}$ determines the four components $\alpha$ and $\beta$, listed in $\S 3.3$, from which we obtain the full polarization dependence of the cross-section and the state of polarization of the secondary photon beam by using (3.16) and (3.17). An outline of the steps leading to our expressions for $\alpha$ and $\beta$ begins with the definition (see 3.13)

$$
\mathrm{G}=\rho \beta+\alpha, \sigma
$$


in terms of the unit and Pauli matrices. The next move is to express the polarization dependence that occurs in (11.5) in terms of $2 \times 2$ matrices using the basis defined by Figs (4) and (5). Of the three combinations of $\varepsilon$ and $\varepsilon^{\prime}$ appearing in $\mathbf{G}$ the most complicated is $\mathbf{B}$ defined in (11.6), for which we find,

$$
\mathbf{B}=\left(\begin{array}{ll}
a\left(\hat{\mathbf{q}} \times \hat{\mathbf{q}}^{\prime}\right) & (\mathrm{a}-\mathrm{b}) \hat{\mathbf{q}}+(b \cos \theta-a) \hat{\mathbf{q}}^{\prime} \\
(\mathrm{a}-\cos \theta) \hat{\mathbf{q}}+(1-a) \hat{\mathbf{q}}^{\prime} & (b+1-a)\left(\hat{\mathbf{q}} \times \hat{\mathbf{q}}^{\prime}\right)
\end{array}\right)
$$

with $\mathrm{b}=\left(\mathrm{q}^{\prime} / \mathrm{q}\right)$ and $\mathrm{a}=(1+\mathrm{b}) / 2$. After deriving analogous expressions for $\boldsymbol{\varepsilon}^{\prime} \cdot \boldsymbol{\varepsilon}$ and $\boldsymbol{\varepsilon}^{\prime} \times \boldsymbol{\varepsilon}$, inserting them in (11.5), and using the definition of $\mathbf{G}$ in terms $\alpha$ and $\beta$ the four components listed in $\$ 3.3$ are simply read off.

If the matrix elements of $n(k), S(k)$ and $Z(k)$ that appear in the cross-section are real then the latter, being related to the absolute square of the matrix element of $G$, is quadratic in $\tau$. Hence, the magnetic contribution to the cross-section, weighted by $\tau^{2}$, is small compared with the charge contribution. Another factor reduces even further the ratio of magnetic to charge contributions. Charge scattering engages all electrons whereas magnetic scattering arises from the fraction which are unpaired. If, on the other hand, the charge matrix element, say, is complex (anomalous scallering) the cross-section contains an interference between charge and magnelic contributions, i.e. a term weighted by $\tau$. Such a term is likely to dominate a term weighted by $\tau^{2}$. Another means by which to create an interference between charge and magnetic amplitudes is to employ primary photons with (complex) elliptic polarization. Terms of order $\tau^{2}$ are most likely to be measured at purely magnetic reflections that occur in non-ferromagnetic materials. 


\section{Diffraction}

\subsection{Survey}

In order to set the scene it is useful to note some simple properties of the cross-section (3.16) constructed from the four components of $\alpha$ and $\beta$ listed in (3.22). Looking at the magnetic contributions in these four components, which are identified by the parameter $\tau=\left(\mathrm{E} / \mathrm{mc}^{2}\right)$, it is seen that $\alpha_{1}$ and $\alpha_{2}$ are purely magnetic while $\alpha_{3}$ and $\beta$ are linear combinations of charge and magnetic operators. It is significant that, in $\alpha_{3}$ and $\beta$ the magnetic contributions differ from the charge contributions by a phase factor $i=\sqrt{ }-1$.

Assume in the first instance that the matrix elements of the atomic quantities in $\alpha$ and $\beta$, namely $\mathrm{n}(\mathbf{k}), \mathbf{S}(\mathbf{k})$ and $\mathbf{Z}(\mathbf{k})$, are purely real, and the primary beam is unpolarized, $\mathbf{P}=0$. The cross-section for diffraction is proportional to $\left\{|\langle\alpha\rangle|^{2}+|\langle\beta\rangle|^{2}\right\}$. When the matrix elements in $\langle\alpha\rangle$ and $\langle\beta\rangle$ are real it follows that the magnetic contribution to the crosssection is proportional to $\tau^{2}$, i.e. there is no term proportional to $\tau$. Since $\tau<<1$ (otherwise use of the amplitude (11.5) is not valid) the magnetic content of a mixed (charge and magnetic) Bragg reflection is dominated by the charge contribution. However, pure magnetic reflections can occur in magnetic materials for which the chemical and magnetic structures are different, i.e. materials other than simple ferromagnets. It has been demonstrated with such materials that purely magnetic scaltering is measurable. If the condition on the matrix elements in $\langle\alpha\rangle$ and $\langle\beta\rangle$ is relaxed and they possess imaginary charge components, which is possible in non-centrosymmetric materials or from anomalous scattering, then the diffraction cross-section may contain terms proportional to $\tau$ that are probably larger than terms proportional to $\tau^{2}$.

Polarization of the primary beam has a significant effect on the cross-section. With regard to the influence of linear polarization, note that the matrix $\mathbf{B}$, given in (11.7), which represents the polarization dependence of spin scattering, contains off-diagonal elements. In consequence, $(\sigma-\pi)$ and $(\pi-\sigma)$ events occur through scattering by the spin magnetization. There are also finite off-diagonal elements in $\left(\varepsilon^{\prime} \times \varepsilon\right)$ which represents the polarization dependence of orbital scattering. By contrast, $\left(\varepsilon^{\prime} \cdot \varepsilon\right)$ which represents the polarization dependence of charge scattering is diagonal. From these observations it follows that $(\sigma-\pi)$ or $(\pi-\sigma)$ events reveal purely magnetic scattering.

To appreciate the role of circular polarization in photon scattering by magnetic materials, recollect that circular polarization can be represented by complex polarization vectors. If the matrix elements in $\langle\alpha\rangle$ and $\langle\beta\rangle$ are purely real, the phase difference between 
their charge and magnetic components can be negated by the imaginary part of the complex polarization vectors leading to purely real interference terms. So, it is expected that circular polarization induces interference between charge and magnetic amplitudes, and hence terms of order $\tau$ in the cross-section. Furthermore, changing from right to left-hand polarization, or vice versa, changes the sign of these interference terms leading to the appealing possibility of a difference measurement to isolate the relatively small linear magnetization contribution. This scheme has value only for materials that have mixed (charge and magnetic) reflections, as in a ferromagnet.

Experiments that confirm many of these properties of photon scattering by magnetic solids have been performed by deBergevin and Brunel; a review of their findings and theoretical interpretations is given in deBergevin and Brunel (1986). While these researchers observed magnetic diffraction of order $\tau^{2}$ from bulk antiferromagnetic $\mathrm{NiO}$ using an $\mathrm{x}$-ray tube operated at $1 \mathrm{~kW}$, photon diffraction to investigate the magnetic properties of solids became a realistic experimental method only with the advent of high intensity photon sources, mainly synchrotrons. Photon beams from synchrotron sources are predominantly linearly polarized within the plane of the electron orbit, and elliptically polarized above and below the plane. The resolution in reciprocal space can be superior to that currently available in neutron diffraction.

An early example of new science from photon diffraction studies is the observation by Gibbs et al. (1985) of the effect of the crystal field perturbation on the orientation of magnetic moments in metallic holmium. Fig (7) shows a diffraction pattern of a magnetic satellite in holmium obtained with a polarization analyser that allows isolation of $(\sigma-\pi)$ events which are purely magnetic, as remarked earlier. Use of the filter demonstrates that one peak arises from charge scattering, while the second peak is overwhelmingly magnetic scattering. A number of rare earth metals have now been shown to possess weak structural modulations which accompany the magnetic ordering (Bohr et al. 1989, Bohr et al. 1990). Such diffraction studies demonstrate a high resolution in reciprocal lattice space $\sim 0.001 \AA^{-1}$, using a synchrotron source, which is generally superior to that obtainable in magnetic neutron diffraction. Another example of materials science using photon diffraction is a study of rare earth multilayers (Vettier et al. 1986). In this instance a term linear in the magnetic amplitude was generated by an imaginary component in the charge amplitude, achieved by tuning the wavelength so that an element in the sample gives a high anomalous scattering (resonant scattering).

Magnetic diffraction from ferromagnetic iron of circularly polarized photons has been reported by Collins et al. (1992). They used, for the first time, a white beam and single 
crystal sample, and reversed the direction of the magnetization by an applied field to affect isolation of the polarization induced charge-magnetic interference contribution. The experimental technique offers several attractive features, including a fixed scattering geometry, while several harmonics of a given reflection can be resolved with an energy dispersive detector and measured simultaneously.

The possibility in photon diffraction to separately measure spin and orbital contributions to the amplitude has been realized by Gibbs et al. (1991) in a study of holmium metal and some of their data is displayed in Fig (8). In their work, the ratio of the orbital and spin atomic form factors was deduced from the lincar polarization of the secondary photons. In principle, as we will see, a scattering geometry can be chosen at which the magnetic scattering is either purely orbital or spin in nature. With regard to magnetic neutron diffraction, the observed intensity is always a mixlure of spin and orbital contributions that are not separately obtainable through a choice of scattering geometry (Lander and Stirling, 1992).

\subsection{Pure Charge Scattering}

From the discussion provided in $\$ 5$ it follows that strictly elastic scattering (diffraction) is described by (3.16) and (3.17) when products of operators are taken as products of thermal averages of the operators. So, for example, the term $\beta^{+}(\alpha . P)$ in $(3.16)$ is $\left\langle\beta^{+}\right\rangle\langle\alpha>$. P for strictly elastic scattering achieved in Bragg diffraction. The same interpretation holds for terms in (3.17) for the polarization of the secondary beam.

As a first example of the use of (3.16) and (3.17) consider diffraction from a nonmagnetic material. Referring to (3.22), we have for this case

$$
\alpha_{1}=\alpha_{2}=0
$$

and

$$
\begin{aligned}
& \alpha_{3}=\frac{1}{2}(1-\cos \theta) n(\mathbf{k}) \\
& \beta=\frac{1}{2}(1+\cos \theta) n(\mathbf{k}) .
\end{aligned}
$$

Let,

$$
\langle\mathrm{n}(\mathbf{k})\rangle=\mathrm{N}(\mathbf{k}) \text {, }
$$


which means that $N(k)$ is the average particle density for the target assembly, and it is usually a complex quantity, $N=N^{\prime}+i N^{\prime \prime}$.

For the cross-section (3.16) we find the value,

$$
\frac{\mathrm{d} \sigma}{\mathrm{d} \Omega}=\frac{1}{2} \mathbf{r}_{\mathrm{e}}^{2}|\mathrm{~N}(\mathbf{k})|^{2}\left\{1+\cos ^{2} \theta+\mathrm{P}_{3} \sin ^{2} \theta\right\}
$$

It is interesting to observe that (a) the cross-section is independent of circular polarization and of the Stokes parameter $P_{1}$, and (b) the cross-section for photons polarized perpendicular to the scattering plane ( $\sigma$ polarizalion, $\mathrm{P}_{3}=+1$ ), defined by $\boldsymbol{q}$ and $\boldsymbol{q}^{\prime}$, is larger than for photons in the scattering plane ( $\pi$ polarization, $\left.\mathrm{P}_{3}=-1\right)$.

Stokes parameters for the secondary beam follow from (3.17) and (12.2). For the special case $P_{1}=P_{2}=0, P_{3} \neq 0$, we readily obtain

$$
\mathrm{P}_{1}^{\prime}=\mathrm{P}_{2}^{\prime}=0
$$

while $\mathrm{P}_{3}$ is independent of $\mathrm{P}_{1}$ and $\mathrm{P}_{2}$ and has the value,

$$
P_{3}=\left(\frac{\sin ^{2} \theta+P_{3}\left(1+\cos ^{2} \theta\right)}{1+\cos ^{2} \theta+P_{3} \sin ^{2} \theta}\right)
$$

When $P_{3}=0, P_{3}^{\prime}>0$ so there is a finite linear polarization along the $\xi$-axis in Fig. (4). The values $P_{3}^{\prime}= \pm 1$ are obtained for $P_{3}= \pm 1$ for any value of $\theta$. For $\theta=\pi / 2, P_{3}^{\prime}=1$ for any value of $\mathrm{P}_{3}$, i.e. $90^{\circ}$ charge scattering produces complete $\sigma$-polarization of the secondary beam. This and the other findings for linear polarization are in accord with the discussion of polarization effects in Thomson scaltering provided in $\$ 3.1$. It can be shown that circular polarization in the secondary beam occurs only if the primary beam contains circular polarization, i.e. $P_{2}^{\prime} \neq 0$ only if $P_{2} \neq($ ).

\subsection{Unpolarized Primary Beam}

As the second case we choose to examine diffraction of an unpolarized photon beam by a magnetic crystal. When $q=q^{\prime}$ the operator $Z(k)$ reduces to, 


$$
\hbar \mathbf{Z}(\mathbf{k})=\frac{i}{q^{2}} \sum_{j} \exp \left(i \mathbf{k} \cdot \mathbf{R}_{\mathbf{j}}\right)\left(\mathbf{k} \times \mathbf{p}_{\mathbf{j}}\right),
$$

and for elastic events,

$$
Z(\mathbf{k})=-\frac{k^{2}}{q^{2}} \mathbf{T}(\mathbf{k}),
$$

in which

$$
k^{2}=2 q^{2}(1-\cos \theta),
$$

and $T(k)$ is related to the orbital magnetization through eqn (3.20). Eqn (12.4) shows that $k . Z=0$. The quantities $\alpha_{1}, \alpha_{2}, \alpha_{3}$ and $\beta$ are obtained from (3.22) by taking $\mathrm{a}=\mathrm{b}=1$;

$$
\begin{aligned}
& \alpha_{1}=\frac{-i}{2} \tau(1-\cos \theta)\left(\hat{\mathbf{q}}-\hat{\mathbf{q}}^{\prime}\right) \cdot \mathbf{S}(\mathbf{k}) \\
& \alpha_{2}=\frac{1}{2} \tau\left(\hat{\mathbf{q}}+\hat{\mathbf{q}}^{\prime}\right) \cdot(\mathbf{Z}(\mathbf{k})-(1-\cos \theta) \mathbf{S}(\mathbf{k})) \\
& \alpha_{3}=\frac{1}{2}(1-\cos \theta) \mathrm{n}(\mathbf{k})-\frac{i}{2} \tau\left(\hat{\mathbf{q}} \times \hat{\mathbf{q}}^{\prime}\right) \cdot \mathbf{Z}(\mathbf{k})
\end{aligned}
$$

and

$$
\beta=\frac{1}{2}(1+\cos \theta) n(\mathbf{k})+\frac{i}{2} \tau\left(\hat{\mathbf{q}} \times \hat{\mathbf{q}}^{\prime}\right) \cdot(\mathbf{Z}(\mathbf{k})-2 \mathbf{S}(\mathbf{k})) .
$$

From (3.20),

$$
\langle\mathbf{T}(\mathbf{k})\rangle=-\frac{1}{2 \mu_{\mathrm{B}}} \int \mathrm{d} \mathbf{r} \exp (\mathrm{ik} \cdot \mathbf{r})\left\{\hat{\mathbf{k}} \times\left(\left\langle\mathbf{M}_{\ell}(\mathbf{r})\right\rangle \times \hat{\mathbf{k}}\right)\right\}
$$

and we choose to write

$$
\langle\mathbf{S}(\mathbf{k})\rangle=-\frac{1}{2 \mu_{\mathrm{B}}} \int \mathrm{d} \mathbf{r} \exp (\mathrm{ik} . \mathbf{r})\left\langle\mathbf{M}_{\mathrm{s}}(\mathbf{r})\right\rangle .
$$

For a perfect magnetic crystal, $\left\langle M_{\ell}(r)\right\rangle$ and $\left\langle M_{s}(r)\right\rangle$ are periodic functions that can be expressed as Fourier series in reciprocal space.

The position of a unit cell in the crystal is defined by a lattice vector $\ell$ and the cell volume is denoted by $\mathrm{v}_{o}$. An atom position $\boldsymbol{R}_{j}=\ell+d$, where $d$ defines the position within a 
unit cell. The site $d=0$ coincides with the corner of the cell, so there are $(r-1)$ non-null vectors $\boldsymbol{d}$. Vectors of the reciprocal lattice $\{\tau\}$ satisfy

$$
\exp (i l . \tau)=1 ; \text { for all } \ell \text {. }
$$

Because $\left\langle M_{\ell}(r)\right\rangle$ is periodic we can write,

$$
-\frac{1}{2 \mu_{\mathrm{B}}} \int_{\Omega} \mathrm{d} \mathbf{r} \exp (\mathrm{ik}, \mathbf{r})\left\langle\mathbf{M}_{\ell}(\mathbf{r})\right\rangle=\frac{(2 \pi)^{3}}{\mathrm{v}_{\mathrm{o}}} \sum_{\tau} \delta(\mathbf{k}-\tau) \mathbf{F}_{\ell}(\tau),
$$

and a similar expression for the spin density. We refer to $F_{\ell}(\tau)$ and $F_{s}(\tau)$ as magnetic unitcell structure factors for orbital and spin magnetizations, respectively. Inverting (12.10) we get,

$$
\left(-1 / 2 \mu_{\mathrm{B}}\right)\left\langle\mathbf{M}_{\ell}(\mathbf{r})\right\rangle=\frac{1}{v_{\mathrm{o}}} \sum_{\tau} \exp (-\mathrm{i} \tau \cdot \mathbf{r}) \mathbf{F}_{\ell}(\tau),
$$

and the conjugate relation,

$$
\mathbf{F}_{\ell}(\tau)=\int_{\text {cell }} \mathrm{d} \mathbf{r} \exp (\mathrm{i} \tau \cdot \mathbf{r})\left\langle\mathbf{M}_{\ell}(\mathbf{r})\right\rangle /\left(-2 \mu_{\mathrm{B}}\right),
$$

together with analogous relations for the spin magnetization.

Consider a simple magnet with one atom per unit cell. At least for moderate values of $k$, the atomic structure factors for spin and orbital contributions to the total moment are almost the same. Let $F(k)$ be the atomic form factor, with the standard normalization $F(0)=1$. So, to a good approximation,

$$
\mathbf{F}_{\ell}(\mathbf{k})=\frac{1}{2}\langle\mathbf{L}\rangle \mathrm{F}(\mathrm{k})
$$

and

$$
\mathbf{F}_{\mathrm{s}}(\mathbf{k})=\langle\mathbf{S}\rangle \mathrm{F}(\mathrm{k})
$$

where $L$ and $S$ are the (total) orbital and spin angular momentum operators for the magnetic ion. For a 3d-transition metal ion it is usual to define a gyromagnetic factor $g_{o}$ by,

$$
\langle\mathbf{L}\rangle=\left(\mathrm{g}_{\mathrm{o}}-2\right)\langle\mathbf{S}\rangle \text {, }
$$


in which case the ratio of $F_{\ell}$ to $F_{s}$ is simply $\left(g_{o}-2\right) / 2$. On the other hand, a rare earth ion is characterized by the total angular momentum $J=L+S$. For a given $J$ - manifold, $L=g_{\ell} J$ and $S=g_{s} J$ with

$$
\mathrm{g}_{\ell}=(2-\mathrm{g}), \mathrm{g}_{\mathrm{s}}=(\mathrm{g}-1)
$$

in which $g$ is the Lande factor.

Finally we note that all the cross-sections involve products of densities of the form,

$$
\left(-1 / 2 \mu_{\mathrm{B}}\right)\{\mathrm{N}(\mathbf{k})\}^{*} \int \mathrm{d} \mathbf{r} \exp (\mathrm{ik}, \mathbf{r})\left\langle\mathbf{M}_{\ell}(\mathbf{r})\right\rangle=\mathrm{N}_{\mathrm{w}} \frac{(2 \pi)^{3}}{\mathrm{v}_{\mathrm{o}}} \sum_{\tau} \delta(\mathbf{k}-\boldsymbol{\tau}) \mathrm{F}_{\mathrm{c}}^{*}(\tau) \mathbf{F}_{\ell}(\boldsymbol{\tau})
$$

where $N_{o}$ is the number of unit cells and $F_{c}(k)$ is the charge unit cell structure factor. If the chemical and magnetic structures are different there exist $\tau$ for which $F_{\ell}(\tau)=0$ and/or $\boldsymbol{F}_{s}(\tau)=0$ while $\mathbf{F}_{\mathrm{c}}(\tau) \neq 0$, and vice versa, i.e. $\{\tau\}$ generally comprises pure charge and pure magnetic reflections, and mixed reflections.

In subsequent expressions for diffraction cross-sections the factor

$$
\mathrm{N}_{\mathrm{o}} \frac{(2 \pi)^{3}}{\mathrm{v}_{\mathrm{o}}} \sum_{\tau} \delta(\mathrm{k}-\tau)
$$

on the right-hand side of $(12,14)$ is omitted, simply to minimize the notation. The factor cancels in expressions for the Stokes vector for the secondary beam. Thus, subsequent expressions for cross-sections and $\mathbf{P}^{\prime}$ are understood to refer to a Bragg reflection defined by $\{\tau\}$. With our chosen notation, $\langle\alpha\rangle$ and $\langle\beta\rangle$ are linear combinations of the unit cell structure factors for charge $\left(F_{c}\right)$, spin $\left(F_{s}\right)$ and orbital $\left(\mathbf{F}_{f}\right)$ densities. Working directly from (12.6),

$$
\begin{aligned}
& <\alpha_{1}>=-\frac{i}{2} \tau(1-\cos \theta)\left(\hat{\mathbf{q}}-\hat{\mathbf{q}}^{\prime}\right) \cdot \mathbf{F}_{\mathrm{s}} \\
& <\alpha_{2}>=-\tau(1-\cos \theta)\left(\hat{\mathbf{q}}+\hat{\mathbf{q}}^{\prime}\right) \cdot\left(\mathbf{F}_{\mathrm{l}}+\frac{1}{2} \mathbf{F}_{\mathrm{s}}\right) \\
& <\alpha_{3}>=(1-\cos \theta)\left\{\frac{1}{2} \mathrm{~F}_{\mathrm{c}}+\mathrm{i} \tau\left(\hat{\mathbf{q}} \times \hat{\mathbf{q}}^{\prime}\right) \cdot \mathbf{F}_{\mathrm{l}}\right\} \cdot \mathrm{s}
\end{aligned}
$$

and

$$
<\beta>=\frac{1}{2}(1+\cos \theta) \mathrm{F}_{c}-\mathrm{i} \tau\left(\hat{\mathbf{q}} \times \hat{\mathbf{q}}^{\prime}\right) \cdot\left\{(1-\cos \theta) \mathbf{F}_{l}+\mathbf{F}_{s}\right\}
$$


In general, $F_{c}, F_{l}$ and $\mathbf{F}_{\mathrm{s}}$ are complex quantities. For a centrosymmetric crystal structure, $F_{t}$ and $\mathbf{F}_{\mathrm{s}}$ are purely real, as is $\mathrm{F}_{\mathrm{c}}$ unless the photon energy is tuned to a resonance for an atom in the unit cell. An example of a structure for which $\mathbf{F}_{t}$ and $\mathbf{F}_{s}$ can be complex is the hexagonal close-packed structure adopted by rare-earth metals. It is interesting to observe that if the spin and orbital moments are perpendicular to the plane of scattering defined by $\mathbf{q}$ and $\mathbf{q}^{\prime}$ then $\left\langle\alpha_{1}\right\rangle=\left\langle\alpha_{2}\right\rangle=0$, while if $\mathbf{F}_{\mathrm{S}}$ and $\mathbf{F}_{l}$ lie in the plane $\left\langle\alpha_{3}\right\rangle$ and $\langle\beta\rangle$ contain only the charge density. Approaching the forward scattering position, $\theta \rightarrow 0$, all the components of $\langle\alpha\rangle$ tend to zero, and $\langle\beta\rangle$ contains only the charge density. With the component of $\mathbf{F}_{\mathrm{s}}$ in the plane of scattering arranged perpendicular to $\mathbf{k}$ it follows that $\left\langle\alpha_{1}\right\rangle=0$, whereas when the components of $\mathbf{F}_{\mathrm{s}}$ and $\mathbf{F}_{1}$ in the plane are arranged parallel to $\mathbf{k}$ one has $\left\langle\alpha_{2}\right\rangle=0$.

With an unpolarized primary beam the diffraction cross-section for the scattering geometry depicted in Fig (9) is

$$
\begin{aligned}
& \left(\frac{d \sigma}{d \Omega}\right)_{0}=\mathbf{r}_{\mathrm{e}}^{2}\left\{\operatorname { s i n } ^ { 4 } ( \theta / 2 ) \left[\tau^{2}\left|\left(\hat{\mathrm{q}}-\hat{\mathrm{q}}^{\prime}\right) \cdot \mathbf{F}_{\mathrm{s}}\right|^{2}+\tau^{2}\left|\left(\hat{\mathbf{q}}+\hat{\mathbf{q}}^{\prime}\right) \cdot\left(2 \mathrm{~F}_{l}+\mathbf{F}_{\mathrm{s}}\right)\right|^{2}\right.\right. \\
& \left.\left.+\left|\mathrm{F}_{\mathrm{c}}+2 \mathrm{i} \tau \sin \theta \mathrm{F}_{l}^{\mathrm{y}}\right|^{2}\right]+\frac{1}{4}\left|(1+\cos \theta) \mathrm{F}_{\mathrm{c}}-2 \mathrm{i} \tau \sin \theta\left((1-\cos \theta) \mathrm{F}_{l}^{\mathrm{y}}+\mathrm{F}_{\mathrm{s}}^{\mathrm{y}}\right)\right|^{2}\right\} .
\end{aligned}
$$

In this expression, the superscript y denotes a component perpendicular to the plane of scattering in the direction opposite to the $\xi$ - axis. An interference between charge and magnetic amplitudes is possible at mixed Bragg reflections for which there are components of $\mathbf{F}_{\mathrm{s}}$ and $\mathbf{F}_{l}$ perpendicular to the plane. For a pure magnetic reflection, such as a magnetic satellite for a rare earth metal, $F_{c}=0$ and in general both $\mathbf{F}_{\mathrm{s}}$ and $\mathbf{F}_{l}$ are different from zero. Above the ordering temperature $\mathbf{F}_{t}=\mathbf{F}_{\mathrm{S}}=0$, and the cross-section is consistent with the classical expression (3.2) for coherent scattering by a charge distribution.

It is interesting to examine the linear polarization of a purely magnetic diffraction signal. In (12.15) $\mathrm{F}_{\mathrm{c}}=0$, and for $\mathrm{P}_{3}^{\prime}$ (3.17) yields the value,

$$
\begin{aligned}
\left(\frac{d \sigma}{d \Omega}\right) P_{3}^{\prime} & =-\tau^{2}(1-\cos \theta)^{2} \operatorname{Re} \cdot\left\{\left(2 \cos \frac{\theta}{2}\right)^{2} F_{l}^{y}\left(F_{s}^{y}+(1-\cos \theta) F_{l}^{y}\right)^{*}\right. \\
& \left.+\left(\hat{\mathbf{q}}-\hat{\mathbf{q}}^{\prime}\right) \cdot \mathbf{F}_{\mathrm{s}} *\left(\hat{\mathbf{q}}+\hat{\mathbf{q}}^{\prime}\right) \cdot\left(\mathbf{F}_{l}+\frac{1}{2} \mathbf{F}_{\mathrm{s}}\right)\right\}
\end{aligned}
$$


where the cross-section on the left-hand side is given by $(12,16)$ with $F_{c}=0$. Observe that when the moment is perpendicular to the plane; $\mathrm{P}_{3}^{\prime}$ is proportional to the orbital magnetization, and for the extreme case in which there is no spin magnetization $\mathrm{P}_{3}^{\prime}=-1$ for all qualifying reflections. Conversely, with $F_{c}=F_{t}=0$ and $F_{s}$ perpendicular to the plane $\mathrm{P}_{3}^{\prime}=0$. The last finding is a special case of the result,

$$
P_{3}^{\prime}=\frac{-\sin \theta \sin 2 \varphi}{\left\{1-\cos \theta \cos 2 \varphi+2\left(\cot \beta \cot \frac{1}{2} \theta\right)^{2}\right\}}
$$

in which $\mathbf{F}_{\mathrm{s}}$ makes an angle $\beta$ with the $y$-axis, perpendicular to the plane of scattering, and the projection of $\mathbf{F}_{\mathrm{s}}$ in the plane makes an angle $\varphi$ with the scattering vector $\mathbf{k}$; see Fig. (9). Note that $\mathrm{P}_{3}=0$ when the projection of $\mathbf{F}_{\mathrm{s}}$ in the plane is parallel or perpendicular to $\mathbf{k}$, as is evident from the gencral expression (12.17) with $\mathbf{F}_{t}=0$. These various results for $\mathrm{P}_{3}$ at a purely magnetic reflection serve to illustrate the value in analysing the linear polarization (Gibbs et al. 1988, 1991) and the striking dilferences in photon scattering between the spin and orbital magnetization components.

Turning now to the cross-section for mixed reflections, the appropriate expression evaluated to leading-order in $\tau$ is

$$
\begin{aligned}
\frac{d \sigma}{d \Omega}=r_{c}^{2} & \left\{\frac{1}{2}\left(1+\cos ^{2} \theta\right)\left|F_{c}\right|^{2}+\tau \sin \theta(1+\cos \theta) \operatorname{Im} \cdot\left(F_{c}^{*} F_{s}^{y}\right)\right. \\
& \left.+\tau \sin 2 \theta(1-\cos \theta) \operatorname{lm} .\left(F_{c}^{*} F_{l}^{y}\right)\right\} .
\end{aligned}
$$

The interference contribution is seen to involve just the components of $\mathbf{F}_{l}$ and $\mathbf{F}_{\mathrm{s}}$ that are perpendicular to the plane of scallering. At least with regard to experiments performed at synchrotron sources, where the photons are predominantly polarized in the plane of the orbit, (12.19) is actually misleading. The complete expression (12.21) shows that, the orbital term in the cross-section vanishes for $\mathrm{P}_{3}=1$ and, 10 a good approximation, this is realized at a synchrotron source when the plane of scattering is perpendicular to the plane containing the electron beam orbil.

The calculation of $\mathbf{P}^{\prime}$ reveals that $\mathrm{P}_{1}^{\prime}$ and $\mathrm{P}_{2}^{\prime}$ arc proportional to the magnetic amplitude, and hence they are small quantitics. On the other hand, $\mathrm{P}_{3}$ contains both pure charge and mixed amplitudes and the pure charge contribution is, of course, consistent with (12.3). 


\subsection{Linear Polarization}

In this section the focus is on features due to linear polarization in the primary photon beam, and $\mathbf{P}=\left(0,0, \mathrm{P}_{3}\right)$. The cross-section of interest is,

$$
\frac{\mathrm{d} \sigma}{\mathrm{d} \Omega}=\left(\frac{\mathrm{d} \sigma}{\mathrm{d} \Omega}\right)_{0}+2 \mathrm{r}^{2} \mathrm{P}_{3}\left\{\operatorname{Re} .\left(<\beta>*<\alpha_{3}>\right)-\operatorname{Im} .\left(<\alpha_{1}>*<\alpha_{2}>\right)\right\}
$$

where $(\mathrm{d} \sigma / \mathrm{d} \Omega)_{0}$ is the cross-section for unpolarized photons given in (12.16), and the components of $\langle\alpha\rangle$ and $\langle\beta\rangle$ arc provided in (12.15). Perhaps the first thing to observe is that, $\left.(<\beta\rangle^{*}<\alpha_{3}>\right)$ involves only those components of the magnetization that are perpendicular to the plane of scattering (the $y$-axis in Fig (9)) while $\left(<\alpha_{1}>^{*}<\alpha_{2}>\right.$ ) involves only those components of the magnetization that lic in the planc. Furthermore, $\left.\left.\left(<\alpha_{1}\right\rangle^{*}<\alpha_{2}\right\rangle\right)$ vanishes if the components of $\mathbf{F}_{\mathrm{s}}$ and $\mathbf{F}_{i}$ in the plane are either parallel or perpendicular to the scattering vector $\mathbf{k}$.

The product $\left.\left(\left\langle\alpha_{1}\right\rangle^{*}<\alpha_{2}\right\rangle\right)$ is proportional to $\tau^{2}$, and consequently it does not contribute to the leading-order interference between charge and magnetic amplitudes. The results (12.19) and (12.20) produce the expression, correct to order $\tau$,

$$
\begin{aligned}
\frac{\mathrm{d} \sigma}{\mathrm{d} \Omega}=\mathrm{r}_{\mathrm{c}}^{2} & \left\{\left(1+\frac{1}{2}\left(\mathrm{P}_{3}-1\right) \sin ^{2} \theta\right)\left|\mathrm{F}_{\mathrm{c}}\right|^{2}+\tau\left(1-\mathrm{P}_{3}\right) \sin 2 \theta(1-\cos \theta) \operatorname{Im} \cdot\left(\mathrm{F}_{\mathrm{c}}^{*} \mathrm{~F}_{\mathrm{l}}^{\mathrm{y}}\right)\right. \\
& \left.+\tau \sin \theta\left(\left(1+\mathrm{P}_{3}\right)+\cos \theta\left(1-\mathrm{P}_{3}\right)\right) \operatorname{Im} \cdot\left(\mathrm{F}_{\mathrm{c}}^{*} \mathrm{~F}_{\mathrm{s}}^{\mathrm{y}}\right)\right\}
\end{aligned}
$$

Observe that, the pure charge contribution is consistent with (7.10) for the classical limit of scattering by a free electron. The orbital contribution to (12.21) vanishes for $\sigma$-polarization when the linear polarization of the primary beam is perpendicular to the plane of scattering, and oppositely aligned to the $y$-axis, c.f, Figs $(4,9)$. In consequence, linear polarization permits a separate determination of the spin and orbital contributions to the cross-section.

Some general fealures of the linear polarization of the secondary beam, described by the Stokes parameter $\mathrm{P}_{3}^{\prime}$, can be deduced from the structure of the polarization matrices that occur in the magnetic scattering amplitude. The spin component contains B, displayed in (11.7), and the orbital component contains,

$$
\varepsilon^{\prime} \times \varepsilon=\left(\begin{array}{cc}
0 & \hat{\mathbf{q}} \\
-\hat{\mathbf{q}}^{\prime} & \hat{\mathbf{q}}^{\prime} \times \hat{\mathbf{q}}
\end{array}\right) .
$$


Evidently, if $\mathbf{F}_{\mathrm{S}}$ and $\mathbf{F}_{l}$ are perpendicular to the plane of scattering $(\sigma-\pi)$ and $(\pi-\sigma)$ magnetic events are forbidden, and $\mathrm{P}_{3}^{\prime}= \pm 1$ for $\mathrm{P}_{3}= \pm 1$. On the other hand, if $\mathbf{F}_{\mathrm{s}}$ and $\mathbf{F}_{\mathrm{l}}$ lie in the plane $(\sigma-\sigma)$ and $(\pi-\pi)$ events are forbidden, and $\mathrm{P}_{3}^{\prime}= \pm 1$ for $\mathrm{P}_{3}=\mp 1$.

The linear polarization of the sccondary beam is determined by the expression,

$$
\begin{aligned}
\left(\frac{d \sigma}{d \Omega}\right) P_{3}^{\prime} & =P_{3}\left(\frac{d \sigma}{d \Omega}\right)_{0}+2 r_{\mathrm{e}}^{2}\left\{\operatorname{Re} \cdot\left(<\beta>*<\alpha_{3}>\right)+\operatorname{Im} \cdot\left(<\alpha_{1}>^{*}<\alpha_{2}>\right)\right. \\
& \left.-P_{3}\left(\left|<\alpha_{1}>\right|^{2}+\left|<\alpha_{2}>\right|^{2}\right)\right\} .
\end{aligned}
$$

Here, $(\mathrm{d} \sigma / \mathrm{d} \Omega)_{0}$ is the cross-section (12.16) and the cross-section on the left-hand side is given by $(12.2())$. In discussing the latter, we have noted the salient features of $\left(\langle\beta\rangle^{*}<\alpha_{3}>\right)$ and $\left.\left(\left\langle\alpha_{1}\right\rangle^{*}<\alpha_{2}\right\rangle\right)$. The only point that needs to be added with regard to (12.22) is that $\left.\left|<\alpha_{1}\right\rangle\right|^{2}$ and $\left.\left|<\alpha_{2}\right\rangle\right|^{2}$ are both of order $\tau^{2}$, and both vanish if the magnetization is perpendicular to the plane of scattering. Evaluated for pure charge scattering, (12.22) agrees with (12.3). For the casc of mixed reflections,

$$
\begin{aligned}
\left(\frac{d \sigma}{d \Omega}\right) P_{3}^{\prime} & =r_{c}^{2}\left\{\frac{1}{2}\left(\sin ^{2} \theta+P_{3}\left(1+\cos ^{2} \theta\right)\right)\left|F_{c}\right|^{2}+\tau\left(P_{3}-1\right) \sin 2 \theta(1-\cos \theta) \operatorname{Im} .\left(F_{c}^{*} F_{l}^{y}\right)\right. \\
& \left.+\tau \sin \theta\left(1+P_{3}+\cos \theta\left(P_{3}-1\right)\right) \mathrm{Im} .\left(F_{c}^{*} F_{s}^{y}\right)\right\},
\end{aligned}
$$

with the result (12.21) for the cross-section on the left-hand side. Note that $(12,21)$ and (12.23) obey the requirement $\mathrm{P}_{3}^{\prime}= \pm 1$ for $\mathrm{P}_{3}= \pm 1$.

Expressions for the cross-section and secondary polarization are algebraically quite complicated even for the special case of pure magnetic rellections, i.e. reflections for which $\mathrm{F}_{\mathrm{c}}(\mathrm{k})=0$ and the magnetic structure factors are finite. In view of this, we elect to consider special limits of the expressions beginning with the magnetization perpendicular to the plane of scattering, defined by $\mathrm{q}$ and $\mathrm{q}^{\prime}$. The cross-section for this case is,

$$
\begin{aligned}
\left(\frac{d \sigma}{d \Omega}\right)= & \left(\tau r_{c} \sin \theta\right)^{2}\left\{\left.2\left(1-P_{3}\right)(1-\cos \theta)|(1-\cos \theta)| F_{l}^{y}\right|^{2}\right. \\
& \left.\left.+\operatorname{Rc} \cdot\left(F_{i}^{y} F_{s}^{y^{*}}\right)\right]+\left|F_{s}^{y}\right|^{2}\right\}
\end{aligned}
$$

and the corresponding result for $\mathrm{P}_{3}^{\prime}$ is 


$$
\begin{aligned}
\left(\frac{d \sigma}{d \Omega}\right) P_{3}^{\prime} & =\left(\tau r_{e} \sin \theta\right)^{2}\left\{2 ( P _ { 3 } - 1 ) ( 1 - \operatorname { c o s } \theta ) \left[(1-\cos \theta)\left|F_{l}^{y}\right|^{2}\right.\right. \\
& \left.\left.+\operatorname{Re} \cdot\left(F_{l}^{y} F_{s}^{y^{*}}\right)\right]+P_{3}\left|F_{s}^{y}\right|^{2}\right\} .
\end{aligned}
$$

Hence, when the magnetization is perpendicular to the plane, and the primary radiation is $\sigma$-polarized, the cross-section measures the spin magnetization. A similar finding holds for charge-magnetic scattering. The pair of results (12.24) and (12.25) obey $\mathrm{P}_{3}^{\prime}= \pm 1$ for $\mathrm{P}_{3}= \pm 1$.

The last case we consider is that of pure magnetic reflections $\left(F_{c}=0\right)$ with the magnetization confined to the plane of scatcring, In this instance, $\left\langle\alpha_{3}\right\rangle=\langle\beta\rangle=0$. These results lead directly to the finding $\mathrm{P}_{3}^{\prime}= \pm 1$ when $\mathrm{P}_{3}=\mp 1$. The foregoing result is manifest in the following pair of relations,

$$
\frac{\mathrm{d} \sigma}{\mathrm{d} \Omega}=\mathrm{r}_{e}^{2}\left\{\left|<\alpha_{1}>\right|^{2}+\left|<\alpha_{2}>\right|^{2}-2 \mathrm{P}_{3} \operatorname{Im} \cdot\left(<\alpha_{1}>*<\alpha_{2}>\right)\right\}
$$

and

$$
\left(\frac{\mathrm{d} \sigma}{\mathrm{d} \Omega}\right) \quad \mathrm{P}_{3}^{\prime}=-\mathrm{r}_{\mathrm{c}}^{2}\left\{\mathrm{P}_{3}\left(\left|<\alpha_{1}>\right|^{2}+\left|<\alpha_{2}>\right|^{2}\right)-2 \operatorname{Im} .\left(<\alpha_{1}>*<\alpha_{2}>\right)\right\} \text {. }
$$

On developing the expression for the cross-section one arrives at the result,

$$
\begin{aligned}
\frac{\mathrm{d} \sigma}{\mathrm{d} \Omega}= & \left(\tau \mathrm{r}_{\mathrm{e}} \sin ^{2} \frac{1}{2} \theta\right)^{2}\left(\left|\left(\hat{\mathbf{q}}-\hat{\mathbf{q}}^{\prime}\right) \cdot \mathbf{F}_{\mathrm{s}}\right|^{2}+\left|\left(\hat{\mathbf{q}}+\hat{\mathbf{q}}^{\prime}\right) \cdot\left(2 \mathbf{F}_{l}+\mathbf{F}_{\mathrm{s}}\right)\right|^{2}\right. \\
& +2 \mathrm{P}_{3} \operatorname{Re} \cdot\left[\left(\hat{\mathbf{q}}-\hat{\mathbf{q}}^{\prime}\right) \cdot \mathbf{F}_{\mathrm{s}}^{*}\left(\hat{\mathbf{q}}+\hat{\mathbf{q}}^{\prime}\right) \cdot\left(2 \mathbf{F}_{l}+\mathbf{F}_{\mathrm{s}}\right)\right\},
\end{aligned}
$$

from which the corresponding result for $\mathrm{P}_{3}^{\prime}$ is quite readily derived. Inspection of these expressions leads to the view that, the geometry for which (12.26) - (12.28) apply is not particularly useful for a comparative study of spin and orbital magnetism in solids.

To gain some more insight to (12.28), and the companion expression for $\mathrm{P}_{3}^{\prime}$, let us use a model appropriate for some rare earth magnets: 


$$
\mathbf{F}_{\mathrm{s}}(\mathbf{k})=\mathrm{g}_{\mathrm{s}} \Delta(\mathbf{k})<\mathrm{J}>
$$

and

$$
\mathbf{F}_{t}(\mathbf{k})=1 / 2 \mathrm{~g}_{t} \Delta(\mathbf{k})<\mathrm{J}>
$$

where $\Delta(\mathbf{k})$ describes the moment configuration and $\mathrm{g}_{\mathrm{s}}$ and $\mathrm{g}_{\mathrm{l}}$ are related to the Lande factor, c.f. §12.3. The corresponding cross-section is,

$$
\begin{aligned}
& \left(\frac{d \sigma}{d \Omega}\right)=2\left(\tau r_{\mathrm{e}}<\mathrm{J}>\sin ^{2} \frac{\theta}{2}\right)^{2}|\Delta(\mathrm{k})|^{2}\left\{\mathrm{~g}_{\mathrm{s}}^{2}(1-\cos (\theta+2 \varphi))\right. \\
& \left.+\mathrm{g}_{s}\left(\mathrm{P}_{3}\left(\mathrm{~g}_{l}+\mathrm{g}_{\mathrm{s}}\right)-\mathrm{g}_{\mathrm{s}}\right) \sin \theta \sin 2 \varphi+\mathrm{g}_{l}\left(\mathrm{~g}_{\mathrm{l}}+2 \mathrm{~g}_{\mathrm{s}}\right)(1+\cos \theta) \sin ^{2} \varphi\right),
\end{aligned}
$$

in which $\varphi$ is the angle between $\langle\mathrm{J}>$, which is in the plane of scatering, and the scattering vector $\mathbf{k}$. It is interesting to observe that the cross-section is independent of the polarization when $\langle\mathrm{J}\rangle$ is parallel or perpendicular to $\mathbf{k}$.

\subsection{Circular Polarization}

The influence on scatlering from a magnetic material of circular polarization in the primary beam is illustrated in the result (7.9) for the cross-section for scattering by an aligned electron. Unlike linear polarization, circular polarization induces interference between the charge and magnelic scaltering amplitudes. In consequence, use of circular polarization is a valuable method for the measurement of magnelic scattering, providing as it does both the sign and magnitude of the amplitude, good signal-to-noise by a difference technique, and potentially betler flexibility than use of anomalous charge seatlering. However, it is restricted to the investigation of mixed charge-magnetic reflections.

The existence in the cross-section of charge-magnetic interference induced by circular polarization is apparent when we look at terms to be added to the cross-section $(12.20)$. From (3.16) it is found that the additional terms are,

$$
2 \mathrm{P}_{2} \mathrm{I}_{\mathrm{c}}^{2}\left\{\mathrm{Re} .\left(<\beta>^{*}<\alpha_{2}>\right)+\operatorname{Im} .\left(<\alpha_{1}>^{*}<\alpha_{3}>\right)\right\} .
$$

Turning to $(12.15),\left\langle\alpha_{1}\right\rangle$ and $\left\langle\alpha_{2}\right\rangle$ are purely magnelic while $\langle\beta\rangle$ and $\left\langle\alpha_{3}\right\rangle$ are combinations of charge and magnetic terms. One general observation is that $\left\langle\alpha_{1}\right\rangle$ and $\left\langle\alpha_{2}\right\rangle$ vanish if $\mathbf{F}_{\mathrm{S}}$ and $\mathbf{F}_{i}$ are perpendicular to the plane of scaltering. Hence, charge-magnetic interference induced by circular polarization does not contribute to the cross-section unless there is a component of the magnelization in the plane, preciscly as in (7.9) for scattering by a polarized electron.

On devcloping the quantitics in (12.30) to leading-order in $\tau$, the contribution to the cross-section induced by circular polarization is found to be, 


$$
-r_{e}^{2} \tau(1-\cos \theta) P_{2} R c \cdot\left\{F_{i}^{*}\left((1+\cos \theta)\left(\hat{\mathbf{q}}+\hat{\mathbf{q}}^{\prime}\right) \cdot \mathbf{F}_{1}+\left(\hat{\mathbf{q}} \cos \theta+\hat{\mathbf{q}}^{\prime}\right) \cdot \mathbf{F}_{\mathrm{s}}\right)\right\} .
$$

The cross-section for a primary beam described by a Stokes vector $\mathbf{P}=\left(0, \mathrm{P}_{2}, \mathrm{P}_{3}\right)$ is the sum of (12.21) and (12.31). It is interesting to observe that the spin contribution in (12.31) is precisely the same as the spin contribution in (7.10) for the classical non-relativistic cross-section for scattering by a single free clectron that is at rest before the collision. Taking account of the absence of orbital magnetism of a free electron, not subject to a magnetic field, and $F_{c}(k)=1$ for a point charge, there is complete agreement between the results (7.10) and the sum of (12.2l) and (12.31).

The contribution (12.31) can be measured by forming the difference of data for $P_{2}=1$ and $P_{2}=-1$, since the contribution (12.21) will cancel in forming the difference. Keeping $\mathrm{P}_{2}$ fixed and switching the polarity of an external magnetic field, strong enough to saturate the magnetization, achieves the same result provided the charge-magnetic terms in (12.21) are negligible.

The occurance of different geometric factors with the spin and orbital contributions to (12.31) offers the possibility to separately measure the two contributions. For example, if the magnetization is aligned along the scattering vector the orbital contribution will not participate in the diffraction event. However, it is unlikely that many Bragg reflections will be accessible. 


\section{Spectroscopy}

\subsection{Prologue}

Spectroscopic studies of materials is taken to include non-resonant inelastic scattering events. At low energics, around $10 \mathrm{eV}$, the observed spectrum would include the excitation of collective modes, and interband transitions (Callaway, 1991). The recent development of high resolution $x$-ray spectrometers, for the measurement of phonon dispersions, plasmon peaks, particle-hole continuum etc. is reviewed by Burkel (1991). Here, the focus is on the high energy part of the spectrum probed with hard $x$-rays. However, the formalism described in $\$ 13.2$ is not limited to the interpretation of the hard $x$-ray region.

Compton scattering studies of charge distributions in solids, reviewed by Cooper (1985), are interpreted in terms of the Compton profile (8.5). Platzman and Tzoar (1965) and Grotch et al. (I983) have developed the theory of spin-dependent Compton scattering from bound clectrons. The result in $\$ 13.3 .3$ corresponds to the leading-order expression provided by Grotch et al. (1983), who consider also higherorder processes that engage conclations between spin and momentum variables. The scattering events in question arise in the interlerence between charge and magnetic amplitude induced by circular polarization in the primary beam, i.e. the analogue of mixed charge-magnetic reflections in Bragg scattering.

Experiments on ferromagnetic iron and nickel, using polarized photons from an electron synchrotron source, have been reported by Cooper et al. (1988) and Timms et al. (1990)). Directional spin-weighted momentum profiles show features that do not appear in calculations based on the augmented plane wave method for the electronic structure of melals (Callaway 1991). The main discrepancy between the data and calculations occurs at small momentá, where calculated profiles are larger than profiles deduced from data. Cooper el al. (1992) have made careful studies to detect the presence in Compton scattering data of a contribution from orbital magnetism, which has been observed in dilfraction experiments. Their conclusion is that orbital magnetism does not contribute to the magnetic Compton profile extracted from charge-magnetic interference scattering, induced by circular polarization in the primary beam. A calculation reported in $\$ 13.3 .2$, which is based on a proven model of the Compton process, leads to a result for the orbital contribution to the crosssection that is consistent with the lindings published by Cooper et al, (1992).

\subsection{Cross-section}

The expression developed in this section includes circular polarization in the primary beam, $\mathbf{P}=\left(0, \mathrm{P}_{2},(0)\right.$, and magnclic contributions to leading-order in $\tau=\left(\mathrm{E} / \mathrm{mc}^{2}\right)$. The starling point is (3.16). Examination of (3.22) for the components 
of $\alpha$ and $\beta$ shows that $\alpha_{1}$ and 2 are proprotional to $\tau$ so the terms $\alpha_{1}{ }^{+} \alpha_{1}$ and $\alpha_{2}{ }^{+} \alpha_{2}$ are not included in our expression. Perhaps the most compact formalism is achieved with correlation functions introduced in $\$ 5$. Recall that the frequency change in scattering is denoted by $\omega=c\left(q-q^{\prime}\right)$ and the concomitant wave vector change $\mathbf{k}=\mathbf{q}-\mathbf{q}^{\prime}$. For ease of notation, the $\mathbf{k}$-dependence of quantities will not always be displayed, e.g. the charge variable $n(\mathbf{k})$, defined in (2.10), is often written as $\mathrm{n}$ in subsequent working. The partial differential cross-section is

$$
\begin{aligned}
& \frac{d^{2} \sigma}{d \Omega d E^{\prime}}=r_{e}^{2}\left(q^{\prime} / q\right)(1 / 2 \pi \hbar) \int_{-\infty}^{\infty} d t \exp (-i \omega t)\left\{<\alpha_{3}^{+} \alpha_{3}(t)+\beta^{+} \beta(t)>\right. \\
& \left.+P_{2}<\beta^{+} \alpha_{2}(t)+\alpha_{2}^{+} \beta(t)+i\left[\alpha_{3}^{+} \alpha_{1}(t)-\alpha_{1}^{+} \alpha_{3}(t)\right]>\right\}
\end{aligned}
$$

where the term proportional to $\mathrm{P}_{2}$ is magnetic in character in as much that it vanishes for $\tau \rightarrow 0$.

On using the expressions listed in (3.22) and developing results for correlation functions to leading-order in $\tau$, the dynamic quantity in (13.1) becomes,

$$
\begin{aligned}
& \frac{1}{2}\left(1+\cos ^{2} \theta\right)<n^{+} n(t)>+\frac{i \tau}{2}\left(\hat{\mathbf{q}} \times \hat{\mathbf{q}}^{\prime}\right) \cdot\left\{\cos \theta<\mathrm{n}^{+} \mathbf{Z}(t)-\mathbf{Z}^{+} n(t)>\right. \\
& \left.-\mathrm{a}(1+\cos \theta)<\mathrm{n}^{+} \mathbf{S}(t)-\mathbf{S}^{+} \mathrm{n}(\mathrm{t})>\right\} \\
& +\frac{\tau}{2} P_{2}\left\{\left(\hat{\mathbf{q}}(1-\mathrm{a}+(\cos \theta-a) \cos \theta)+\hat{\mathbf{q}}^{\prime}(3 a \cos \theta-2 \cos \theta-a)\right)\right. \\
& \left.\cdot<\mathrm{n}^{+} \mathbf{S}(t)+\mathbf{S}^{+} n(t)>+\left(\hat{\mathbf{q}}+\hat{\mathbf{q}}^{\prime} \cos \theta\right) \cdot<\mathrm{n}^{+} \mathbf{Z}(t)+\mathbf{Z}^{+} n(t)>\right\} .
\end{aligned}
$$

Here, $\mathrm{a}=\left(1+\mathrm{q}^{\prime} / \mathrm{q}\right) / 2$ and the spin and momentum operators $\mathbf{S}$ and $\mathbf{Z}$ are defined in (3.18) and (3.19). The first term in (13.2) is the pure charge contribution, and evaluated in $\$ 7$ for free particles.

In subsequent sections, the various correlation functions in (13.2) are evaluated in the Compton limit, discussed in $\$ 8$ for the particular case of charge scattering.

\subsection{Compton Limit}

The Compton limit is achieved with a high primary energy ( $40 \mathrm{keV})$ and large scattering vectors. As discussed in $\$ \S 2$ and 8 , the scattering process in this limit is dominated by incoherent events that involve individual particles. 


\subsubsection{Charge Scattering}

The compton limit of charge scattering is discussed in $\$ \$ 2,7$ and 8 and the corresponding response function is given in (7.7) and (8.5). Here, the same result is obtained using a method that involves a specific model of Compton scattering which has proved valuable in the interpretation of charge scattering data, and is taken as a basis for the following discussion of the orbital contribution to the inelastic crosssection.

The charge matrix element for a single electron evaluated with momentumspace wave functions (8.7) is

$$
<\mu^{\prime}|\exp (\mathrm{ik} \cdot \mathbf{R})| \mu>=\int \mathrm{d} \mathbf{t} \chi_{\mu^{\prime}}^{*}(\mathbf{k}+\mathbf{t}) \chi_{\mu}(\mathbf{t})
$$

where $\mathbf{t}$ is used as a wave vector variable to avoid possible confusion with the wave vectors $\mathbf{q}, \mathbf{q}^{\prime}$ for the primary and secondary photons. If the final state of the electron is in the continuum, a reasonable approximation to the real-space wave function is a plane wave labelled by a wave vector $\mathbf{Q}$ for which

$$
\chi_{\mu^{\prime}}(\mathbf{t})=\left((2 \pi)^{3} / \Omega\right)^{1 / 2} \delta(\mathbf{t}-\mathbf{Q})
$$

Using this expression in (13.3),

$$
\left|<\mu^{\prime}\right| \exp (\mathbf{i k} \cdot \mathbf{R})|\mu>|^{2}=\left((2 \pi)^{3} / \Omega\right)\left|\chi_{\mu}(\mathbf{Q}-\mathbf{k})\right|^{2}
$$

and on integraling over all final wave vectors $Q$ with a density $\left(\Omega /(2 \pi)^{3}\right)$,

$$
\sum_{\mu^{\prime}}\left|<\mu^{\prime}\right| \exp (\mathrm{ik}, \mathbf{R})|\mu>|^{2}=\int \mathrm{d} \mathbf{Q}\left|\chi_{\mu}(\mathbf{Q})\right|^{2} .
$$

To complete the connection with the earlier discussion of Compton scattering from charges it is necessary to average over the initial states which occur with a probability $\mathrm{p}_{\mu}$;

$$
\sum_{\mu \mu^{\prime}} \mathrm{p}_{\mu} \mid\left.\left\langle\mu^{\prime}\right| \exp (\text { ik. } \mathbf{R})|\mu\rangle\right|^{2}=\int \mathrm{d} \mathbf{Q} \sum_{\mu} \mathrm{p}_{\mu}\left|x_{\mu}(\mathbf{Q})\right|^{2}=\hbar^{3} \int \mathrm{d} \mathbf{Q} \rho(\mathbf{Q})
$$


where $\rho(\mathbf{Q})$ is the momentum distribution function defined in (8.3). The conservation of energy that accompanies the model of events Jeading to (13.5) is

$$
\hbar \omega-\mathrm{E}_{\mathrm{k}}-\hbar^{2}(\mathbf{k} \cdot \mathbf{Q}) / \mathrm{m}=0
$$

with $\mathrm{E}_{\mathrm{k}}=(\hbar \mathrm{k})^{2} / 2 \mathrm{~m}$. To arrive at this result, the potential energy difference between the initial and final states is neglected.

\subsubsection{Orbital Contribution}

Following the line of discussion given in the previous subsection, which led to the standard expression for the Compton profile for charge scattering derived by other methods in $\$ \$ 7$ and 8 , our discussion of the orbital contributions to $(13.2)$ is based on a study of the matrix elements in

$$
<\mu\left|\mathrm{n}^{+}(\mathbf{k})\right| \mu^{\prime}><\mu^{\prime}|\mathbf{Z}(\mathbf{k})| \mu>
$$

evaluated for a single electron. The charge matrix element is given by (13.3). The corresponding result for $\mathbf{Z}$, in the limit of a single electron, is

$$
<\mu^{\prime}|\mathbf{Z}| \mu>\rightarrow(\mathrm{i} / \mathrm{q})\left(\hat{\mathbf{q}}-\hat{\mathbf{q}}^{\prime}\right) \times \int d \mathbf{t} \mathbf{t} \chi_{\mu^{\prime}}^{*}(\mathbf{k}+\mathbf{t}) \chi_{\mu}(\mathbf{t}) .
$$

If a plane wave is used to describe the final state of the electron,

$$
<\mu^{\prime}|\mathrm{n}(\mathbf{k})| \mu>^{*}<\mu^{\prime}|\mathbf{Z}(\mathbf{k})| \mu>\rightarrow\left|\chi_{\mu}(\mathbf{Q}-\mathbf{k})\right|^{2}(\mathrm{i} / \mathrm{q})\left(\hat{\mathbf{q}}-\hat{\mathbf{q}}^{\prime}\right) \times(\mathbf{Q}-\mathbf{k})
$$

A result of this calculation is that, with the chosen model of the Compton process, the product of matrix elements is purely imaginary.

The orbital contribution to (13.2) which is prefaced by $\mathrm{P}_{2}$ vanishes in the Compton limit, since the total combination of matrix elements is,

$$
\begin{gathered}
\left(<\mu\left|\mathrm{n}^{+}\right| \mu^{\prime}><\mu^{\prime}|\mathbf{Z}| \mu>+<\mu\left|\mathbf{Z}^{+}\right| \mu^{\prime}>\left\langle\mu^{\prime}|\mathrm{n}| \mu>\right)\right. \\
=2 \operatorname{Re} \cdot\left(<\mu\left|\mathrm{n}^{+}\right| \mu^{\prime}><\mu^{\prime}|\mathbf{Z}| \mu>\right)=0 .
\end{gathered}
$$

The other orbital contribution to (13.2) is, 
2i $\sum_{\mu^{\prime}} \operatorname{Im} .\left(<\mu\left|\mathrm{n}^{+}\right| \mu^{\prime}>\left\langle\mu^{\prime}|\mathbf{Z}| \mu \lambda=(2 \mathrm{i} / \mathrm{q})\left(\hat{\mathbf{q}}-\hat{\mathbf{q}}^{\prime}\right) \times \int \mathrm{d} \mathbf{Q}\left|\chi_{\mu}(\mathbf{Q})\right|^{2} \mathbf{Q}\right.\right.$.

Hence, the only contribution to the cross-section made by the orbital operator $\mathbf{Z}$ is,

$-\left(\mathrm{q}^{\prime} / \mathrm{q}\right)\left(\tau \mathrm{r}_{\mathrm{e}}^{2} / \mathrm{q}\right) \cos \theta(1-\cos \theta) \int d \mathbf{Q}\left|\chi_{\mu}(\mathbf{Q})\right|^{2} \mathbf{Q} \cdot\left(\hat{\mathbf{q}}+\hat{\mathrm{q}}^{\prime}\right) \delta\left(\hbar \omega-E_{\mathrm{k}}-\hbar^{2} \mathbf{k} \cdot \mathbf{Q} / \mathrm{m}\right)$.

After averaging it over the initial states, as described in (13.5), (13.8) is added to the standard Compton profile (8.5). Some physical insight for the result (13.8) is obtained by examining the exact cross-section for a single electron given in \$7. The leading correction to the classical non-relativistic result, in an expansion in $\tau$, is a term with the same structure as (13.8).

The integral in (13.8) can be estimated quite easily. Because Compton scattering is only mildly inelastic the vector $\left(\hat{\mathbf{q}}+\hat{\mathbf{q}}^{\prime}\right)$ is orthogonal to $\mathbf{k}$, to a good approximation, so that scalar product $\mathbf{Q} \cdot\left(\hat{\mathbf{q}}+\hat{\mathbf{q}}^{\prime}\right)$ can be written in terms of the argument of the delta-function. Let $Q_{0}=m\left(\hbar \omega-E_{k}\right) / \hbar^{2} k$, then

$$
\begin{gathered}
\int \mathrm{d} \mathbf{Q}\left\{\mathbf{Q} \cdot\left(\hat{\mathbf{q}}+\hat{\mathbf{q}}^{\prime}\right)\right\}\left|\chi_{\mu}(\mathbf{Q})\right|^{2} \delta\left(\hbar \omega-E_{\mathrm{k}}-\hbar^{2} \mathbf{k} \cdot \mathbf{Q} / \mathbf{m}\right) \\
\approx\left(\pi \mathrm{k} \cos \left(\frac{1}{2} \theta\right) / \mathrm{E}_{\mathrm{k}}\right) \int_{\| \prime}^{\infty} \mathrm{d} \rho^{2}\left|\chi_{\mu}(\mathrm{Q})\right|^{2} \rho
\end{gathered}
$$

where $Q^{2}=Q_{0}{ }^{2}+\rho^{2}$, If,

$$
\left|\chi_{\mu}(\mathbf{Q})\right|^{2} \propto \exp \left(-Q^{2} R_{a}^{2}\right)
$$

the ratio of (13.8) to the standard charge Compton protile is,

$$
\left\{\frac{-\tau \sin 2 \theta(1-\cos \theta) \pi^{\frac{1}{2}}}{\left(k R_{0}\right)\left(1+\cos ^{2} \theta\right)}\right\}
$$

It is interesting to observe that this ratio is small, because $\tau \ll<1$ and $\left(k R_{o}\right) \gg 1$, and it vanishes in the backward scattering geometry usually adopted in Compton scattering experiments, c.f, Azároff et al. (1974), Cooper (1985). 


\subsubsection{Spin Contribution}

Experiments by Cooper et al. (1992) have shown that Compton scattering from magnetic materials measure the spin densily. Their finding is supported by the model calculation reported in $\$ 13.3 .2$. This subsection addresses the structure of the spin correlation functions in (13.2) evaluated in the Compton limit.

To this end, we argue that the Compton limit of the mixed correlation function $\left\langle\mathrm{n}^{+} \mathrm{S}\right\rangle$ is

$\left.\left.<\mathrm{n}^{+}(\mathbf{k}) \mathbf{S}(\mathbf{k}, \mathrm{t})\right\rangle \approx \hbar^{3} \int \mathrm{d} \mathbf{Q} \sum_{j}<\delta\left(\hbar \mathbf{Q}-\mathbf{p}_{\mathrm{j}}\right) \mathrm{s}_{\mathrm{j}}>\exp \left\{\mathrm{it}\left(\mathrm{E}_{\mathrm{k}}+\hbar^{2} \mathbf{k} \cdot \mathbf{Q} / \mathrm{m}\right) / \hbar\right)\right\}$

On taking the time Fourier transform of this result, to obtain the appropriate response function, the exponential term produces a delta function which expresses conservation of energy, c.f. (13.16) and (8.5). An argument which produces the estimate (13.10) rests on the use of $\mathbf{R}(t)=(\mathbf{R}(0)+t \mathrm{p} / \mathrm{m})$ where $\mathrm{p}$ is the momentum conjugate to $\mathbf{R}=\mathbf{R}(0)$; this expression for $\mathbf{R}(\mathrm{l})$ is manifestly a short-time expansion. The first few steps leading to (13.10) are.

$$
\begin{aligned}
& \left\langle\mathrm{n}^{+}(\mathrm{k}) \mathrm{S}(\mathrm{k}, \mathrm{l})\right\rangle=\sum_{\mathrm{jj^{ \prime }}}\left\langle\exp \left(-\mathrm{ik} \cdot \mathbf{R}_{\mathrm{j}^{\prime}}\right) \exp \left(\mathrm{ik}, \mathbf{R}_{\mathrm{j}}(\mathrm{t})\right) \mathrm{s}_{\mathrm{j}}(\mathrm{t})\right\rangle \\
& \left.\approx \sum_{\mathrm{i}}<\exp \left(-\mathrm{ik} \cdot \mathbf{R}_{\mathrm{j}}\right) \exp \left\{\mathbf{i k} \cdot\left(\mathbf{R}_{\mathrm{j}}+\mathrm{t} \mathbf{p}_{\mathrm{j}} / \mathrm{m}\right)\right\} \mathbf{s}_{\mathrm{j}}(\mathrm{t})\right\rangle \\
& =\sum_{i}<\exp \left\{i t k \cdot p_{j} / m+i t E_{k} / \hbar\right\} s_{j}(t)>
\end{aligned}
$$

where the last line is reached by using a standard identity for the product of two exponential operators. Note that, just the self part of the correlation function is retained, i.e. the incoherent approximation is made. The final step from this last result to (13.10) entails some of the work reported in $\$ 8$.

Before collecting the results for the partial differential cross-section in the Compton limit, let us remark that in the extreme limit $\mathrm{E} \rightarrow \infty$ scattering is elastic, and the scattered intensity is proportional to the static limit of the correlation function, c.f. $\S 5$. The static limit of $\langle\mathrm{n}+\mathrm{S}\rangle$ in the incoherent approximation is simply the average value of the spin density $<\mathrm{S}>$. 
In subsequent working, the static correlation function in (13.10) is assumed to be purely real, in which case the polarization-independent spin contribution to (13.2) vanishes. Starting from (13.2), and gathering results from the foregoing discussions, the Compton limit of the cross-section is

$$
\begin{aligned}
\frac{\mathrm{d}^{2} \sigma}{\mathrm{d} \Omega \mathrm{dE} E^{\prime}}= & \hbar^{3}\left(\mathrm{q}^{\prime} / \mathrm{q}\right) \mathrm{r}_{\mathrm{e}}^{2}\left\{\frac{1}{2}\left(1+\cos ^{2} \theta\right) \int \mathrm{d} \mathbf{Q} \rho(\mathbf{Q}) \delta(\text { Energy })\right. \\
& +\tau \mathrm{P}_{2}\left[\left(\hat{\mathbf{q}}(1-\mathrm{a}+(\cos \theta-\mathrm{a}) \cos \theta)+\hat{\mathbf{q}}^{\prime}(3 \mathrm{a} \cos \theta-2 \cos \theta-\mathrm{a})\right)\right. \\
& \left.\left.\cdot \int \mathrm{d} \mathbf{Q} \sum_{\mathrm{j}}<\delta\left(\hbar \mathbf{Q}-\mathbf{p}_{\mathrm{j}}\right) \mathbf{s}_{\mathrm{j}}>\delta(\text { Energy })\right]\right\}
\end{aligned}
$$

where

$$
\delta(\text { Energy })=\delta\left(\hbar \omega-E_{k}-\hbar^{2} \mathbf{k} \cdot \mathbf{Q} / \mathrm{m}\right)
$$

If the inelasticity is sufficiently small, $a=(1+q / q) / 2 \sim 1$ and the orientation factor in the polarization dependent term is identical with that found for the spin contribution to the diffraction cross-section given in (12.31). The orientation factor in (13.11) is not the same as the one derived from the Klein-Nishina formula. This result is attributed to the different models; the Klein-Nishina result is the exact cross-section for a free electron that is at rest before the collision, whereas (13.11) is derived from an approximate amplitude appropriate for bound electrons. The polarizationindependent contribution to the cross-section derived from the orbital operator is not included in (13.11) on the grounds that, the estimate in $\$ 13.3 .2$ shows it is negligible under the conditions which prevail in the Compton limit. 
In general, it is useful to consider photon scattering by magnetic materials in one of two regimes of the incident photon energy. These are the limit of high photon energies, when the incident photon energy lies above the excitation energy of an absorption edge, and near resonance, when the incident energy is in the vicinity of an absorption edge. The $\mathrm{L}$ absorption edges of the rare earth elements are in the range $6-10 \mathrm{keV}$, and the $\mathrm{M}$ absorption edges of the actinides are $3-5 \mathrm{keV}$. Set against these figures, for Compton scattering the incident photon energy is chosen to be in the hard $\mathrm{x}$-ray region, and typically $40-50 \mathrm{keV}$.

Here, and in $\$ 15$, we consider the resonant regime and discuss elastic magnetic resonance scattering, and spin-dependent photon absorption which is also called circular magnetic $x$-ray dichroism. The formal relation between absorption and scattering is the standard optical theorem that reveals proportionality between the absorption coefficient and the imaginary part of the forward scattering amplitude. Within a simple one-electron picture of electronic structure, in resonant scattering the incident photon promotes by a virtual transition an inner shell electron to an unoccupied orbital above the Fermi energy, which subsequently decays through the emission of an elastically scattered photon. The amplitude for resonant magnefic scattering then depends on the matrix elements which couple the initial state and the intermediate magnelic states allowed by the Pauli exclusion principle. Turning back to the explicit strueture of the scaltering amplitude equ (11.4) we recall that if contains charge, linear momentum and magnetic interaction operators. To date, the experimental data on resonant scattering and dichroism have been successfully interpreted in terms of the momentum interaction operation. In this instance, the magnetic character of the observed electron-photon events in magnetic materials stems entirely from the nature of the wave functions used to calculate the dipole, quadrupole, ...., matrix elements. This aspect of the physics, while well established in the context of the Fano effect and magneto-optical Kerr effect, had been overlooked in the context of photon scattering by magnetic materials. Instead, attention was drawn to the spin interaction operator in the scattering amplitude but resonant effects due to this operator, a relativistic correction to the charge operator, are much too small to explain observed effects. Hence, the following discussion is concerned with magnetic effects revealed in resonant scattering and dichroism affected by the linear momentum operator in the scattering amplitude. Expansion of the exponential in the momentum operator generates dipole, quadrupole, ...., operators discussed in $\$ 6$.

To fix ideas, consider the schematic atomic energy level diagram displayed in Fig (10). Only $\mathrm{K}$ and $\mathrm{L}$ core atomic initial states are indicated together with some higher energy partially filled intermediatc states. The diagram illustrates an atom with a net magnetic moment on account of the excess of spin-up electrons. The core states will in reality have a finite energy width although this is not included in the diagram. 
If photoabsorption proceeds through the dipole operator the selection rule for the initial $(\ell)$ and final $\left(\ell^{\prime}\right)$ orbital angular momenta is $\ell^{\prime}=\ell \pm 1$. Thus, for the illustrated case of photoabsorption by a $2 p_{3 / 2}$ state, separated in energy from $2 p_{1 / 2}$ primarily by the spin-orbit interaction, the final spin-down states are of d-like character.

In the adsorption of circularly polarized photons, the projection (m) of the photoelectron angular momentum relative to the photon beam direction is changed by $\Delta m= \pm 1$. The spin direction of the electron, coupled to the angular momentum by the spinorbit interaction, is unchanged in photoabsorption.

Dichroism is measured by detecting the difference of the absorption coefficient of circularly polarized photons for parallel and antiparallel orientation of the photon spin and the net moment of the absorber, in a ferro-or ferrimagnetic state. The matrix elements in the absorption coefficient, calculated by use of Fermi's Golden Rule for transition rates, depend on the initial and final state spin-orbit configurations. By way of an example, consider the magneto-absorption for $\mathrm{p}_{1 / 2} \rightarrow \mathrm{d}$ and $\mathrm{p}_{3 / 2} \rightarrow \mathrm{d}$ calculated with a simple atomic model. It is found that, the difference in absorption coefficients for right and left-handed circular polarization $(\Delta \mu)$ for these Iwo transitions is of the same magnilude but opposite sign. Very strong dichroism effects (up to $20 \%$ of the absorption cross-section) have been observed in the $3 \mathrm{~d}-4 \mathrm{f}$ absorption spectra of rare-earth materials.

Two key physical quantities entering $\Delta \mu$ are the polarization of the photoelectron $\mathrm{P}_{\mathrm{e}}$, and the spin density available in the intermediale states, $\Delta \rho$. Indeed, Schütz et al. (1988) argue that

$$
\Delta \mu-\mathrm{P}_{\mathrm{c}} \Delta \rho
$$

Values of $\mathrm{P}_{\mathrm{e}}$ depend very much on the spin-orbil configuration of the initial state. An analysis of $\mathrm{L}$ absorption by a free Gd atom revealed that, on averaging over all final states, $\mathrm{P}_{\mathrm{e}}$ for absorption of a right circularly polarized photon is $\mathrm{P}_{\mathrm{e}}\left(\mathrm{L}_{1}\right)=-0.15, \mathrm{P}_{\mathrm{e}}\left(\mathrm{L}_{2}\right)=-0.45$ and $\mathrm{P}_{\mathrm{e}}\left(\mathrm{L}_{3}\right)=0.2$. The calculations also demonstrate that in the $\mathrm{L}_{2}$ and $\mathrm{L}_{3}$ absorption processes the transitions to $\mathrm{s}$-like final states have a very small probability; $\mathrm{L}_{2}$ absorption is thus mainly $2 p_{1 / 2} \rightarrow d_{3 / 2}$, and for $L_{3}$ the strongest transition is $2 p_{3 / 2} \rightarrow d_{5 / 2}$. In consequence, for this model system, the observed values of $\Delta \mu$ for $\mathrm{L}_{2}$ and $\mathrm{L}_{3}$ absorption should provide, respectively, information on $\Delta \rho\left(\mathrm{d}_{3 / 2}\right)$ and $\Delta \rho\left(\mathrm{d}_{5 / 2}\right)$.

Experimental data and theorclical results for $\mathrm{Gd} \mathrm{L}_{1}, \mathrm{~L}_{2}$ and $\mathrm{L}_{3}$ absorption and corresponding spin-dependent profiles are displayed in Fig. (11). The calculations are based on a single-electron band structure model, and they account for the experimental data on the $\mathrm{L}_{1}$ edge and the $\mathrm{L}_{2}, \mathrm{~L}_{3}$ absorption for positive encrgies. The experimental $\mathrm{L}_{2}, \mathrm{~L}_{3}$ spectra show a significant decrease of the $\mathrm{L}_{2}, \mathrm{~L}_{3}$ dichroism signal at the lower energy side, 
especially for the $\mathrm{L}_{3}$ edge. It has been proposed (Carra et al., 1991) that the discrepancy can be accounted for by atomic quadrupole transitions to empty $4 \mathrm{f}$ states, which in the case of $\mathrm{Gd}$ are negative spin polarized, i.e. the observed dichroism entails transitions (E1) to the conduction bands as well as transitions (E2) to the highly localized $4 \mathrm{f}$ states. Confirmation of this explanation could come from a study of the angular dependence of the absorption coefficient, since it is different for E1 and E2 transitions; the appropriate expressions are given by (14.19) and (14.20).

A novel form of circular dichroism has been proposed for experimental investigation by Strange et al. (1991). They point out that dichroism in photon spectra can be studied in fluorescence experiments in which the incident beam is circularly polarized, but only the frequency of the secondary photons is detected. The effect is demonstrated in a model calculation for iron based on a fully relativistic multiple-scattering theory, and findings support the view that the proposed experiment could be a good probe of metallic magnetism.

\subsection{Circular Dichroism}

A derivation of the absorption coefficient for circular dichroism is given for two main reasons. First, the derivation makes clear the nature of the physical processes and the standard approximations. Secondly, en route we obtain an expression for the scattering amplitude that is useful in a subsequent discussion of elastic resonant scattering. Hence, the working demonstrates a close relation between dichroism and resonant scattering that, at the outset, physical intuition leads us to expect.

To keep the mathematical features of the discussion to a minimum, we consider a simple physical model in which one electron in a core orbital, labelled by $\mu$, is promoted to a single intermediate orbital, $\eta$, after absorption of a photon of energy $E=\hbar \mathrm{cq}$. A more realistic model contains many intermediate states individually weighted by the probability that they are vacant in the atomic ground state, c.f. (14.8). However, the one electron model is a standard approximation. The intermediate orbital has a total decay width $\Gamma$ (of the order of a few $\mathrm{eV}$ for $\mathrm{L}$ edges in rare earth ions) due to both radiative and nonradiative (Auger and Coster-Kronig) processes, and it consists of the initial configuration with a hole in a core level and an additional electron in a higher energy level $\left(E_{\eta}>E_{\mu}\right)$.

The basis of the calculation is the following relation between the absorption coefficient $\mu(\mathrm{E})$ and the imaginary part of the resonant scattering amplitude evaluated in the forward direction, $f$, obtained from (11.14), namely,

$$
\mu(E)=(-4 \pi / q) \operatorname{Im} \cdot f(E)
$$

Turning to (11.4), if the atom is initially in the ground state, the resonant part of the forward scattering amplitude $\left(\mathbf{q}=\mathbf{q}^{\prime}\right)$ is, 


$$
\mathrm{f}(\mathrm{E})=\left(\mathrm{r}_{\mathrm{e}} / \mathrm{m}\right)\left\{\boldsymbol{\varepsilon}^{\prime} \cdot \mathbf{J}(-\mathbf{q})\left(\mathrm{E}_{\mu}+\mathrm{E}-\mathcal{H}\right)^{-1} \boldsymbol{\varepsilon} \cdot \mathbf{J}(\mathbf{q})\right\}
$$

where $\mathcal{H}$ is the atomic Hamiltonian, and $\mathbf{J}(\mathbf{q})$ is the spatial Fourier transform of the linear momentum and spin densities defined in (11.2). Note that $f(E)$ and $\mu(E)$ have the dimensions of length and area, respectively.

In truth, $f(E)$ defined above is, of course, a quantum mechanical operator and appropriate eigenstates of $\mathcal{H}$ must be inserted. A complete set of states of $\mathcal{H}$ is inserted, as shown in (9.11), one of which, $\eta$, is assumed to dominate the absorption event. Thus, the amplitude of interest is,

$$
\mathrm{f}(\mathrm{E})=\left(\mathrm{r}_{\mathrm{e}} / \mathrm{m}\right)\left\{\frac{\left\langle\mu\left|\boldsymbol{\varepsilon}^{\prime} \cdot \mathbf{J}(-\mathbf{q})\right| \eta\right\rangle\langle\eta|\boldsymbol{\varepsilon} \cdot \mathbf{J}(\mathbf{q})| \mu\rangle}{\left(\mathrm{E}_{\mu}+\mathrm{E}-\mathrm{E}_{\eta}+\mathrm{i} \Gamma / 2\right)}\right\}
$$

The task at hand now is to study the structure of the matrix elements in the numerator, and to identify the components observed with circular polarization.

First, looking at the definition given in (11.2), the matrix element of the spin operator in $\mathbf{J}(\mathbf{q})$ is assumed to be small compared with the matrix element of the linear momentum operator, p. Also, we assume that $\langle\eta|\mathbf{p}| \mu\rangle$ is finite, i.e. the virtual transition between the initial core state and the intermediate state is dipole-allowed. Following the steps in (6.13), and noting that at resonance $\mathrm{E}=\mathrm{E}_{\eta}-\mathrm{E}_{\mu}$,

$$
\hbar<\eta|\mathbf{p}| \mu>=\operatorname{imE}<\eta|\mathbf{R}| \mu>
$$

whereupon,

$$
\mathrm{f}(\mathrm{E})=(\mathrm{eq})^{2}\left\{\frac{\left\langle\mu\left|\boldsymbol{E}^{\prime} \cdot \mathbf{R}\right| \eta\right\rangle\langle\eta|\boldsymbol{\varepsilon} \cdot \mathbf{R}| \mu\rangle}{\left(\mathrm{E}_{\mu}+\mathrm{E}-\mathrm{E}_{\eta}+\mathrm{i} \Gamma / 2\right)}\right\}
$$

This expression is the dipole (E1) approximation to the resonant scattering amplitude for a single electron.

Circular dichroism corresponds to the imaginary part of $f(E)$ evaluated for a photon beam that contains circular polarization. Referring to $\$ 3.2$, we find the appropriate value for $\mathrm{f}(\mathrm{E})$ by averaging the right-hand side of (14.3) with respect to the polarization distribution described by the density matrix $(3,12)$. In consequence, we are led to consider the quantity, 


$$
\operatorname{Tr} .\left(\frac{1}{2}\{g+\mathbf{P} \cdot \boldsymbol{\sigma}\}<\mu\left|\boldsymbol{E}^{\prime} \cdot \mathbf{R}\right| \eta\right\rangle\langle\eta|\boldsymbol{\varepsilon} \cdot \mathbf{R}| \mu>)
$$

where the trace operation is taken with respect to the photon polarization states. As in (3.13), we write the dipole matrix element product as a linear combination of the unit matrix and Pauli matrices, $\sigma$, namely,

$$
\left\langle\mu\left|\mathbf{E}^{\prime} \cdot \mathbf{R}\right| \eta>\langle\eta|\boldsymbol{\varepsilon} \cdot \mathbf{R}| \mu>=\beta \rho+\alpha \cdot \boldsymbol{\sigma} .\right.
$$

Using $(\alpha, \beta=\mathrm{x}, \mathrm{y}, \mathrm{z})$,

$$
\operatorname{Tr}, \vartheta=2, \quad \operatorname{Tr} . \sigma_{\alpha}=0, \quad \operatorname{Tr} . \sigma_{\alpha} \sigma_{\beta}=2 \delta_{\alpha, \beta}
$$

leads readily to the required result,

$$
\operatorname{Tr} .\left(\frac{1}{2}\{\vartheta+\mathbf{P} \cdot \sigma\}\{\beta \vartheta+\alpha \cdot \sigma\}\right)=(\beta+\mathbf{P} \cdot \alpha) \text {. }
$$

Now, circular polarization is described by $P_{2}$, so we choose a Stokes vector $\mathbf{P}=\left(0, P_{2}, 0\right)$. The appropriate expression for $\mathrm{f}(\mathrm{E})$, obtained from (14.3) averaged over the polarization states, is then,

$$
f(E)=(e q)^{2}\left\{\frac{\left(\beta+P \alpha_{2}\right)}{\left(E_{\mu}+E-E E_{\eta}+i \Gamma / 2\right)}\right\} .
$$

The final step in the calculation of the absorption coefficient for circular dichroism is to determine $\alpha_{2}$, defined through (14.4); we do not need to determine $\beta$ because it cancels when we form the difference of $\mathrm{f}(\mathrm{E})$ evaluated for right and left-hand circular polarizations $\left(\mathrm{P}_{2}= \pm 1\right)$,

For the calculation of $\alpha_{2}$ it is convenient to use the notation,

$$
\mathbf{Q}=\langle\eta|\mathbf{R}| \mu\rangle
$$

in terms of which

$$
\mathbf{Q}^{*}=\langle\mu|\mathbf{R}| \eta\rangle \text {. }
$$

With this notation, (14.4) reads

$$
\left(\varepsilon^{\prime} \cdot Q^{*}\right)(\varepsilon \cdot \mathbf{Q})=\beta \delta+\alpha \cdot \sigma
$$


The left-hand side is represented as a $2 \times 2$ matrix using the representation given in Fig. (5). On examining (3.13), we find that $\alpha_{2}$, required in (14.5), is determined by a knowledge of the off-diagonal matrix elements, viz.

$$
\alpha_{2}=(i / 2)\left\{\left(\varepsilon_{\perp}^{\prime} \cdot \mathbf{Q}^{*}\right)\left(\varepsilon_{/ /} \cdot \mathbf{Q}\right)-\left(\boldsymbol{\varepsilon}_{/ /}^{\prime} \cdot \mathbf{Q}^{*}\right)\left(\boldsymbol{\varepsilon}_{\perp} \cdot \mathbf{Q}\right)\right\} .
$$

Let the $\mathrm{z}$ - $\mathrm{y}$ plane be defined by $\mathbf{q}$ and $\mathbf{q}^{\prime}$, and choose $\boldsymbol{\varepsilon}_{\perp}=\boldsymbol{\varepsilon}_{\perp}^{\prime}=(1,0,0)$. If $\mathbf{q}$ makes an angle $\varphi$ with respect to the $\mathrm{z}$ - axis,

$$
\varepsilon_{/ /}=(0, \cos \varphi,-\sin \varphi)
$$

Because we require the forward scattering amplitude $\varepsilon_{/ /}^{\prime}=\varepsilon_{/ /}$. Assembling the various components yields the key result,

$$
\alpha_{2}=\operatorname{Im} \cdot\left\{\mathbf{Q}_{\mathrm{y}}^{*} \mathbf{Q}_{\mathrm{x}} \cos \varphi+\mathbf{Q}_{\mathrm{x}}^{*} \mathbf{Q}_{\mathrm{z}} \sin \varphi\right\} .
$$

To conclude the derivation, from (14.1) and (14.5) the difference in the absorption coefficient $\Delta \mu(\mathrm{E})$ for right and left-hand circular polarization is,

$$
\begin{aligned}
\Delta \mu(\mathrm{E}) & =(-4 \pi / \mathrm{q})\left\{\operatorname{Im} \cdot \mathrm{f}\left(\mathrm{E}, \mathrm{P}_{2}=1\right)-\operatorname{Im} \cdot \mathrm{f}\left(\mathrm{E}, \mathrm{P}_{2}=-1\right)\right\} \\
& =8 \pi^{2} \mathrm{e}^{2} \mathrm{q} \alpha_{2} \delta\left(\mathrm{E}_{\mu}+\mathrm{E}-\mathrm{E}_{\eta}\right) .
\end{aligned}
$$

In reaching the second equality, the energy-conserving delta function arises in the limit $\Gamma \rightarrow 0$. Eqn (14.7), together with the definition (14.6), gives the value of the matrix elements in the limit of dipole (E1) transitions. The magnetic character of $\Delta \mu$ arises from the structure of the matrix elements, which we will examine in slightly more detail. An explicit magnetic component in $\Delta \mu$ arises from the spin density in $\mathbf{J}$ (q), c.f. (11.2) and (14.2), but in reaching (14.8) this component has been dropped in favour of a component of the Fourier transform of the linear momentum density.

Proceeding with a study of the dipole matrix element (14.6), let the initial state be characterized by total angular momentum J. A more complete notation is $|\mu\rangle=\mid \mathrm{JM} ; l>$ where $\mathrm{M}$ is the magnetic quantum number, $\mathrm{Jz}|\mu>=\mathrm{M}| \mu>$, and $\ell$ is the orbital angular momentum that defines a multiplet. Use of an orbital that contains just one $\mathbf{M}$ component is an assumption which would probably not be valid if a crystal field perturbation is significant, since such a perturbation usually generates orbitals that are linear combinations of several atomic states and thus with several M components (Weissbluth, 1978). If also $|\eta\rangle=\left|\mathrm{J}^{\prime} \mathrm{M}^{\prime} ; \ell^{\prime}\right\rangle$, with $l \neq \ell^{\prime}$ in the general case, the Wigner-Eckart theorem states that the 
geometric dependence of $\langle\eta|\mathbf{R}| \mu\rangle$ is contained in a $3 \mathrm{j}$ symbol. Following the phase convention used by Weissbluth (1978), for example, the result of interest is,

$$
\mathrm{Q}_{v}=\left\langle\eta\left|\mathrm{R}_{v}\right| \mu\right\rangle=(-1)^{\mathrm{J}^{\prime}-\mathrm{M}^{\prime}}\left(\begin{array}{lll}
\mathrm{J}^{\prime} & 1 & \mathrm{~J} \\
-\mathrm{M}^{\prime} & v & \mathrm{M}
\end{array}\right)\left(\mathrm{J}^{\prime} ; l^{\prime}\|\mathrm{R}\| \mathrm{J} ; l\right)
$$

where $v=0, \pm 1$ labels spherical components, and the right-hand side, apart from a phase factor, is the product of a $3 \mathrm{j}$ symbol and a reduced matrix element which in optical spectroscopy determines the line (oscillator) strength. Values of the reduced matrix element are obtained with a knowledge of the appropriate radial integrals and the steps involved are described, for example, by Cowan (1981).

The simplification to $\alpha_{2}$, defined in (14.7), brought about by using simple orbitals, that involve one $\mathrm{M}$ component, stems from a condition on the magnetic quantum numbers in the $3 \mathrm{j}$ symbol in (14.9); the $3 \mathrm{j}$ symbol is identically zero unless $\mathbf{M}^{\prime}=v+\mathrm{M}$. To see the impact of this condition on $\alpha_{2}$, consider the coefficient of sinq. The spherical components which occur in the Wigner-Eckart theorem are,

$$
Q_{0}=Q_{2}
$$

and

$$
\mathrm{Q}_{ \pm 1}=\mp \frac{1}{\sqrt{2}}\left(\mathrm{Q}_{\mathrm{x}} \pm \mathrm{i} \mathrm{Q}_{\mathrm{y}}\right)
$$

with

$$
Q_{v}^{*}=(-1)^{v} Q_{-v}
$$

Thus, $Q_{z}$ vanishes unless $M=M^{\prime} \pm 1$. In consequence, for orbitals that contain just one $M$ component, the product $\mathrm{Q}^{*}{ }_{\mathrm{X}} \mathrm{Q}_{2}=\mathrm{O}$, i.e. there is no term in $\alpha_{2}$ proportional to $\sin \varphi$. Similar reasoning leads to the result,

$$
\alpha_{2}=\frac{1}{2} \cos \varphi\left(|<\eta| R_{+1}|\alpha>|^{2}-|<\eta| R_{-1}|\alpha>|^{2}\right) .
$$

Hence, for this model of the absorption process, magnetic dichroism is proportional to the cosine of the angle between the direction of the photon beam and (magnetic) quantization axis, and the magnitude of $\Delta \mu(E)$ depends on the difference of the absolute values of $\left\langle\eta\left|\mathrm{R}_{ \pm 1}\right| \alpha\right\rangle$. The corresponding quadrupole contribution has a different dependence on $\varphi$, as well as different matrix elements.

In order to provide an explicit example of the dependence of $\Delta \mu(E)$ on the magnetic state of the atom, we pursue the calculation of $\alpha_{2}$ for a localized model of the magnetic properties of a rare earth magnet (Thole et al. 1985). Magnetic dichroism in the $\mathrm{M}_{4}$ and $\mathrm{M}_{5}$ 
absorption edge structure of rare earths leads to consideration of the transition $3 \mathrm{~d} \rightarrow 4 \mathrm{f}$. The initial state $3 \mathrm{~d}^{10} 4 \mathrm{f}^{\mathrm{n}}(\mathrm{J})$ is determined by Hund's rule. Dipole transitions are to the configuration $3 \mathrm{~d}^{9} 4 \mathrm{f}^{\mathrm{n}+1}\left(\mathrm{~J}^{\prime}\right)$ with $\mathrm{J}^{\prime}-\mathrm{J}=0, \pm 1$, c.f. (14.9); there are a plethora of states in the configuration that satisfy these selection rules, many of which will not be resolved in energy. Even so, in a study of linear dichroism taken up in the next section, Thole et al. (1985) correctly predicted that significant structure exists in the absorption spectrum (van der Laan et al, 1986).

Referring to the results (14.9) for the matrix elements that occur in $\alpha_{2}$ defined in (14.11), the $(2 \mathrm{~J}+1)$ degeneracy of the initial state is lifted in an ordered magnet by the Weiss molecular field. The interionic interaction responsible for this field couples pairs of ions at sites labelled by $\mathbf{m}$ and $\mathbf{n}$. If $\mathcal{S}(\mathbf{n})$ is the exchange interaction, defined to have the property $\ell(\mathbf{n}=0)=0$, the Heisenberg exchange interaction between spins is described by the Hamiltonian,

$$
\mathscr{H}=-\sum_{\mathrm{m}, \mathrm{n}} d(\mathbf{n}) \mathbf{S}_{\mathrm{m}} \cdot \mathbf{S}_{\mathrm{m}+\mathrm{n}}=-(\mathrm{g}-1)^{2} \sum_{\mathrm{m}, \mathrm{n}} d(\mathbf{n}) \mathbf{J}_{\mathrm{m}} \cdot \mathbf{J}_{\mathrm{m}+\mathrm{n}}
$$

where the second equality is valid when the exchange is smaller than the spin orbit splitting, and $g$ is the Lande factor. Within the molecular field approximation, the magnetic moment is,

$$
\langle\mathrm{J} Z\rangle=(1 / 2) \sum_{M=-J}^{J} M \exp (2 \mathrm{Mu})
$$

in which $\mathrm{Z}$ is the partition function, and

$$
\mathrm{u}=<\mathrm{J}^{\mathrm{Z}}>(\mathrm{g}-1)^{2} \sum_{\mathrm{n}} \mathrm{d}(\mathbf{n}) / \mathrm{k}_{\mathrm{B}} \mathrm{T} .
$$

The critical temperature $T_{c}$ satisfies,

$$
(\mathrm{g}-1)^{2} \sum_{\mathrm{n}} \mathrm{g}(\mathbf{n})=3 \mathrm{k}_{\mathrm{B}} \mathrm{T}_{\mathrm{c}} / 2 \mathrm{~J}(\mathrm{~J}+1) .
$$

From,

$$
Z=\sum_{M} \exp (2 \mathrm{Mu})=\sinh \{\mathrm{u}(2 \mathrm{~J}+1)\} / \sinh (\mathrm{u})
$$


it follows that,

$$
<\mathrm{JZ}>=(1 / 2 \mathrm{Z}) \frac{\mathrm{d}}{\mathrm{du}} \mathrm{Z}=\mathrm{JB}_{\mathrm{J}}(\mathrm{u})
$$

where the Brillouin function satisfies,

$$
2 \mathrm{JB}_{\mathrm{J}}(\mathrm{u})=(2 \mathrm{~J}+1) \operatorname{coth}\{\mathrm{u}(2 \mathrm{~J}+1)\}-\operatorname{coth}(\mathrm{u}) \text {. }
$$

From (14.9) and (14.11), the value of $\alpha_{2}$ thermally averaged over the initial states has the form,

$$
\alpha_{2}\left(\mathrm{~J}, \mathrm{~J}^{\prime}\right)=\gamma \mathrm{F}\left(\mathrm{J}, \mathrm{J}^{\prime}\right) \cos \varphi
$$

where $\gamma$ is proportional to the oscillator strength ( $\gamma$ depends on the quantum numbers but for simplicity these are not displayed) and

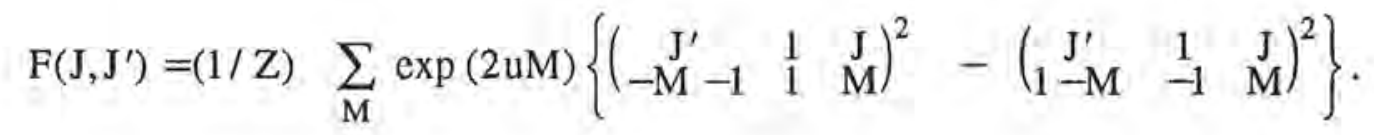

After some algebra one finds,

$$
\begin{aligned}
& \mathrm{F}(\mathrm{J}, \mathrm{J}-1)=-<\mathrm{JZ}>/ \mathrm{J}(2 \mathrm{~J}+1) \\
& \mathrm{F}(\mathrm{J}, \mathrm{J})=-<\mathrm{J} Z / J(J+1)(2 \mathrm{~J}+1)
\end{aligned}
$$

and

$$
\mathrm{F}(\mathrm{J}, \mathrm{J}+1)=\langle\mathrm{J} \mathrm{Z}>/(\mathrm{J}+1)(2 \mathrm{~J}+1)
$$

Hence, $\mathrm{F}\left(\mathrm{J}, \mathrm{J}^{\prime}\right)$ is proportional to the magnetic moment. The complete expression for $\Delta \mu$, which is the weighted sum of many terms proportional to $F\left(J, J^{\prime}\right)$, must reflect the thermodynamic properties of the rare earth magnet, e.g. $\Delta \mu$ continuously decreases as the temperature approaches $T_{c}$, and it vanishes in the paramagnetic state $T>T_{c}$.

We conclude this section by recording expressions for the matrix elements which are more general than those obtained so far, and also the expression used by Carra et al. (1991) in their study of E1 and E2 magnetic dichroism in gadolinium metal. Electrons participating in the absorption process are labelled by $\mathrm{j}$. The probability that the intermediate orbital $\eta$ is vacant for a given initial state $\mu$ is denoted by $\mathrm{p}_{\mu}(\eta)$, and the (Boltzmann) probability distribution for the initial states is denoted by $p_{\mu}$ (at absolute zero, $p_{\mu}=1$ ). Let $(L=1,2, \ldots$, and $-\mathrm{L} \leq \mathrm{M} \leq \mathrm{L}$ ) 


$$
f(E)=(e q)^{2} \quad\left\{\frac{(\beta+P . \alpha)}{\left(E_{\mu}+E-E_{\eta}+i \Gamma / 2\right)}\right\} .
$$

Synchrotron radiation is predominantly linearly polarized in the plane of the electron beam orbit, and described by the Stokes vector $\mathbf{P}=(0,0,-1)$. Following the notation used in (3.13), $\left(\beta-\alpha_{3}\right)$ is the diagonal element of the $2 \times 2$ matrix that is formed with initial and final polarization vectors which lie in the plane of scattering and denoted by $\boldsymbol{\varepsilon}_{/ /}^{\prime}$ and $\boldsymbol{\varepsilon}_{/ /}$; the explicit relation is,

$$
\left(\beta-\alpha_{3}\right)=\left(\varepsilon_{/ /}^{\prime} \cdot \mathbf{Q}^{*}\right)\left(\boldsymbol{\varepsilon}_{/ /} \cdot \mathbf{Q}\right),
$$

where $\mathbf{Q}$ is the dipole matrix element defined in (14.6). If the orbitals $|\mu\rangle$ and $|\eta\rangle$ are characterized by a single magnetic quantum number, as described in the text leading up to (14.9), then some dipole matrix elements vanish leaving

$$
\begin{aligned}
& \left(\beta-\alpha_{3}\right)=\left(\left|\mathrm{Q}_{\mathrm{y}}\right|^{2} \cos ^{2} \varphi+\left|\mathrm{Q}_{\mathrm{z}}\right|^{2} \sin ^{2} \varphi\right) \\
& =\frac{1}{2} \cos ^{2} \varphi\left\{|<\eta| \mathrm{R}_{+1}|\mu\rangle^{2}+|<\eta| \mathrm{R}_{-}|\mu\rangle^{2}\right\}+\left.\sin ^{2} \varphi|<\eta| \mathrm{R}_{0}|\mu\rangle\right|^{2},
\end{aligned}
$$

where $\varphi$ is the angle between the proton beam and (magnetic) quantization axis.

From (14.9) and using the notation adopted in (14.16), after averaging $\mathrm{Q}_{0}$ over the $(2 \mathrm{~J}+1)$ degeneracy of the initial state we find,

$$
|<\eta| \mathrm{R}_{0}|\mu>\rangle^{2}=\gamma \mathrm{F}_{\mathrm{o}}\left(\mathrm{J}, \mathrm{J}^{\prime}\right),
$$

where

$$
\mathrm{F}_{\mathrm{o}}\left(\mathrm{J}, \mathrm{J}^{\prime}\right)=(1 / \mathrm{Z}) \sum_{\mathrm{M}} \exp (2 \mathrm{uM})\left(\begin{array}{ccc}
\mathrm{J}^{\prime} & 1 & \mathrm{~J} \\
-\mathrm{M} & 0 & \mathrm{M}
\end{array}\right)^{2}
$$

Evaluating the $3 \mathrm{j}$ symbol leads to,

$$
\mathrm{F}_{\mathrm{o}}(\mathrm{J}, \mathrm{J}-1)=\left(\mathrm{J}^{2}-<\left(\mathrm{J}^{\mathrm{Z}}\right)^{2}>\right) / \mathrm{J}\left(4 \mathrm{~J}^{2}-1\right),
$$

and $F_{o}(J, J+1)$ is obtained from this result by making the substitution $\mathrm{J} \rightarrow(\mathrm{J}+1)$. Further algebra yields,

$$
\left\{|<\eta| \mathrm{R}_{H}|\mu>|^{2}+|<\eta| \mathrm{R}_{-1}|\mu>|^{2}\right\}=\gamma\left\{(1 /(2 \mathrm{~J}+1))-\mathrm{F}_{\mathrm{o}}\left(\mathrm{J}, \mathrm{J}^{\prime}\right)\right\} .
$$




$$
\begin{aligned}
& \mathrm{W}_{\mathrm{M}}^{\mathrm{L}}=q(2 \pi e)^{2}\left(\frac{\mathrm{L}+1}{\mathrm{~L}}\right) \sum_{\mu \eta} \mathrm{p}_{\mu} \mathrm{p}_{\mu}(\eta) \delta\left(\mathrm{E}_{\mu}+\mathrm{E}-\mathrm{E}_{\eta}\right) \\
& *|<\eta| \sum_{\mathrm{j}} \mathrm{j}_{\mathrm{L}}\left(\mathrm{qR}_{\mathrm{j}}\right) \mathrm{Y}_{\mathrm{M}}^{\mathrm{L}}\left(\hat{\mathbf{R}}_{\mathrm{j}}\right)|\mu>|^{2}
\end{aligned}
$$

where $\mathrm{j}_{\mathrm{L}}(\mathrm{x})$ is a spherical Bessel function and $\mathrm{Y}_{\mathrm{M}}^{\mathrm{L}}(\hat{\mathbf{R}})$ is a spherical harmonic (Weissbluth, 1978). For small arguments,

$$
\mathrm{j}_{\mathrm{L}}(\mathrm{x}) \sim \mathrm{x}^{\mathrm{L}} /(2 \mathrm{~L}+1) ! !
$$

The dipole $(\mathrm{L}=1)$ and quadrupole $(\mathrm{L}=2)$ expressions for circular dichroism (per atom) are,

$$
\Delta \mu^{(1)}=\left(6 \pi / \mathrm{q}^{2}\right) \cos \varphi\left\{\mathrm{W}_{1}^{1}-\mathrm{W}^{1}-1\right\}
$$

and

$$
\Delta \mu^{(2)}=\left(10 \pi / \mathrm{q}^{2}\right) \cos \varphi\left\{\left[\mathrm{W}_{2}^{2}-\mathrm{W}_{-2}^{2}\right] \sin ^{2} \varphi+\left[\mathrm{W}_{1}^{2}-\mathrm{W}_{-1}^{2}\right] \cos 2 \varphi\right\}
$$

Note that $\mathrm{W}_{\mathrm{M}}^{\mathrm{L}}$ is dimensionless. Using the small argument expansion of the Bessel function, it is quite easy to demonstrate that (14.19) is consistent with the previous result obtained for a simple model with single component initial and intermediate states with non-vanishing dipole matrix elements.

In their study of Gd, Carra et al. (1991) used relativistic Bloch functions to evaluate E1 matrix elements with 2 p core states. For comparison with experiment, they added a corehole lifetime (about $4 \mathrm{eV}$ ) and an energy-dependent intermediate-state lifetime. The calculation of the quadrupole spectra was made with an atomic model based on Hartree-Fock theory with relativistic corrections.

\subsection{Linear Dichroism}

In order to exhibit the salient features of linear dichroism obtained by Thole et al. (1985) and Goedkoop et al. (1988), we continue to use their model of the absorption process developed in $\S 14.1$. The starting point is the resonant scattering amplitude $f(E)$ averaged over the photon polarization states. From the discussion leading to (14.5), the appropriate expression is, 
for $\mathbf{J}^{\prime}=(\mathrm{J} \pm 1)$. Hence, for the chosen model, the thermodynamics revealed in linear dichroism appears solely in $\left\langle\left(\mathrm{J}^{2}\right)^{2}\right\rangle$ which appears in $\mathrm{F}_{\mathrm{o}}\left(\mathrm{J}, \mathrm{J}^{\prime}\right)$.

To complete the model calculation, observe that the absorption coefficient for linear dichroism is

$$
\mu_{\mathrm{o}}(\mathrm{E})=(-4 \pi / \mathrm{q}) \operatorname{Im} \cdot \mathrm{f}(\mathrm{E})=(2 \pi \mathrm{e})^{2} \mathrm{q}\left(\beta-\alpha_{3}\right) \delta\left(\mathrm{E}_{\mu}+\mathrm{E}-\mathrm{E}_{\eta}\right),
$$

with the factor $\left(\beta-\alpha_{3}\right)$ provided in (14.22),

We conclude with some remarks on the value of $\left\langle\left(\mathrm{J}^{\mathrm{z}}\right)^{2}\right\rangle$. In a pure paramagnet, the results

$$
<\left(\mathrm{J}^{\mathrm{Z}}\right)^{2}>=\frac{1}{3} \mathrm{~J}(\mathrm{~J}+1)
$$

follows because $\mathbf{J} \cdot \mathbf{J}=\mathrm{J}(\mathrm{J}+1)$. The corresponding value of $\mathrm{F}_{\mathrm{o}}(\mathrm{J}, \mathrm{J} \pm 1)$ is,

$$
\mathrm{F}_{\mathrm{o}}(\mathrm{J}, \mathrm{J} \pm 1)=1 / 3(2 \mathrm{~J}+1) \text {. }
$$

Inserting this value in (14.22) one finds that $\left(\beta-\alpha_{3}\right)$ is independent of $\varphi$, as it should be in the absence of a preferred magnetic axis. On the other hand, in the ordered state $\left(T \leq T_{c}\right)$ the molecular field approximation developed in $\$ 14.1$ provides the estimate

$$
<(\mathrm{J} Z)^{2}>=(1 / 4 \mathrm{Z}) \frac{\mathrm{d}^{2}}{\mathrm{du}^{2}} \mathrm{Z}=\mathrm{J}(\mathrm{J}+1)-<\mathrm{J} \mathrm{Z}>\operatorname{coth}(\mathrm{u})
$$

The result for $\left\langle\left(\mathrm{J}^{2}\right)^{2}\right\rangle$ is consistent with the standard identity for spin - $1 / 2$ operators $\left.<\left(\mathrm{J}^{\mathrm{z}}\right)^{2}\right\rangle=\left(\mathrm{J}^{\mathrm{z}}\right)^{2}=1 / 4$. In the limit $\mathrm{T} \rightarrow \mathrm{T}_{\mathrm{c}}, \mathrm{u} \rightarrow 0$ and from (14.25),

$$
<\left(\mathrm{J}^{\mathrm{Z}}\right)^{2}>\rightarrow \frac{1}{3} \mathrm{~J}(\mathrm{~J}+1)\left\{1+\frac{2}{15} \mathrm{u}^{2}(2 \mathrm{~J}-1)(2 \mathrm{~J}+3)\right\}=\frac{1}{3} \mathrm{~J}(\mathrm{~J}+1)\left\{1+\frac{(2 \mathrm{~J}+3)(2 \mathrm{~J}-1)}{\left(2 \mathrm{~J}^{2}+2 \mathrm{~J}+1\right)}\left(\frac{\mathrm{T}_{\mathrm{c}}-\mathrm{T}}{\mathrm{T}_{\mathrm{c}}}\right)\right\},
$$

where the second line is achieved with results provided in $\$ 14.1$. In the opposite extreme, $\mathrm{T} \rightarrow 0$, only one initial state is accessible and $\left\langle\left(\mathrm{J}^{\mathrm{z}}\right)^{2}\right\rangle=\mathrm{J}^{2}$. In this instance, $\mathrm{F}_{\mathrm{o}}(\mathrm{J}, \mathrm{J}-1)=0$ while,

$$
\mathrm{F}_{\mathrm{o}}(\mathrm{J}, \mathrm{J}+1)=1 /\{(\mathrm{J}+1)(2 \mathrm{~J}+3)\} \text {. }
$$


So, for $T=0$ and $J^{\prime}=(J-1)$, the matrix elements $\left(\beta-\alpha_{3}\right)$ are proportional to $\cos ^{2} \varphi$, while for $J^{\prime}=(J+1)$ it is a weighted sum of $\cos ^{2} \varphi$ and $\sin ^{2} \varphi$, although the angular dependence vanishes for $\mathrm{J}=1 / 2$.

Results calculated by Thole et al, (1985) for the $\mathrm{M}_{4,5}$ absorption spectra for Dy $4 \mathrm{f}^{9}$ $\left({ }^{6} \mathrm{H}_{15 / 2}\right)$ are shown in Fig. (12). The three curves demonstrate significant structure in the spectra for the extreme limits $\mathrm{T}=0$, and a pure paramagnet $(\mathrm{T} \rightarrow \infty)$. For $\mathrm{T}=0$, the results show marked differences between the spectra for $\varphi=0$ and $\varphi=\pi / 2$, which corresponds to polarization perpendicular $(\varphi=0)$ and parallel $(\varphi=\pi / 2)$ to the quantization axis. The authors observe that since the polarization dependence varies as $\left\langle\left(\mathrm{J}^{2}\right)^{2}\right\rangle$, the temperature dependence of the local moment can be determined in antiferromagnetic, ferrimagnetic and ferromagnetic systems.

In terms of the more general formalism surveyed at the end of $\$ 14.1$, the E1 absorption coefficient (14.24) is,

$$
\mu_{0}^{(1)}(\mathrm{E})=\left(6 \pi / \mathrm{q}^{2}\right)\left\{\frac{1}{2}\left[\mathrm{~W}_{1}^{1}+\mathrm{W}_{-1}^{1}\right] \cos ^{2} \varphi+\mathrm{W}_{0}^{1} \sin ^{2} \varphi\right\}
$$

This result describes the so-called white line in the absorption spectrum, and it is the companion to $\Delta \mu^{(1)}(\mathrm{E})$ for circular dichroism given in (14.19). In the appropriate limit, (14.26) is compatible with (14.22) and (14.24). 
When the incident photon energy is tuned near an absorption edge, large resonant enhancements of the scattering may be observed. The cross-section depends on the specific absorption edge, photon polarization states, and the magnetic state of the sample. The latter feature makes resonant scattering a potentially useful technique for the investigation of magnetic materials. The largest resonant enhancements have been observed for incident photon energies near the $M_{4}$ absorption edges in actinides, and near the $\mathrm{L}_{3}$ absorption edges in rare earth and transition metals. The first observation of resonant scattering was reported by Gibbs et al. (1988) in a study of the magnetic spiral structure of metallic holmium, and an interpretation in terms of electric multipole transitions followed from Hannon et al. (1988). An introduction to the ideas from Hannon et al. (1988) is given in $\$ 15.1$ following comments on some experiments which are more thoroughly discussed by Lander and Stirling (1992) and Stirling and Lander (1993).

The total coherent elastic amplitude for photon scattering is the sum of pure charge and pure non-resonant magnetic contributions, and a contribution from dispersive and absorptive processes. The latter contain both charge and magnetic interactions, as is evident from (11.4). In the experiments reported to date the observed intensities and polarization dependencics are accounted for by the charge (electric) contribution to resonant processes. In this case, the magnetic character of the scattering arises from the magnetic character of the electronic orbitals that enter the calculation of the matrix elements of the multipole operators. For holmium studied by Gibbs et al. (1988) and Gibbs et al. (1991), the significant events are virtual dipole - allowed transitions, coupling $2 \mathrm{p}$ core electrons with 5 d-derived conduction band states, and quadrupole transitions, coupling $2 \mathrm{p}$ core electrons to $4 \mathrm{f}$ atomic-like states. It is found for the spiral magnetic phase that some magnetic satellites arise solely from quadrupole transitions, while the remaining observed satellites are mixtures of dipolar and quadrupolar transitions.

The interpretation of resonant magnetic seattering provided by Hannon et al. (1988) accounts for the observations on holmium reported by Gibbs et al. (1991), and the findings of measurements on various uranium compounds with an antiferromagnetic configuration of moments (McWhan et al, 1990, Tang et al. 1992). Large resonant cross-sections observed at the $\mathrm{M}_{4}$ edge of $\mathrm{U}$ in UAs have been successfully interpreted in terms of three (electric) dipole operators that add coherently as demonstrated by the data displayed in Fig (13). It was confirmed from a polarization analysis of magnetic reflections that (with an incident beam $\sigma$ polarized) dipole transitions give contributions to the amplitude of $\sigma-\pi$ events which are linear in the magnet moment direction, leading to first-order magnetic reflections 
in the antiferromagnetic structure of UAs that occurs in the temperature range 63$127 \mathrm{~K}$.

The polarization dependence of resonant scattering, satellite selection rules, and basic features of line shapes are quite elementary consequences of a model based on electric multipole contributions to the resonant scattering amplitude. The nontrivial calculation required to confront experimental and theoretical findings is the calculation of radial integrals which arise in matrix elements of multipole operators. Where this has been attempted, ab initio calculations based on an atomic model have provided a good account of experimental data.

\subsection{Resonant Scattering Amplitude}

The description of elastic resonant scattering has much in common with dichroism, considered in $\$ 14$. Referring to eqn (14.2), the scattering amplitude operator is

$$
\mathrm{f}(\mathrm{E})=\left(\mathrm{r}_{\mathrm{e}} / \mathrm{m}\right) \quad\left\{\frac{\left\langle\mu\left|\boldsymbol{\varepsilon}^{\prime} \cdot \mathbf{J}\left(-\mathbf{q}^{\prime}\right)\right| \eta>\langle\eta|\varepsilon \cdot \mathrm{J}(\mathrm{q})| \mu>\right.}{\left(\mathrm{E}_{\mu}+\mathrm{E}-\mathrm{E}_{\eta}+i \Gamma / 2\right)}\right\}
$$

Here, the intermediate state, labelled $\eta$, is taken to be a single electronic orbital; formulae for a more general case are provided later in this section. Neglecting the contribution to $\mathbf{J}$ from the electron spin, and treating the contribution from the linear momentum in terms of the dipole approximation, $\mathrm{f}(\mathrm{E})$ does not depend on the wave vector of either the incident $(\mathbf{q})$ or secondary $\left(\mathbf{q}^{\prime}\right)$ photon. Near resonance, the amplitude (15.1) reduces to

$$
f(E)=(e q)^{2}\left\{\frac{\left(\varepsilon^{\prime} \cdot Q^{*}\right)(\varepsilon \cdot Q)}{\left(E_{\mu}+E-E_{\eta}+i \Gamma / 2\right)}\right\}
$$

where

$$
\mathbf{Q}=\langle\eta|\mathbf{R}| \mu\rangle \text {, }
$$

is a dipole matrix element.

Following the development pursued in $\$ 14$, we adopt a simple representation of the orbitals which reduces the product of matrix elements in (15.2) to an appealing form. Let the initial and intermediate orbitals be represented by states described by a single magnetic quantum number. In this instance, $Q^{*}{ }_{v} Q_{v^{\prime}}(\nu=0, \pm 1)$ vanishes unless the spherical component labels satisly the condition $\nu=\nu^{\prime}$, and so 


$$
\begin{aligned}
& \left(\varepsilon^{\prime} \cdot Q^{*}\right)(\varepsilon \cdot Q)=\sum_{v} \varepsilon_{v}^{\prime} \varepsilon_{v}^{*}\left|Q_{v}\right|^{2}=\frac{1}{2}\left\{\left(\varepsilon^{\prime} \cdot \varepsilon\right)\left(\left|Q_{+1}\right|^{2}+\left|Q_{-1}\right|^{2}\right)\right. \\
& \left.+\operatorname{im} .\left(\varepsilon^{\prime} \times \varepsilon\right)\left(\left|Q_{-1}\right|^{2}-\left|Q_{+1}\right|^{2}\right)+\left(\varepsilon^{\prime} \cdot m\right)(\varepsilon \cdot m)\left(2\left|Q_{0}\right|^{2}-\left|Q_{+1}\right|^{2}-\left|Q_{-1}\right|^{2}\right)\right\} .
\end{aligned}
$$

The three terms in the second equality are arranged in ascending powers of the (unit) vector $\mathbf{m}$ that defines the magnetic quantization axis. It comes as no surprise that, the combinations of dipole matrix elements in (15.3) arise also in the description of E1 circular and linear dichroism.

The dependence of the dipole matrix elements in (15.3) on the magnetic state of the target sample has been illustrated in $\$ 14$, and the discussion given there for dichroism is directly relevant to the present discussion of coherent scattering since the observed cross-section is proportional to the absolute square value of the thermally averaged scattering (length) amplitude. When it comes to consideration of the photon polarization states the quantity to be averaged is the cross-section, i.e. the absolute square of the total scattering amplitude. In forming the thermal average of the scattering amplitude there is need to consider both the magnetic effects, discussed in $\$ 14$, and the lattice vibrations. The latter are properly included by weighting the amplitude by Debye-Waller factors.

With regard to the dependence of the scattering amplitude on polarization states, the following representations of $\varepsilon^{\prime} . \varepsilon, \varepsilon^{\prime} \mathbf{x} \varepsilon$ and $\left(\varepsilon^{\prime} . \mathbf{m}\right)(\varepsilon . m)$ could be useful. The scattering geometry used in construction of the $2 \times 2$ matrices is detailed in Fig (6). The notation of $\sigma$ and $\pi$ polarization states appears in the literature; a photon polarized perpendicular to the plane of scattering is said to exhibit $\sigma$ polarization, while a photon polarized in the plane has $\pi$ polarization.

From Fig (14) we see that, for the scattering amplitude formed with (15.3) there are three forbidden events, namely, $\sigma-\pi$ events in the term which is independent of $\mathrm{m}$, and $\sigma$ - $\sigma$ events in the term proportional to $\mathrm{m}$. When the magnetic moment lies in the plane of scattering, $m_{\perp}=0$, the only event in the term of order $\mathrm{m}^{2}$ is $\pi-\pi$ scattering, while for $m_{\perp}=1$ the only event is $\sigma-\sigma$ scattering.

To illustrate the contribution made to Bragg intensities by resonant events, consider the term of order $\mathrm{m}$ in the resonant amplitude. Inspection of (15.3) shows that this term is of the form $\chi \cdot \mathbf{m}(\mathbf{R})$ where the vector $\mathbf{R}$ defines the position of the magnetic atom. For coherent scattering from an array of atoms the total amplitude is,

$$
\sum_{\mathbf{R}} \exp (\mathrm{ik} \cdot \mathbf{R}) \chi \cdot \mathbf{m}(\mathbf{R})
$$


where, as usual, $\mathbf{k}=\mathbf{q}-\mathbf{q}^{\prime}$. A simple magnetic configuration that gives rise to satellite reflections is a spiral structure in which the moments are perpendicular to an axis, and rotation about the axis varies sinusoidally with position along the axis. Such a spiral configuration of magnetic moments is realized in holmium in the temperature interval $20-132 \mathrm{~K}$. Let

$$
\mathbf{m}(\mathbf{R})=\mathrm{m}(0,0, \cos (\mathbf{w} \cdot \mathbf{R}))
$$

where the moment magnitude, $\mathrm{m}$, and moduation wave vector, $\mathbf{w}$ vary with temperature. The scattering amplitude is then of the form,

$$
\left(\frac{1}{2} \mathrm{~m} \chi\right) \sum_{\mathbf{k}}[\exp \{\mathrm{iR} \cdot(\mathbf{k}+\mathbf{w})\}+\exp \{\mathrm{iR} \cdot(\mathbf{k}-\mathbf{w})\}]
$$

from which we conclude that Bragg scattering occurs when

$$
k \pm \mathbf{w}=\tau
$$

where $r$ is a reciprocal vector for the magnetic latlice. Consideration of all three terms in the resonant amplitude shows that, dipole (E1) resonant scattering can contribute to a main Bragg reflection and satellites to it defined by $\mathbf{w}$ and $2 \mathbf{w}$, i.e. satellites of order 1 and 2 .

The E2 amplitude reported by Hannon et al. (1988) contains terms in $\mathbf{m}$ from zero up to fourth order, and there are thirteen distinct contributions. Hence, E2 resonant scattering can contribute to a main Bragg reflection and satellites to it of order 1 through 4 .

The model for E1 events used up to now in this section is based on single atomic orbitals for the initial and intermediate states. We conclude by recording the expression for the amplitude in which more than one initial and intermediate state is required. But, we retain the simplifying assumption that each orbital contains one $\mathrm{M}$ component, e.g. crystal-field perturbations are not included. The notation for the more general model follows that introduced in \$14. Let the dimensionless quantity,

$$
\begin{aligned}
\mathrm{F}_{\mathrm{M}}^{\mathrm{L}}(\mathrm{E}) & =\mathrm{q}(2 \pi \mathrm{e})^{2}\left(\frac{\mathrm{L}+1}{\mathrm{~L}}\right) \sum_{\mu \eta} \mathrm{p}_{\mu} \mathrm{p}_{\mu}(\eta)|<\eta| \sum_{\mathrm{j}} \mathrm{j}_{\mathrm{L}}\left(q R_{\mathrm{j}}\right) \mathrm{Y}_{\mathrm{M}}^{\mathrm{L}}\left(\hat{\mathbf{R}}_{\mathrm{j}}\right)|\mu\rangle^{2} \\
& /\left(\mathrm{E}_{\mu}+\mathrm{E}-\mathrm{E}_{\eta}+\mathrm{i} \Gamma / 2\right),
\end{aligned}
$$

in terms of which the El amplitude is, 


$$
\begin{aligned}
f(E)= & \left(\frac{3}{4 \pi q}\right)\left\{\left(\varepsilon^{\prime} \cdot \varepsilon\right)\left(\mathrm{F}_{+1}^{1}+F_{-1}^{1}\right)+\operatorname{im} \cdot\left(\varepsilon^{\prime} \times \varepsilon\right)\left(\mathrm{F}_{-1}^{1}-\mathrm{F}_{+1}^{1}\right)\right. \\
& \left.+\left(\varepsilon^{\prime} \cdot \mathrm{m}\right)(\varepsilon \cdot \mathrm{m})\left(2 \mathrm{~F}_{0}^{1}-\mathrm{F}_{+1}^{1}-\mathrm{F}_{-1}^{1}\right)\right\}
\end{aligned}
$$

The Debye-Waller factor is not shown explicitly in (15.5). The corresponding E2 amplitude contains linear combinations of $\mathrm{F}^{2} \mathrm{M}$. For a given magnetic reflection, the amplitude is the coherent sum of contributions from E1, E2, ... events. Line shapes, proportional to the absolute square of the total amplitude, as a function of photon energy can display marked asymmetries, which result from the interference of the resonant and non-resonant contributions.

Tang et al. (1991) reports ab initio atomic calculations of $\mathrm{F}^{1} \mathrm{M}(\mathrm{E})$ and $\mathrm{f}(\mathrm{E})$ at the $\mathrm{M}_{4}$ and $\mathrm{M}_{5}$ edges of uranium in $\mathrm{UO}_{2}\left(\mathrm{U}^{4+}\right)$ and $\mathrm{USb}\left(\mathrm{U}^{3+}\right)$. With the magnetic moments arranged to lic in the plane of scattering, the observed $\sigma-\pi$ scattering is proportional to the $\mathbf{m}$. $\left(\varepsilon^{\prime} \times \varepsilon\right)$ contribution to the amplitude (15.5). Radial integrals in $\mathrm{F}_{\mathrm{M}}^{1}$ (E) were obtained from a Hartnce-Fock scheme, including relativistic corrections. Fits to the experimental data are good for $\mathrm{UO}_{2}$, modelled by $\mathrm{U}^{4+}$, indicating that an atomic picture is uscful. Some discrepancies are found between experimental data and calculations for USb, which might indicate the possible need to go beyond an atomic picture and include hydridization between $\mathrm{f}$ states and band states. In the atomic picture the magnetic order is brought about by use of a magnetic field. 


\section{Concluding Remarks}

While there has been much experimental activity since 1985, a year made notable by the accomplishments of Gibbs et al. on structural modulations that accompany the magnetic ordering in holmium and Thole et al. who predicted a sizeable linear dichroism in magnetic matcrials, the measurement of magnetic properties of solids using photon beams is surely an inchoate subject. The recent spate of work has demonstrated the potential richness of the subject, although at present there are just a few examples of new science concerning basic magnetic features of solids. Future work might be driven by the few success stories, the remaining novelty, the operation of yet more photon factories, and developments in experimental methods, e.g. a method for the direct measurement of the degree of circular polarization in the secondary beam. A comparison with developments of neutron beam techniques to study magnetic materials, which began in earnest in the mid-50's and continues today, supports the view that it might be another decade before photon beam techniques can be placed in proper context with established techniques. 


\section{Achnowledgements}

Correspondence and discussions with the following researchers is gratefully acknowledged; F de Bergevin, S P Collins, M J Cooper, D Gibbs, M D Hamrick, G H Lander, S Manninen, D B McWhan, G. Priftis, G A Sawatzky, G T Trammell, K N Trohidou,

Figs. 7, 8, 11, 12 and 13 are reproduced with the consent of the authors, and the APS (Figs. 7, 8, 12 and 13) and SERC Daresbury Laboratory (Fig. 11). 


\section{Figure Captions}

(1) The main events induced by a photon beam impinging on a solid are shown in a schematic fashion.

(2) The two basic contributions to the Compton intensity are illustrated. The core and valence electron contributions are distinguished by the size of the Doppler broadening.

(3) Vectors and angles appropriate for Thomson scattering. In the text, one state of the polarization of the secondary photon $\boldsymbol{\varepsilon}^{\prime}(i)$ is taken to be in the plane perpendicular to the wave vector of the primary photon $q$. The second polarization state is described by $\varepsilon^{\prime}$ (ii) that lies in the plane of scattering. Hence, $\varepsilon^{\prime}(i) \times \varepsilon^{\prime}(i i)=\hat{\mathbf{q}}$.

(4) Coordinates $\xi, \eta, \zeta$ introduced in $\$ 3$ are illustrated. The $\xi$ axis is perpendicular to the plane of scattering defined by the primary and secondary photon wave vectors $q$ and $q^{\prime}, k=q-q^{\prime}$, and $\theta$ is the scattering angle. It is common practice to label $\varepsilon_{\perp}$ and $\varepsilon_{\perp}^{\prime}$ as $\sigma$-polarization states, and $\varepsilon_{/ /}$and $\varepsilon_{/ /}^{\prime}$ as $\pi$-polarization states.

(5) The representation chosen for the polarization-dependent elements of the scattering amplitude $G$ expressed as a $2 \times 2$ matrix eqn (3.13).

(6) The uppermost "seagull" space-time Feynman diagram depicts the $\mathrm{A}^{2}$ contribution to the generalized Kramers-Heisenberg formula. The lower two diagrams, with lime running from left to right, depict the first-order contributions from the p.A terms, and intermediate electron states are labelled $\bar{\mu}$.

(7) Two satellites observed in scattering from holmium at $17 \mathrm{~K}$. The filter isolates $(\sigma-\pi)$ events which are purely magnetic, After Gibbs et al. (1985)

(8) The solid square at $\theta=0$ corrcsponds to the degree of linear polatization of the primary beam. The open circles show the degree of linear polarization measured for the charge scattering at chemical Bragg reflections in holmium at a temperature where the magnetic configuration is conical $(\mathrm{T}<20 \mathrm{~K})$. The solid line is the degree of linear polarization for charge scattering calculated from eqn $(12,3)$ with $P_{3}=0.77$. The solid circles show the degree of linear polarization measured for magnetic (satcllite) reflections. The dashed lines are calculations based on results given in $\$ 12.4$ for three different values of 
the total spin (S) and total orbital angular (L) momentum. After Gibbs et al. (1991).

(9) The special choice for the $x, y, z$ coordinates used in $\$ 12$ and are illustrated. Magnetic quantization is parallel to the $\mathrm{z}$-axis, $\mathbf{k}=\mathbf{q}-\mathbf{q}^{\prime}$ and $\left(\hat{\boldsymbol{q}} \times \hat{\boldsymbol{q}}^{\prime}\right)$ is parallel with the $y$-axis which is aligned opposite to the $\xi$-axis. See also Fig (4).

(10) An illustration of electron states engaged in dichroism; only a few core states are displayed, and the conduction bands are shown without the rich structure that exists in results from a realistic model.

(11) Experimental (dashed line) and theoretical (solid line) absorption and corresponding absorption coefficient ( $\mu_{\mathrm{c}} / \mu_{\mathrm{o}}$ is a normalized value for $\Delta \mu$ in eq (14.8)) for $\mathrm{L}_{1}, \mathrm{~L}_{2}$ and $\mathrm{L}_{3}$ edges of gadolinium metal. After Schütz (1991).

(12) Calculated spectrum for linear dichroism of Dy (a) for $T>T_{C}$, and for $T=0$ with polarization (b) perpendicular and (c) parallel to the direction of the molccular field. After Thole et al. (1985).

(13) The energy dependence of the intensity at a satellite in UAs through the $\mathrm{M}_{5}$, $\mathrm{M}_{4}$ and $\mathrm{M}_{3}$ absorption edges. The solid line through the data is a fit to the coherent sum of three dipole oscillators. Inset is an expanded view of the $\mathrm{M}_{3}$ edge. After McWhan et al. (1990).

(14) $\hat{\mathbf{q}}$ and $\hat{\mathbf{q}}^{\prime}$ denote unit vectors in the directions of the primary and secondary photon beams (for elastic scattering $q=q^{\prime}$ ) and $\hat{\mathbf{q}} \cdot \hat{\mathbf{q}}^{\prime}=\cos \theta$ where $\theta$ is the angle of scattering. The vector $\mathbf{m}$ is a unit vector, which in $\$ 15$ defines the magnetic quantization axis, and $\mathrm{m}_{\perp}$ is the projection of $\mathbf{m}$ perpendicular to the plane of scattering. Polarization vectors parallel and perpendicular to the plane are denoled by $\varepsilon_{\perp}$ and $\varepsilon_{/ /}$, with $\mathrm{m}_{\perp}=\left(\varepsilon_{\perp}, \mathbf{m}\right)$. Note $\hat{\mathbf{q}}=\varepsilon_{\perp} \times \varepsilon_{/ /}$and a similar relation for the secondary beam. 


\section{REFERENCES}

Azároff, L.V., Kaplow, R., Kato, N., Weiss, R.J., Wilson, A.J.C., and Young, R.A. (1974), X-ray Diffraction (McGraw-Hill, N.Y.).

Balcar, E. and Lovesey, S.W. (1989) Theory of Magnetic Neutron and Photon Scattering (O.U.P.).

de Bergevin, F. and Brunel, M. (1981) Acta Cryst. A37, 314.

de Bergevin, F., and Brunel, M. (1986) Structure and Dynamics of Molecular Systems II, ed. R Daudel et al. (New York: Reidel)

Berestetskii, V.B., Lifshitz, E-M. and Pitaevskii, L.P. (1982) Quantum Electrodynamics, Course of Theoretical Physics Vol. 4 (Pergamon Press, Oxford).

Bhatt, G., Grotch, H., Kazes, E. and Owen, D.A. (1983) Phys.Rev. A28, 2195.

Bohr, J., Gibbs, D., Axe, J.D., Moncton, D.E., D'Amico, K.L., Majkrzak, C.F., Kwo, J., Hong, M., Chien, C.L. and Jensen, J. (1989) Physica B159, 93.

Bohr, J. Gibbs, D., and Huang, K, (1990) Phys. Rev. B42, 4322

Burkel, E. (1991) Inelastic Scattering of X-rays with Very High Energy Resolution (Springer-Verlag, Berlin)

Callaway, J. (1991) Quantum Theory of the Solid State, 2nd. Edit. (Academic Press, Boston)

Carra, P., Harmon, B. N., Thole, B. T., Altarelli M. and Sawatzky G. A. (1991) Phys. Rev. Lett. 66, 2495

Collins, S. P., Laundy, D. and Rollanson, A. J. (1992) Phil. Mag. B65, 37

Cooper, M. J. (1985) Rep. Prog. Phys. 48, 415

Cooper, M. J., Collins, S. P., Timms, D. N., Brahmia, A., Kane, P. P., Holt, R. S., and Laundy, D. (1988) Nature 333, 151

Cooper, M. J., Zukowski, E., Collins, S. P., Timms, D. N., Itoh, F., and Sakurai, H. (1992) J. Phys.:Condens. Matter 4, L399

Cowan, R. D. The Theory of Atomic Structure and Spectra (Univ. of California Press, Berkeley, 1981)

Etelăniemi, V., Hämäläinen, K., Manninen, S., and Suortti, P. (1992) J. Phys.:Condens. Matter 4, 879

Friedrich, H, (1991) Theoretical Atomic Physics (Springer-Verlag, Berlin)

Gell-Mann, M. and Goldberger, M.L. (1954) Phys.Rev. 96, 1433.

Gerson, A. R., Halfpenny, P. J., Pizzini, S., Ristic, R., Roberts, K. J., Sheen, D. B., and Sherwood, J. N. (1992) Materials Science and Technology, Vol. 2a Chapter 8

Gibbs, D., Moncton, D. E., D'Aunico, K. L., Bohr, J., and Grier, B. H. (1985) Phys. Rev. Lett. 55, 234 
Gibbs, D., Moncton, D. E., D'Amico, K. L., Bohr, J., and Grier, B. H. (1985) Phys. Rev. Lett. 55, 234

Gibbs, D., Harshman, D. R., Isaacs, E. D., McWhan, D. B., Mills, D and Vettier, C (1988) Phys. Rev. Lett. 61, 1241.

Gibbs, D., Grübel, G., Harshman, D.R., Isaacs, E. D., McWhan, D. B., Mills, D. and Vettier, C. (1991) Phys. Rev. B43, 5663.

Goedkoop, J. B., Thole, B. T., van der Laan, G., Sawatzky, G. A., de Groot, F. M. F., and Fuggle, J. C. (1988) Phys. Rev. B37, 2086

Grotch, H., Kazes, E., Bhatt, G. and Owen, D.A. (1983) Phys.Rev. A27, 243.

Hannon, J. P., Trammell, G. T., Blume, M and Gibbs, D (1988) Phys. Rev. Lett. 61, $1245 ; 62$ 2644(E) (1989).

Hayes, W. and Loudon, R. (1978) Scattering of Light by Crystals (John Wiley \& Sons, New York).

Kinoshita, T. (1990) QED (World Scientific, Singapore).

van der Laan, G., Thole, B. T., Sawatzky, G. A., Goedkoop, J. B., Fuggle, J. C., Esterva, J. M., Karnatak, R., Remeika, J. P. and Dabkowska, H. A. (1986) Phys. Rev. B34, 6529.

Lander, G. H. and Stirling, W. G. (1992) Physica Scripta

Lovesey, S.W. (1986) Condensed Matter Physics: Dynamic Correlations, 2nd. edition (Benjamin/Cummings Publishing, Menlo Park).

Lovesey, S. W. (1987a) J. Phys. C20, 5625

Lovesey, S. W. (1987b) Theory of Neutron Scattering from Condensed Matter Vol. 1 (O.U.P., Oxford)

Margaritondo, G. (1988) Introduction to Synchrotron Radiation (O.U.P., N.Y.)

McMaster, W.H. (1961) Rev.Mod.Phys. 33, 8.

McWhan, D. B., Vettier, C., Isaacs, E. D., Ice, G E., Siddons, D. P., Hastings, J. B.,Peters, C. and Vogt, O. (1990) Phys. Rev. 42, 6007.

Newton, R. G. (1982) Scattering Theory of Waves and Particles (Springer-Verlag, N.Y.)

Platzman, P. M, and Tzoar, N. (1965) Phys. Rev. 139, A410

Ribberfors, R. (1975) B12, 2067: ibid 3136.

Sakurai, J. J. (1987) Advanced Quantum Mechanics (Addison-Wesley Publishing, Redwood City) 
Stirling, W. G. and Lander, G. H. (1993) J. Appl. Phys.

Strange, P., Durham P. J. and Gyorffy B. L. (1991) Phys. Rev. Lett. 67, 3590.

Tang, C. C., Stirling, W. G., Lander, G. H., Gibbs, D., Herzog, W., Carra, P., Mattenberger, K, and Vogt, O., (1992) Phys. Rev. B46, 5287.

Taylor, R.E. (1991) Rev, Mod. Phys. 63, 573.

Thole, B. T., van der Laan G. and Sawatzky G. A. (1985) Phys. Rev. Lett. 55, 2086

Timms, D. N., Brahmia, A., Cooper, M. J., Collins, S. P., Hamouda, S., Laundy, D., Kilbourne, C., and Saint Lager, M-C. (1990) J. Phys.:Condens. Matter 2, 3427

Tolhoek, H.A. (1956) Rev.Mod.Phys. 28, 277.

Vettier, C., McWhan, D. B., Gyorgy, E. M., Kwo, J., Buntschuh, B. M., and Batterman, B. W. (1986) Phys. Rev. Lett. 56, 757

Weissbluth, M. Atoms and Molecules (Academic Press, N.Y. 1978)

Weissbluth, M. (1989) Photon-Atom Interactions (Academic Press, Boston) 
Primary Photon

Target Sample

fluorescent $x$ - rays heat

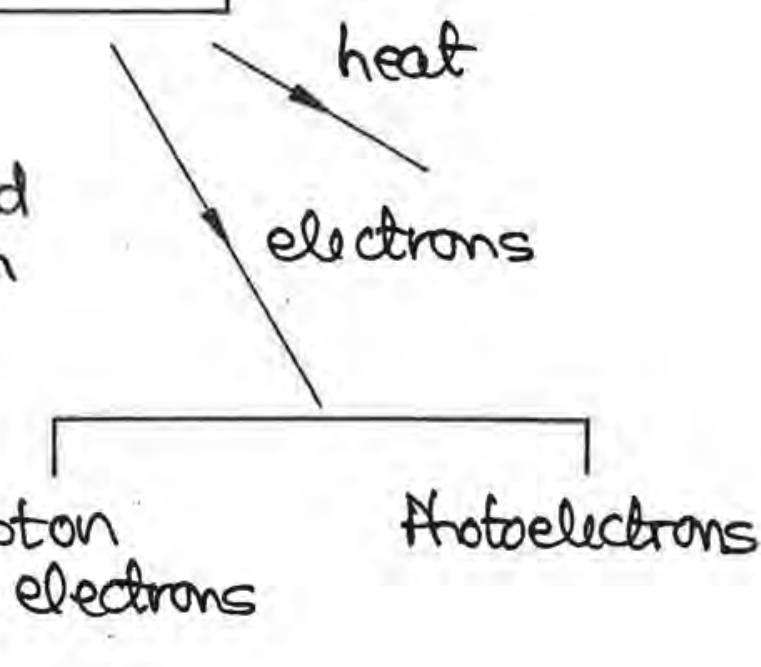

coherent incoherent Compton
(Bragg reflections) (Compton) recoil electrons

$-1-$ 


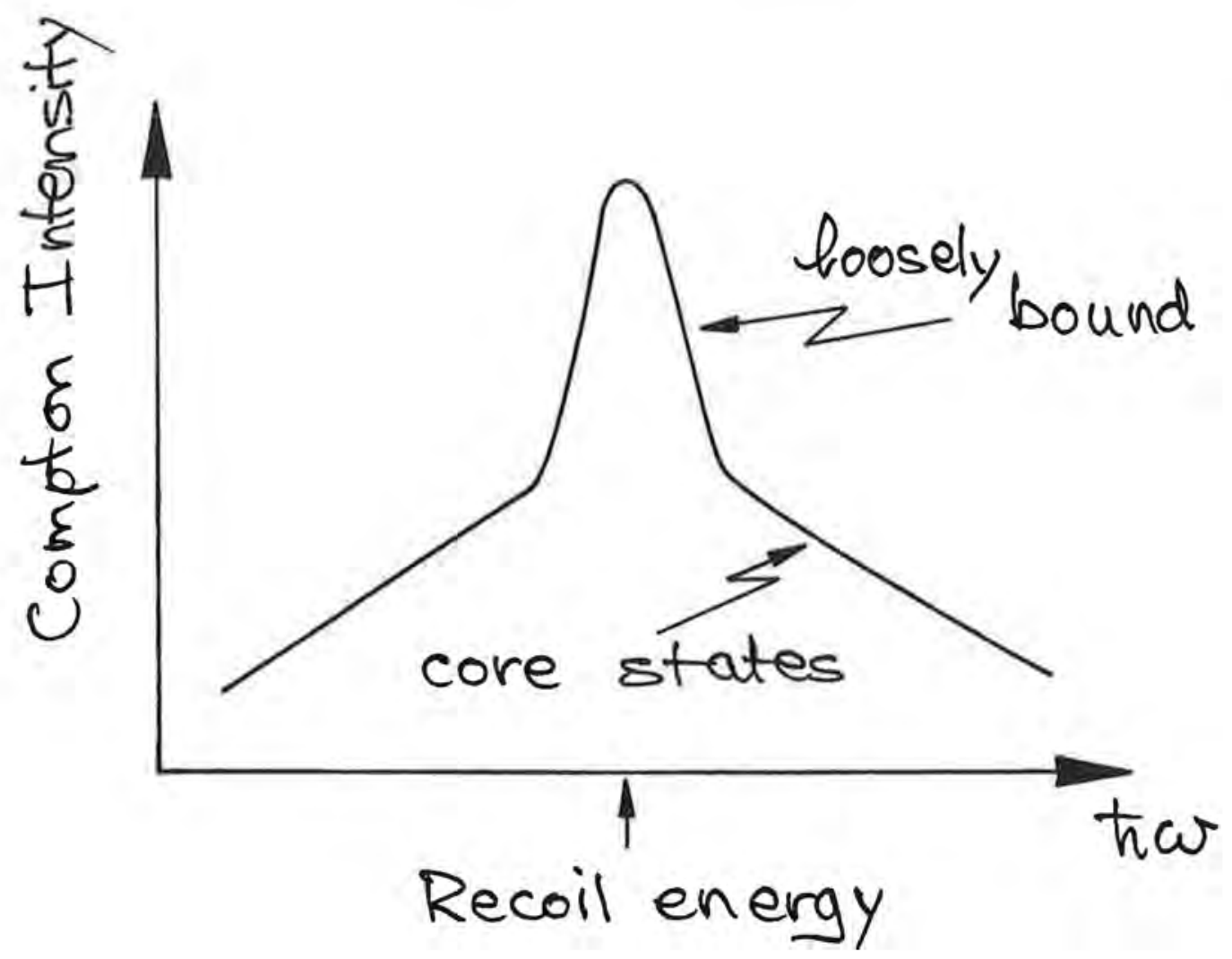




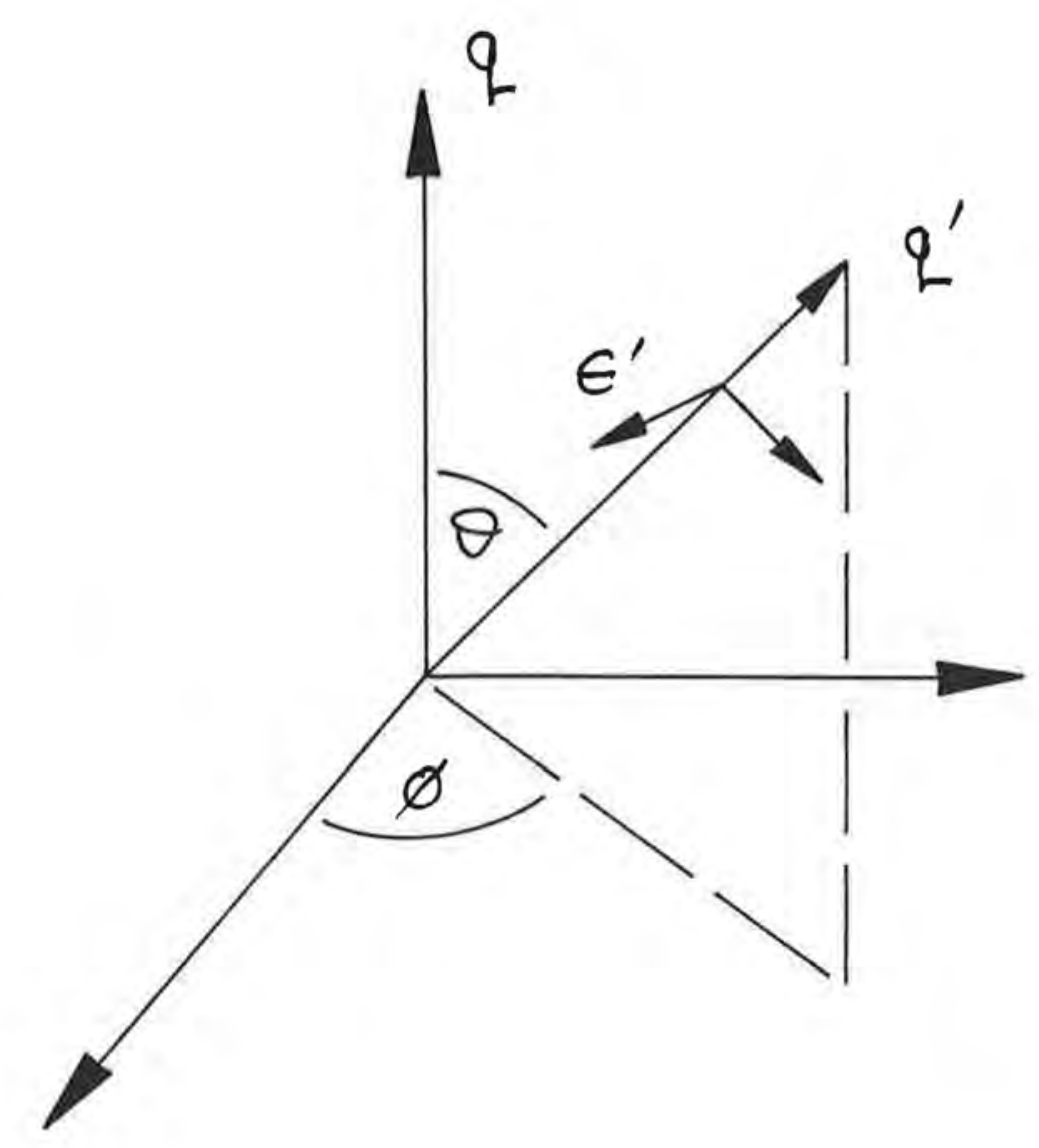

$-3-$ 


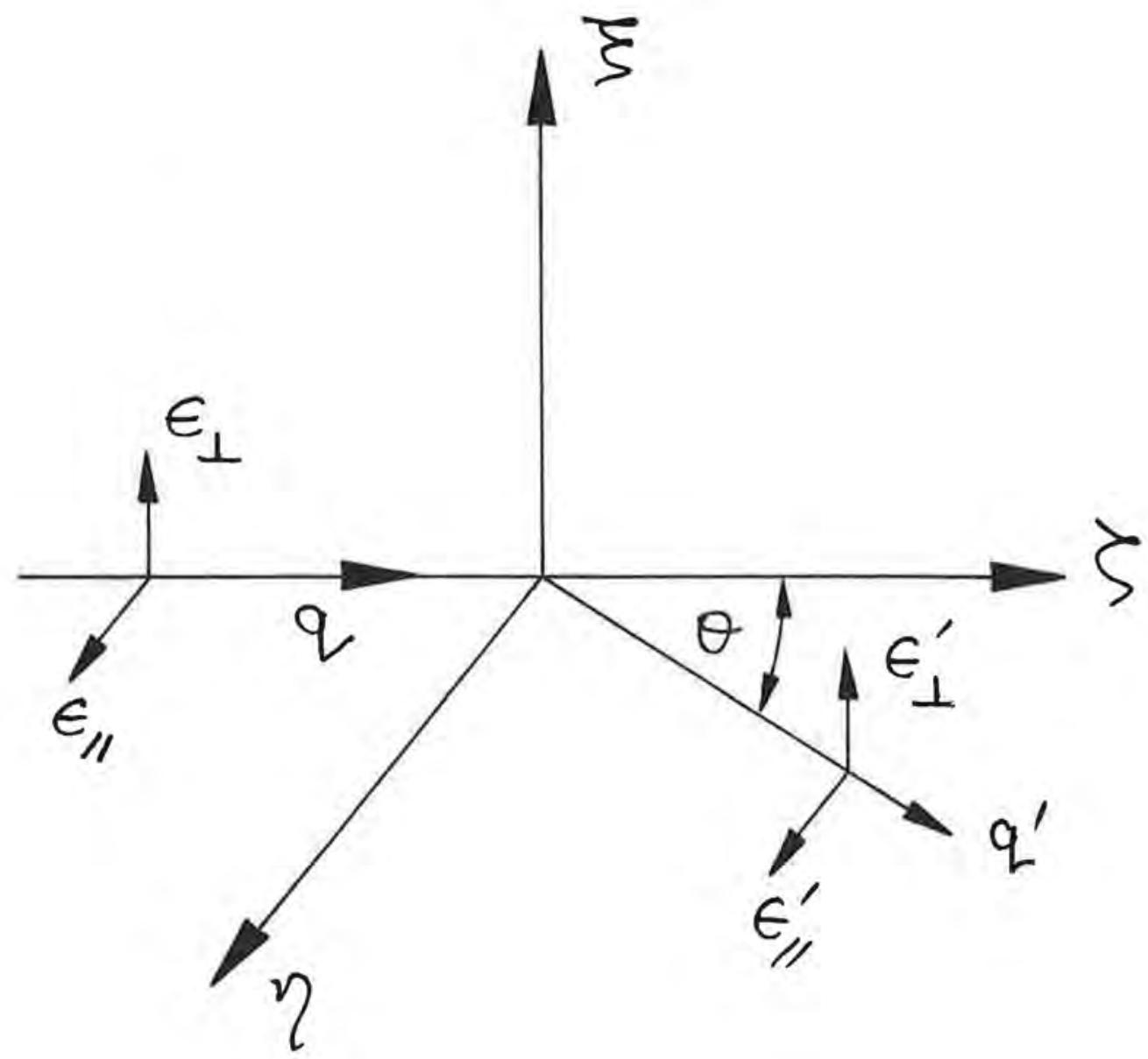

$-4-$ 


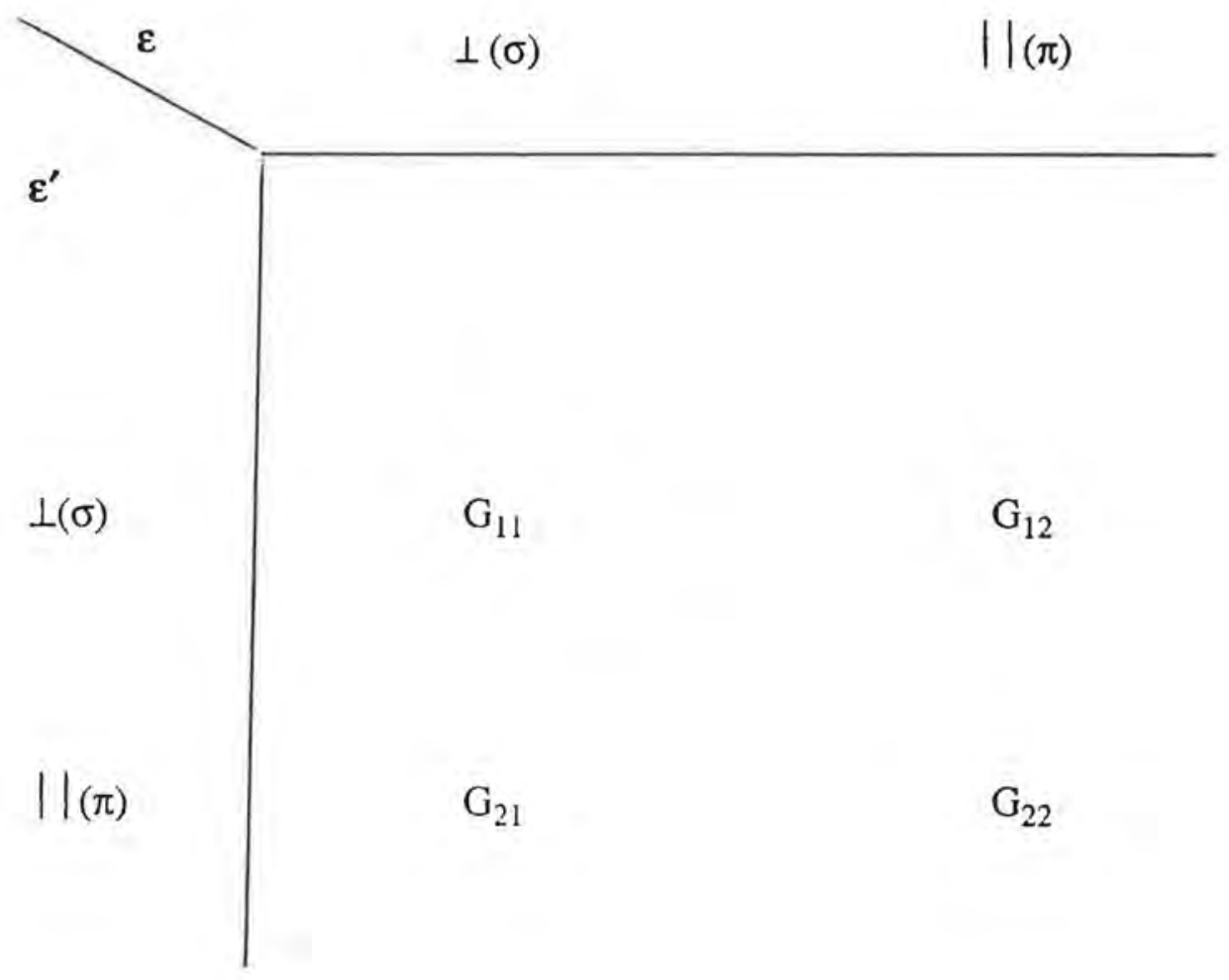

$-5$ 

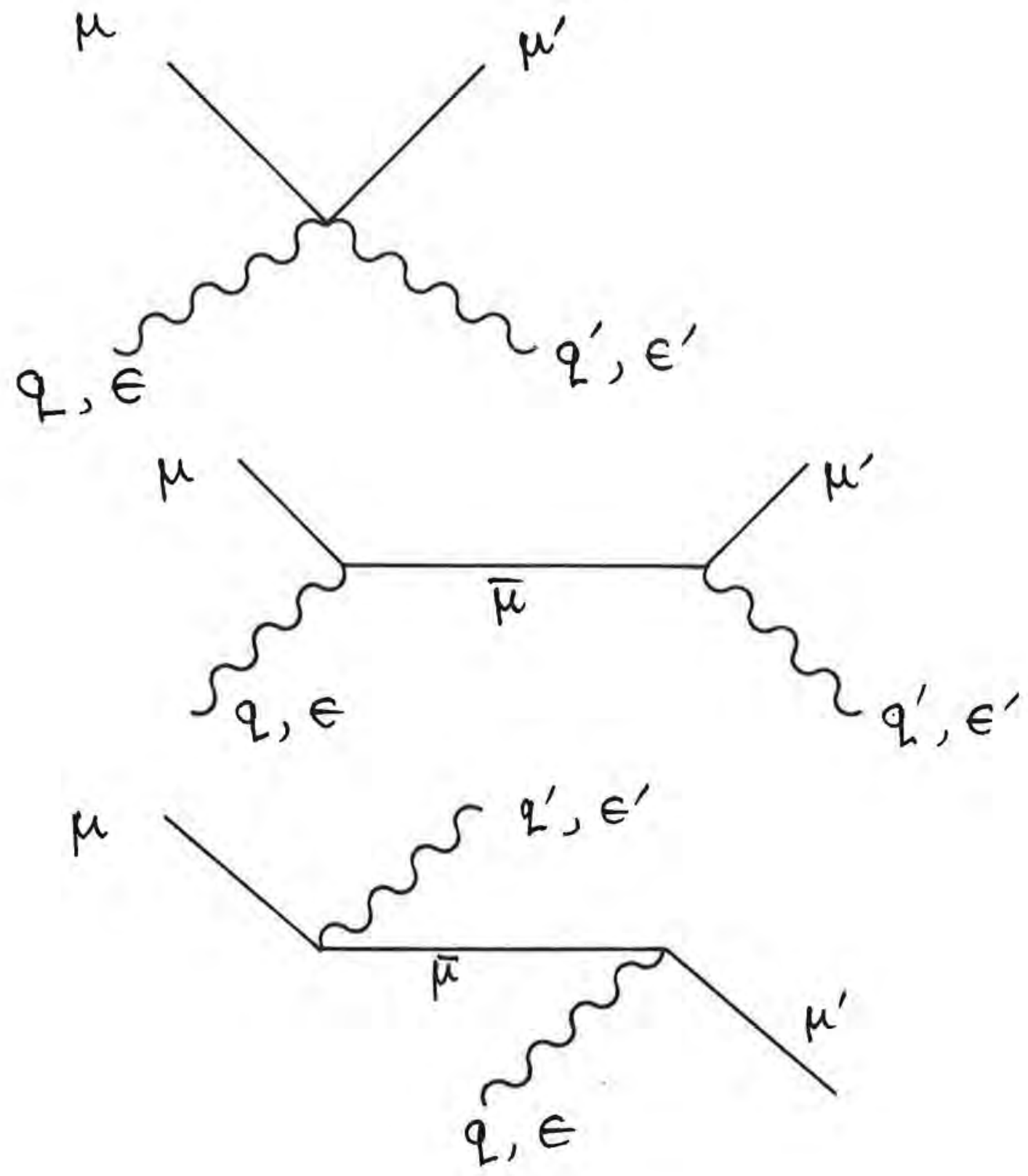


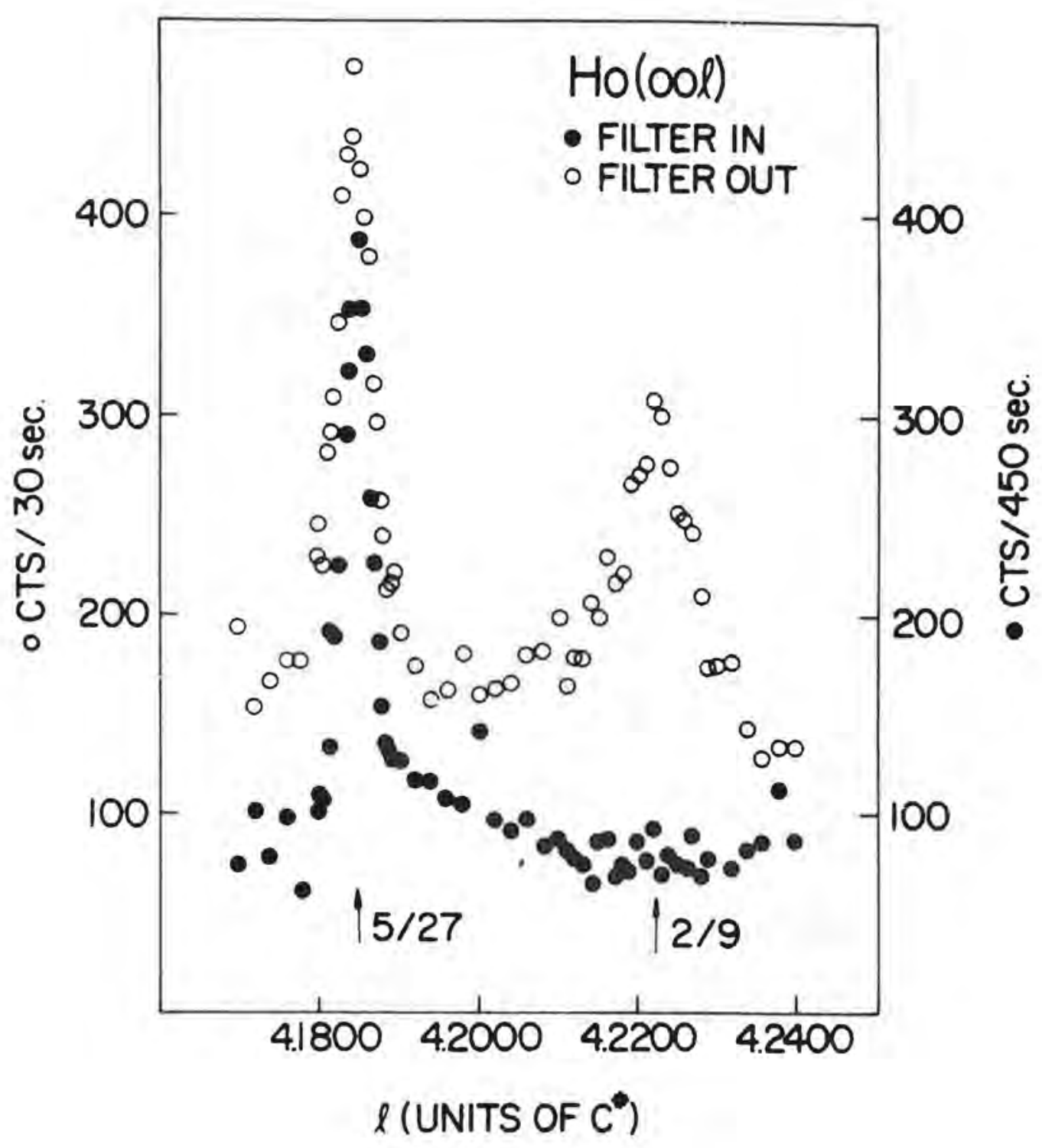


1

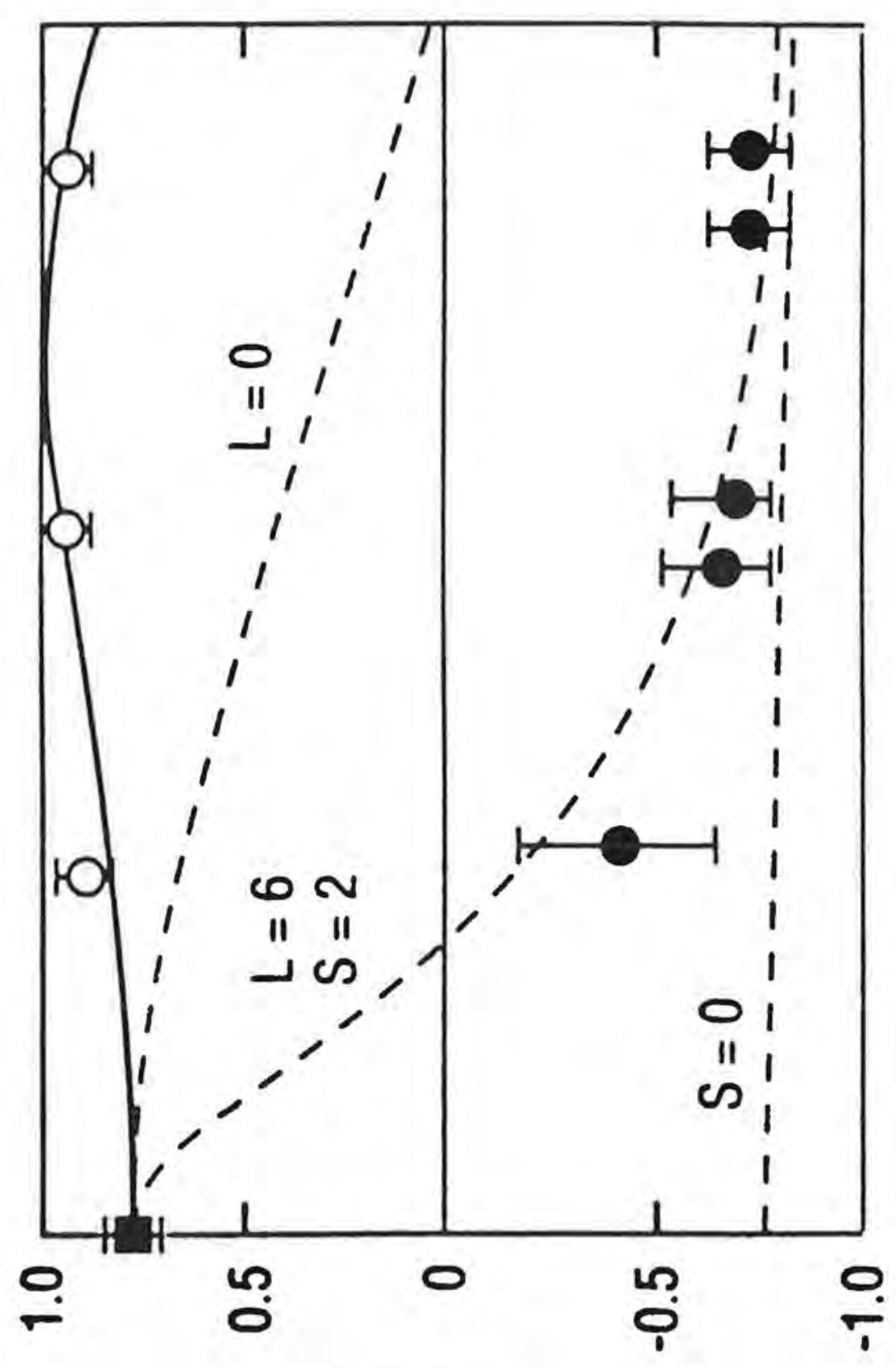

,d NOIIVZIY

Y $\forall$ जNIT J0 $\exists \exists Y 9 \exists 0$ 


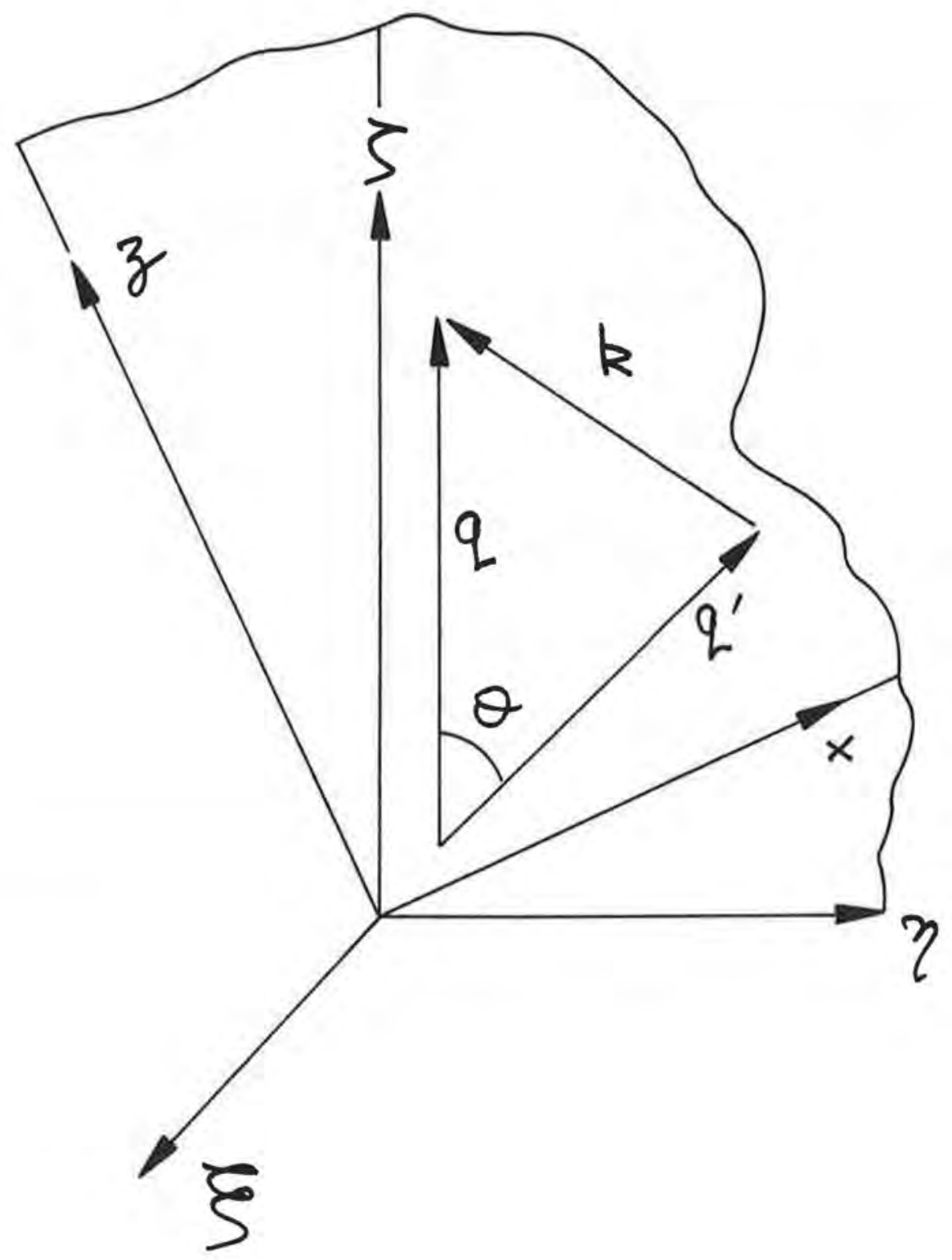

$-9-$ 


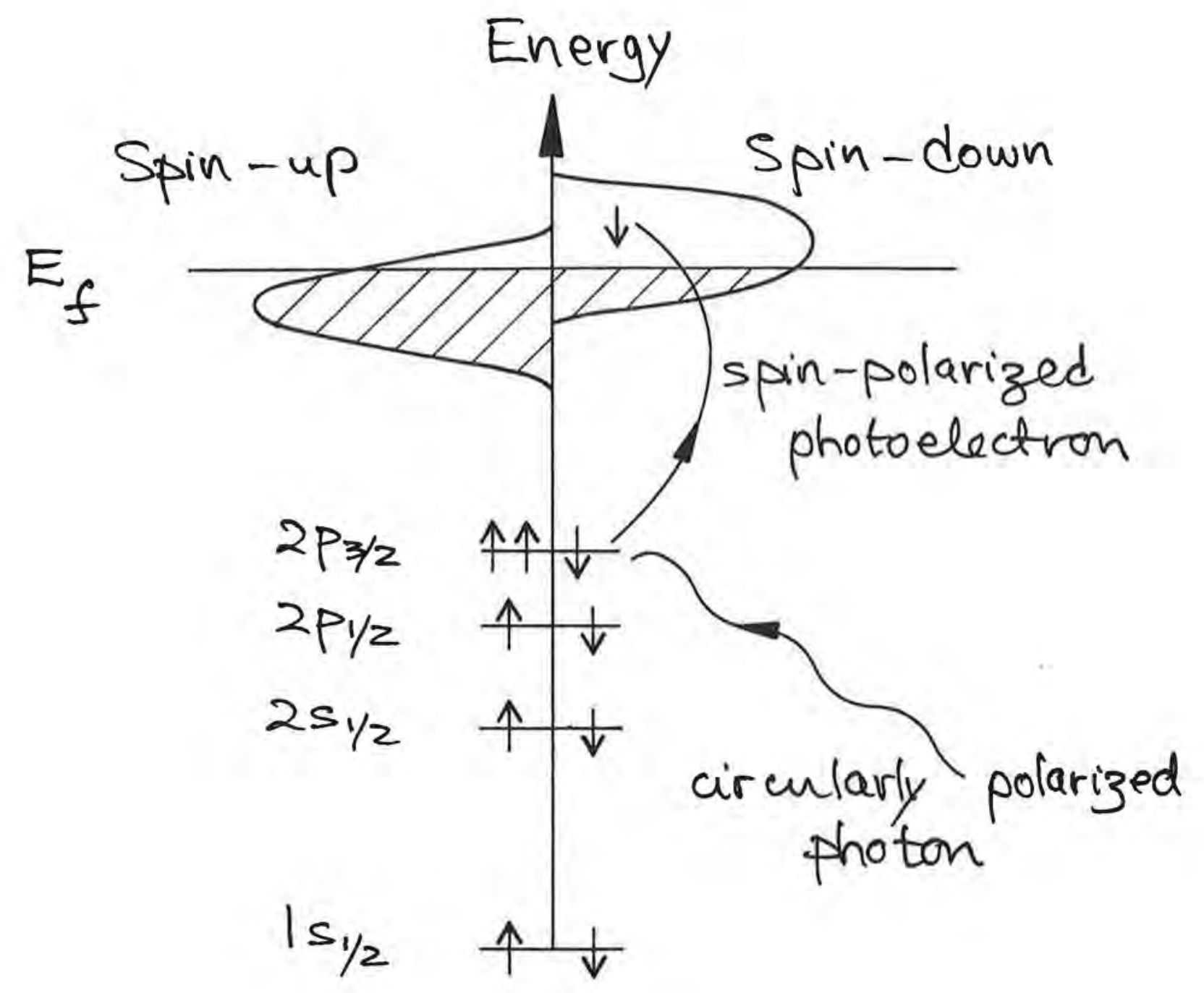

$-10-$ 


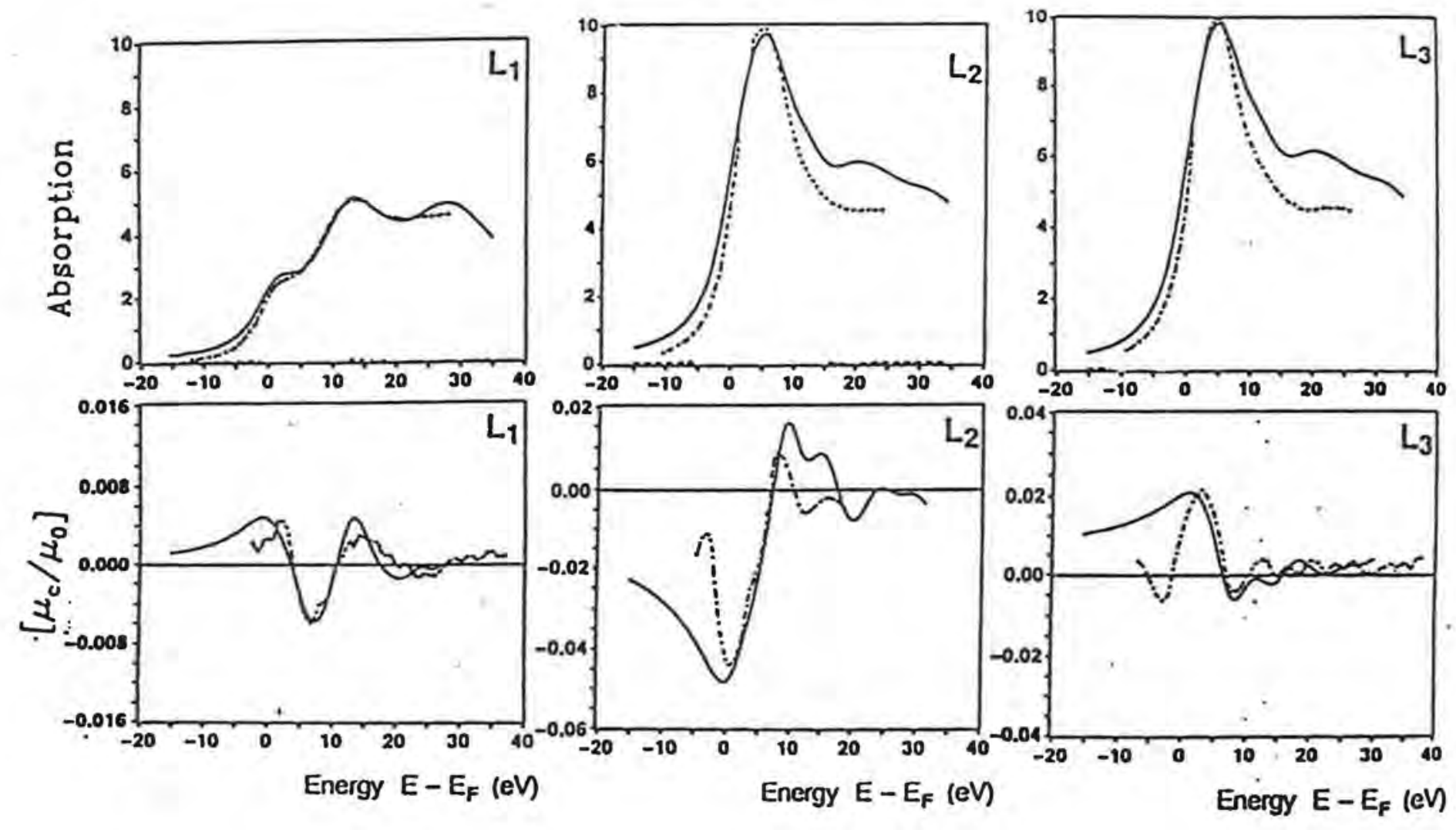


$\frac{1}{1}$

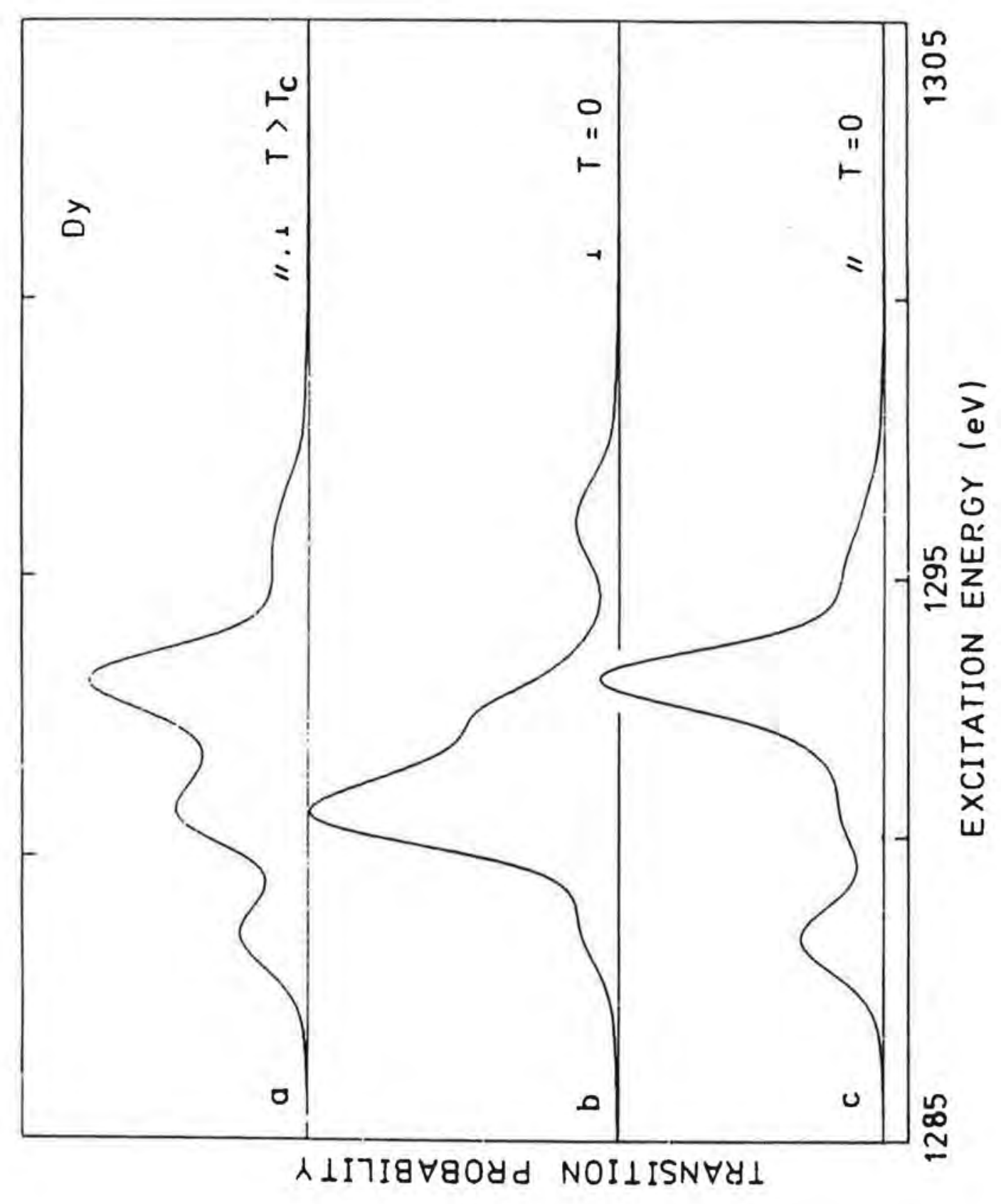




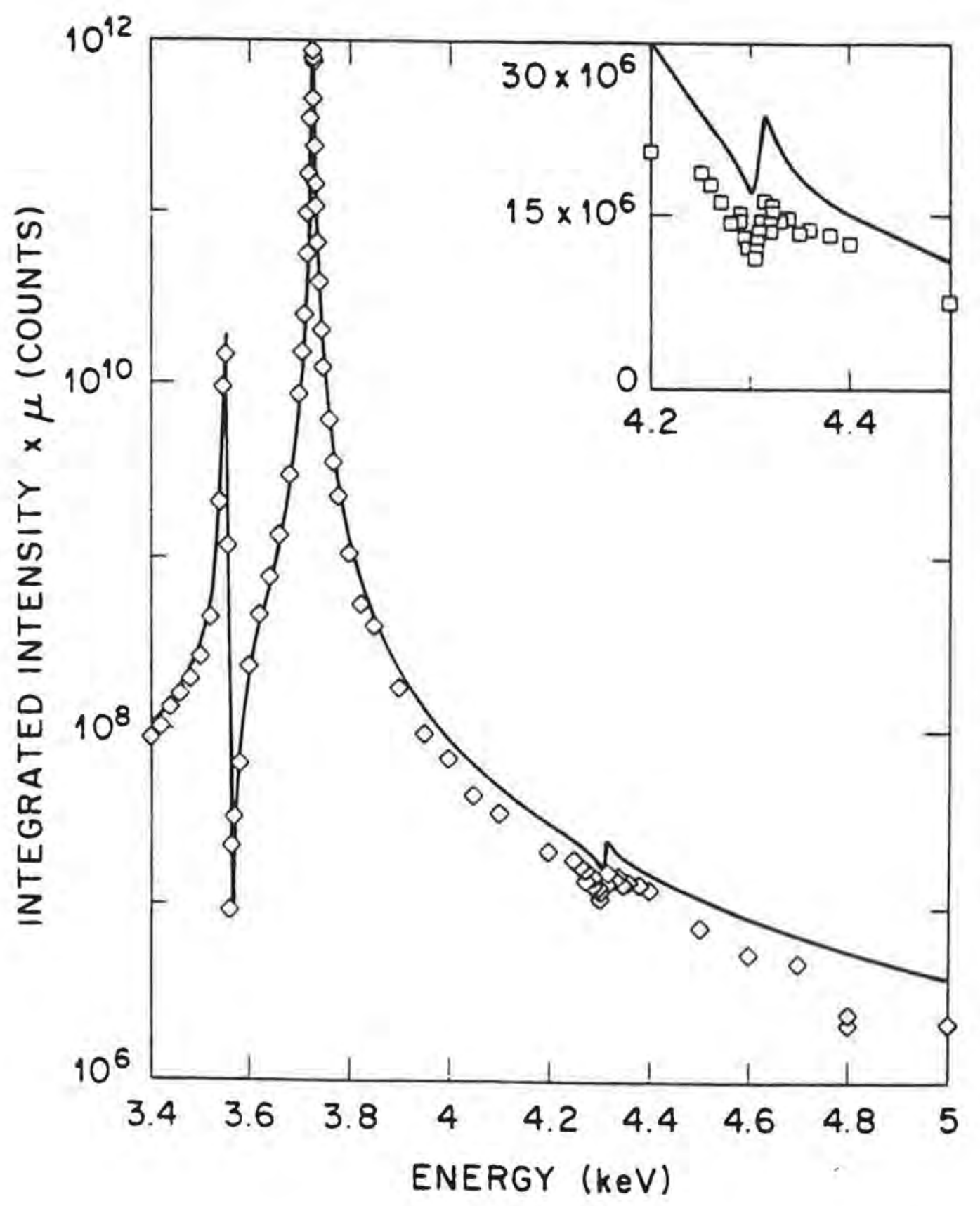

$-13-$ 
Figure (14)
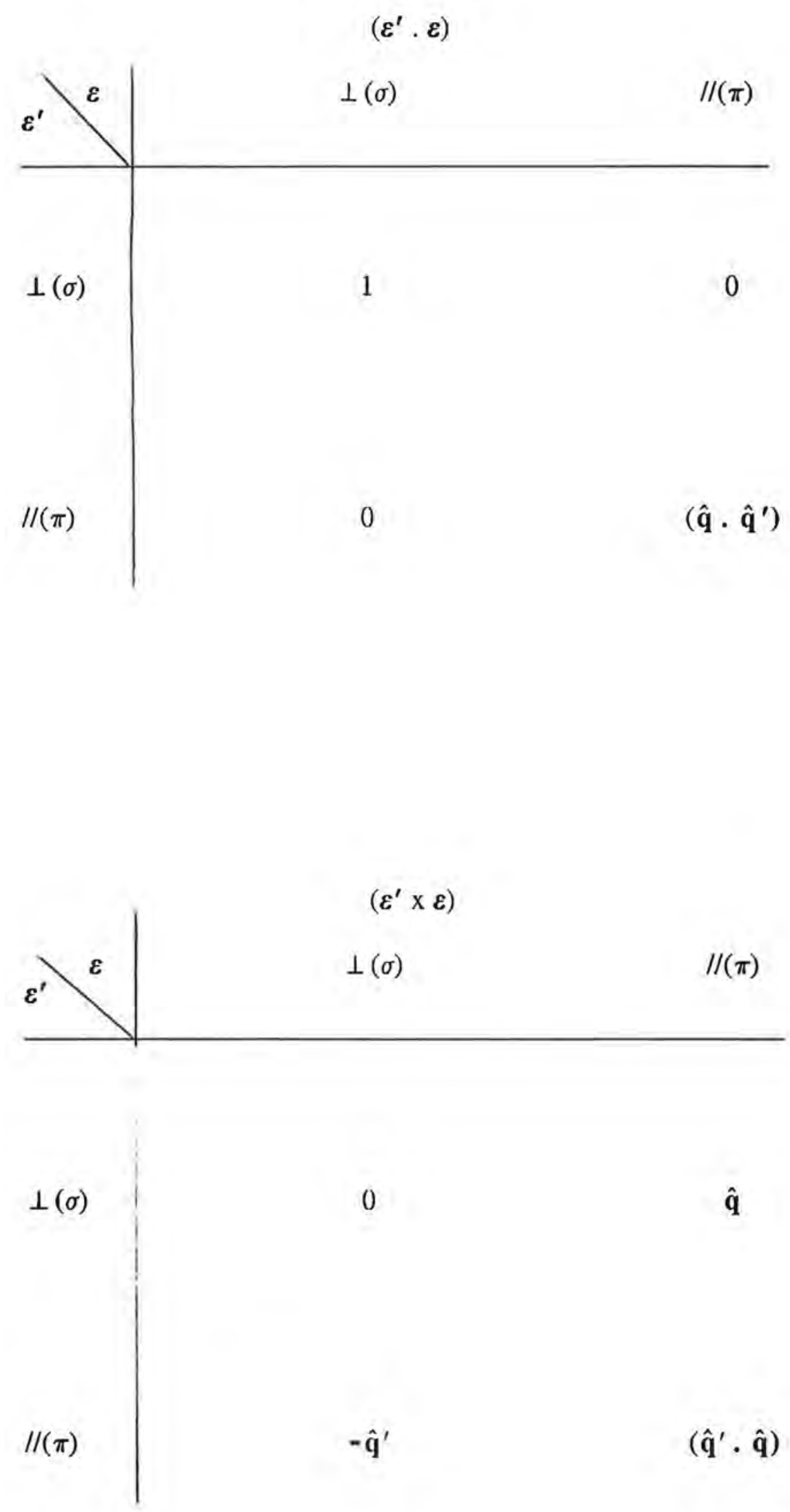


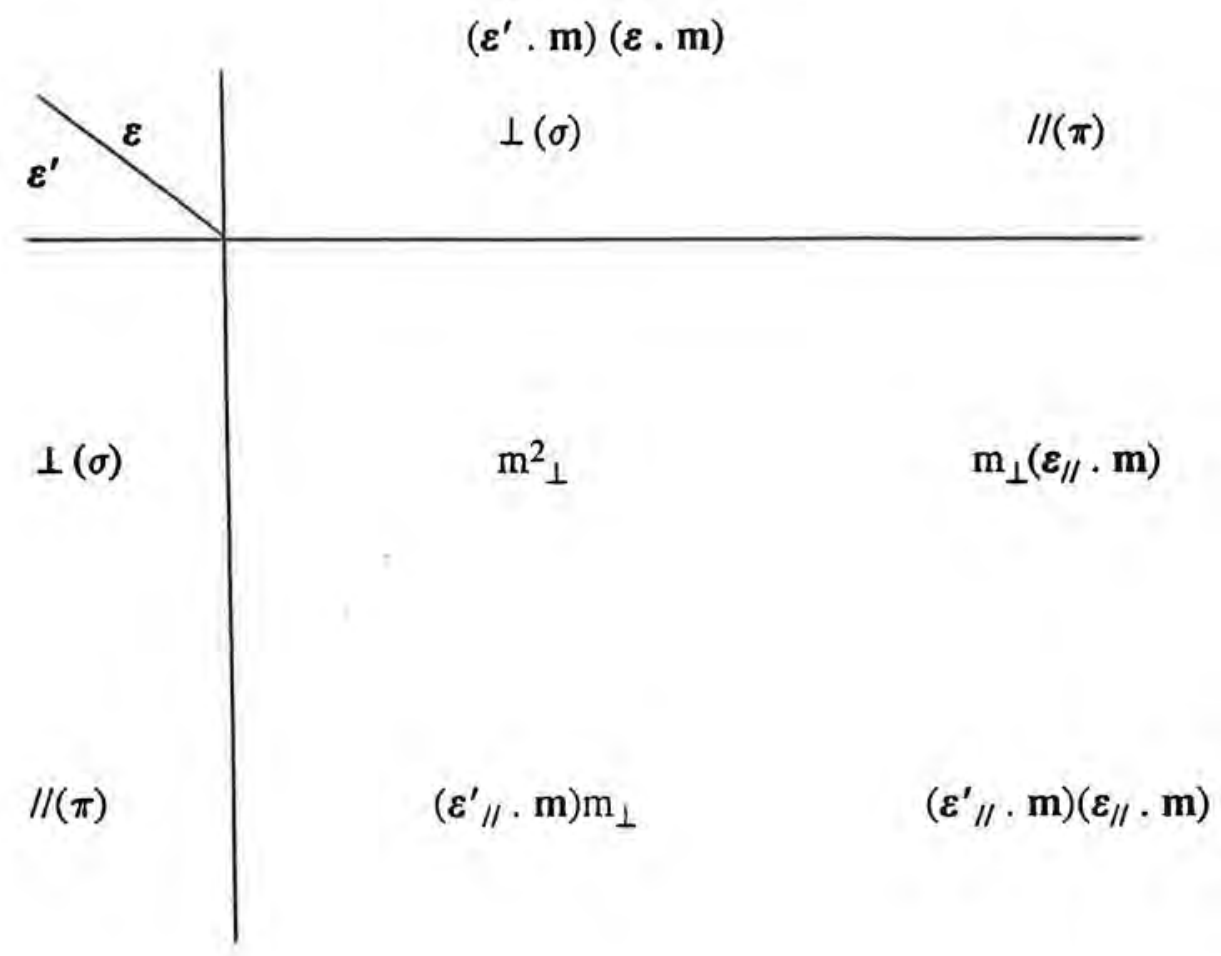




